\title{
On the characteristics and detectability of intracranial microembolic signals
}

Citation for published version (APA):

Mess, W. H. (2003). On the characteristics and detectability of intracranial microembolic signals. [Doctoral Thesis, Maastricht University]. Universiteit Maastricht. https://doi.org/10.26481/dis.20030502wm

Document status and date:

Published: 01/01/2003

DOI:

10.26481/dis.20030502wm

Document Version:

Publisher's PDF, also known as Version of record

\section{Please check the document version of this publication:}

- A submitted manuscript is the version of the article upon submission and before peer-review. There can be important differences between the submitted version and the official published version of record.

People interested in the research are advised to contact the author for the final version of the publication, or visit the DOI to the publisher's website.

- The final author version and the galley proof are versions of the publication after peer review.

- The final published version features the final layout of the paper including the volume, issue and page numbers.

Link to publication

\footnotetext{
General rights rights.

- You may freely distribute the URL identifying the publication in the public portal. please follow below link for the End User Agreement:

www.umlib.nl/taverne-license

Take down policy

If you believe that this document breaches copyright please contact us at:

repository@maastrichtuniversity.nl

providing details and we will investigate your claim.
}

Copyright and moral rights for the publications made accessible in the public portal are retained by the authors and/or other copyright owners and it is a condition of accessing publications that users recognise and abide by the legal requirements associated with these

- Users may download and print one copy of any publication from the public portal for the purpose of private study or research.

- You may not further distribute the material or use it for any profit-making activity or commercial gain

If the publication is distributed under the terms of Article $25 \mathrm{fa}$ of the Dutch Copyright Act, indicated by the "Taverne" license above, 


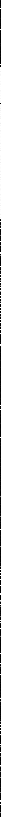




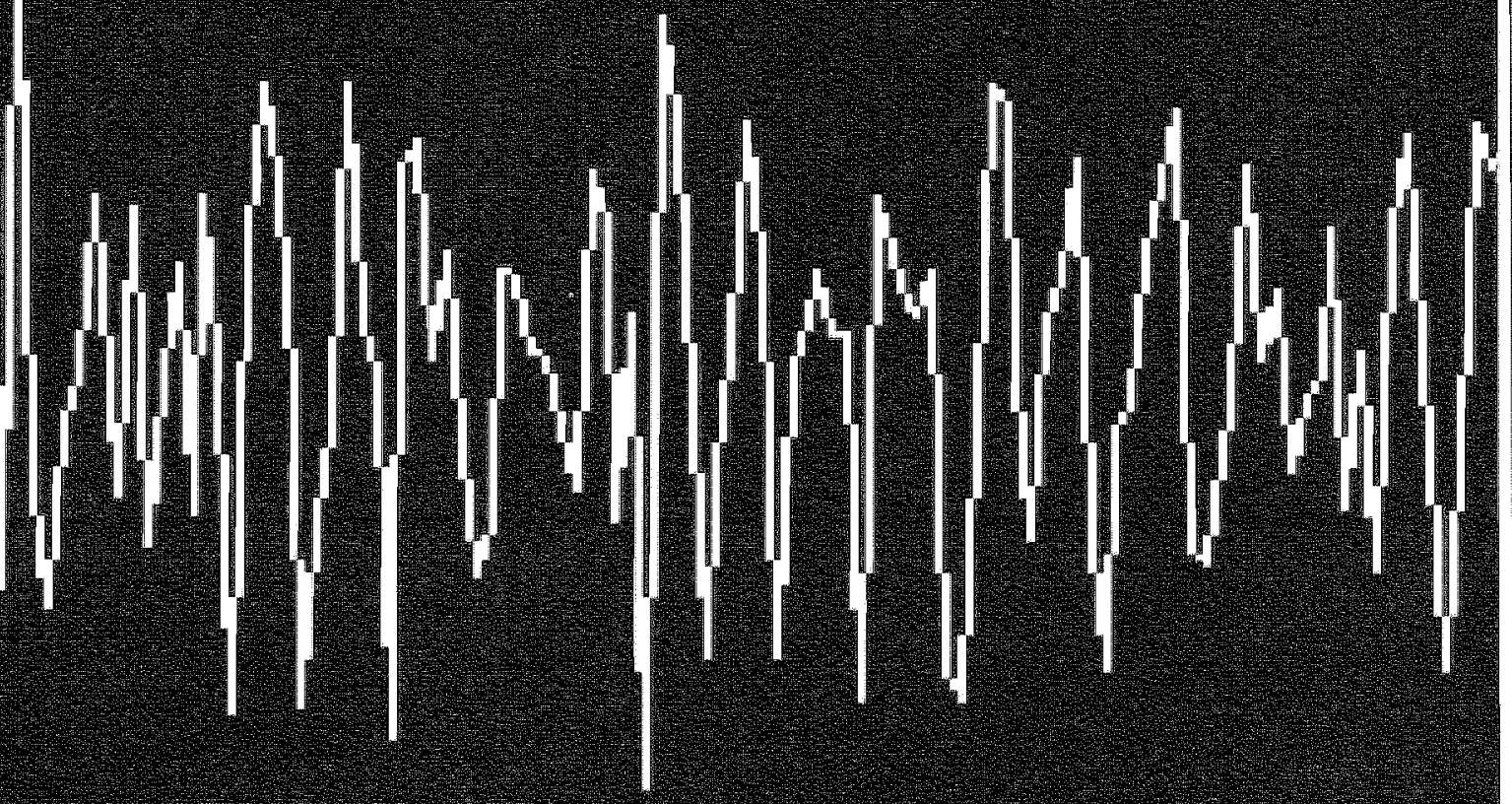




\section{On the characteristics and detectability of intracranial microembolic signals}

W.H. Mess 
On the characteristics and detectability of intracranial microembolic signals 
On the characteristics and detectability of intracranial microembolic signals

\section{PROEFSCHRIFT}

ter verkrijging van de graad van doctor aan

de Universiteit Maastricht,

op gezag van de Rector Magnificus

Prof. dr. A.C. Nieuwenhuijzen Kruseman,

volgens het besluit van het College van Decanen

in het openbaar te verdedigen

op vrijdag 2 mei 2003 om 14.00 uur

door

Wermer Heinrich Mess

geboren op 7 februari 1961 te Recke in Westfalen (D) 
Promotores

Prof, dr. ir. A.P.G. Hoeks

Proff, dr. D.H. Ewans (University of Leicester; UK)

Copromotor

Dr.R.G.A.Ackerstaff (Antonilus Ziekenhuis Nieuwegein)

Beoordelingscommissie

Prof. dr. P.J.E.H.M. Kitslaar (voorzitter)

Prof.dr. ir. T. Artts

Prof. dr. M.G. Hennerici (Universität Heidelberg: D)

Dr. J. Loddler

Prof.dr. B. Mochtar 
Layout and cover illustration

Fabrice Hermans, Nuth BNO

On the characteristics and detectability of intracranial microembolic signals.

W.H. Mess

Thesis University Maastricht

ISBN 90-9016790-0

(c) 2003, W.H. Mess, Maastricht, The Netherlands. 


\section{Contents}

1 Introduction

2 Automatic embolus detection compared with human experts;

a Doppler ultrasound study

3 Middle cerebral artery anatomy and characteristics of embolic signals: a dual gate computer simulation study

4 A new algorithim for off-line automated emboli detection based on the pseudo Wigner power distribution and the dual gate TCD technique

5 Microembolic sïgnal description: a reappraisal based on a customized digital post-processing system

6 The depth of microembolic signal direction change corresponds with vessel anatomy

7 Epilogue

8 Appendix

Summary

Samenvatting

Zusammenfassung

Dankwoord

Publications

Curriculum Vitae 
introcueton 


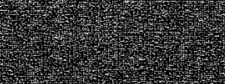

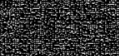

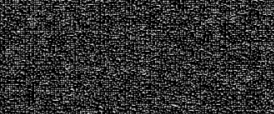

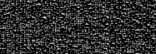

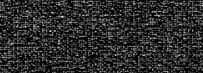

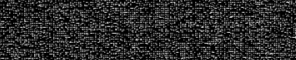

药

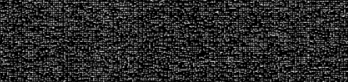

(1.

\begin{tabular}{|c|c|c|c|}
\hline \\
\hline
\end{tabular}

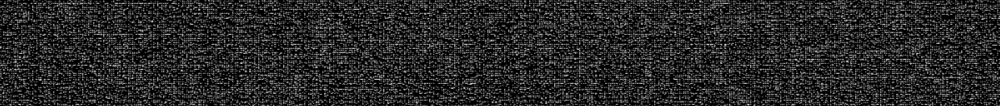

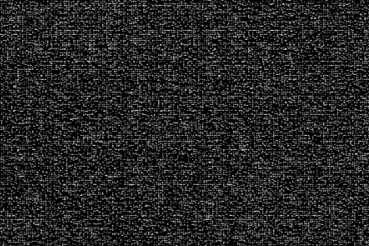

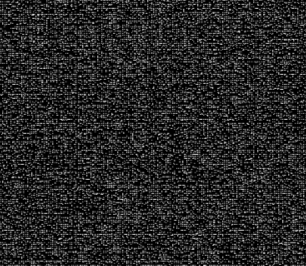

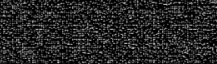

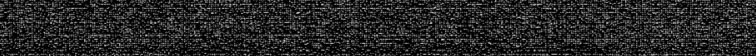

1.

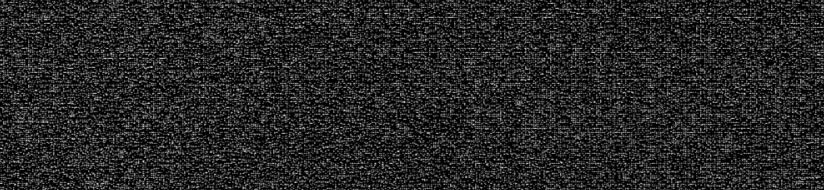

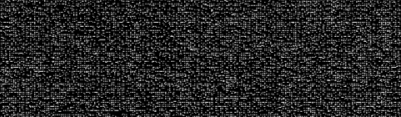

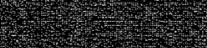

(1)

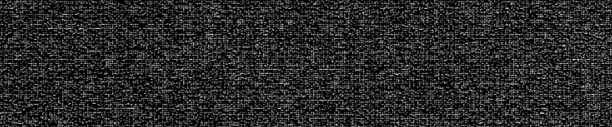


Stroke is the third leading cause of death in most western countries and is responsible for the possibly even more important burden of short-term and prolonged disability (Warlow et al. 2001). In the majarity of patients a stroke is caused by a drop of blood supply to a given area of brain tissue below a critical threshold, which results in irreversible neuronal damage. Atherosclerosis, especially of the carotid arteries in the neck, often can be identified as the pathophysiological mechanism leading to stroke. For a better understanding, first the anatomy of cerebral blood supply is rewiewed briefly (Gray 1918), second, the pathophysiological relationship between atherosclerosis and stroke will be discussed, and third, the focus will be on ultrasound and its. application in this field of interest. Finally, the introduction will outline the main objectives of the thesis.

\section{Blaod supply of the brain: vascular anatomy}

Several centimeters distally from the origim of the aorta, the innominate artery branches off on the right side of the neck (Fig. I). This vessel splits after several centimeters into the right subclavian artery and the right common carotid artery (CCA). On the left side the CCA and the subcliavian artery originate separately from the aorta. A vertebral artery originates from each subclavian artery and travels cranially in the posterior part of the neck in wery close relationship to the cervical vertebrae (Fig. 2). Both CCAs also travel cranially. Approximately at the level of the larynx the vessel diameter becomes wider, forming the carotid bulb, from which the internal and external carotid artery (ICA and ECA) emerge. The latter soon gives rise to several branches supplying the skull, the muscles, the glands and the skin of the head and neck with blood. The ICA runs cranially without giving off any branch before entering the skull.

Intracranially, the ICA gives rise to the ophthalmic artery and then, on the floor of the skull base, 5 plits into the middle cerebral artery (MCA), which travels laterally, and the anterior cerebral artery (ACA), which first runs medially over a very short segment and then ascends between the two hemispheres (Fig. 3 a and b). Usually, a connection between both ACAs, just before the vessel ascends, is present; the anterior communicating artery (AcomA). The AcomA enables blood flow from one side to the other. This phenomenon is called collateralisation and can play an important rolle in the case of a severely stenosed or occluded carotid antery an one side of the neck.

The vertebrall arteries enter the skull posteriorly in the vicinity of the spinal cord. At the level of the brain stem they merge and give rise to the basilar artery, which after several centimeters splits into a left and right posterior cerebral artery (PCA). The PCA travels laterally in a curved course. At the most rostral part of it, the posterior communicating arteries (PComA) connect the PCAs on either side with the very distal part of the ICA or the proximal MCA. So, on the floor of the skull base, a circle of vessels is formed, which enables different ways of collateralisation. However, this circle of Wilis (named after the man who first described the anatomy and physiology in detail) very often is incomplete (Lang et al. 1979). Consequently, the possibilities for intracranial collateralisation can be limited in an individual patient to a variable degree. 
Theoretically, a pathological process anywhere in wessels supplying the brain with blood could cause a stroke, but from a dinical point of view, atherosclerosis at the bifurcation of the CCA into the ECA and ICA (Caplan 1993) clearly is the most important pathophysiological mechanism for stroke.

\section{Atherasclerosis and its relation to stroke}

Atherosclerosis now is regarded as a chronic inflammatory process (Ross 1999), that starts very" early in life. Genetic factors as well as environmental aspects (e.g. smoking) interact with the interplay between blood constituents, like lymphocytes or low density lipoprotein (LDL), and the vessel wall, especially the inner layer, the endothelium. Moreover, infection with chlamydia pneumoniae seems also to be involved in plaque formation, probably mainly due to interaction with platelets (Vainas et al.2002). The impact of the genetic factors is currently thought to be responsible for the individual susceptibility to develop a clinically relevant atherosclerotic lesion. The complex process of atherosclerosis, in first instance, leads to a thickening of the arterial wall. However, a concomitant dilation takes place, so that no lumen narrowing occurs. If the process continues, the atherosclerotic lesion protrudes into the lumen and forms a plaque, which finally forms a so-called "complicated lesion" with a core of necrotic and lipid tissue, covered by a fibrous cap. In the final stage, rupture of the fibrous cap may occur, which favors the deposition of platelets; even complete thrombosis of the vessel often accompanies these complicated lesions. Hemodynamics are commonly thought to be responsible for the rather focal atheroscleratic affection of the extracranial arteries. The blood flow pattern in the carotid bulb depends on the specific anatomical situation (Bergman et al. 2001). A zone of relatively low flow velocities and even reverse flow commonly exists at the lateral side of the origin of the internal carotid artery, opposite to the origin of the external carotid artery. The ensuing low "shear stress" has found to be a promotor for the formation of an increased initima-media thickness (Gnasso et al. 1996). Yet, it is not known, what the direct pathophysiologic link between this observation and the development of atherosclerotic plaques is.

In terms of stroke, atherosclerotic lesions of the internal carotid artery can disturb the blood supply of the brain in two ways. First, if the plaque grows and facultatively in conjunction with additional thrombosis obstructs more than $80 \%$ of the lumen, a so-called "hemodynamically relevant stenosis" or even a complete occlusion emerges. This obstruction has as a consequence that less blood per time is delivered to the brain (Flanigan et al. 1977). In the case of poor collateralisation (Derdeym et all. 1999), a stroke then can be the clinical consequence, especially if the blood pressure temporarily is low, as may be the case under a variety of circumstances (Warlow 2001). Second, embolic material can be teared off the plaque and obstruct intracranial vessels with as a result a stroke. Especially, thrombotic material is likely to form emboli, but in patients with carotid artery plaques, other material such as cholesterol crystals can emerge trom the atherosclerotic lesion (Caplain 1993). Both, the hemodynamic aspect and the embolic aspect of plarque formation at the 


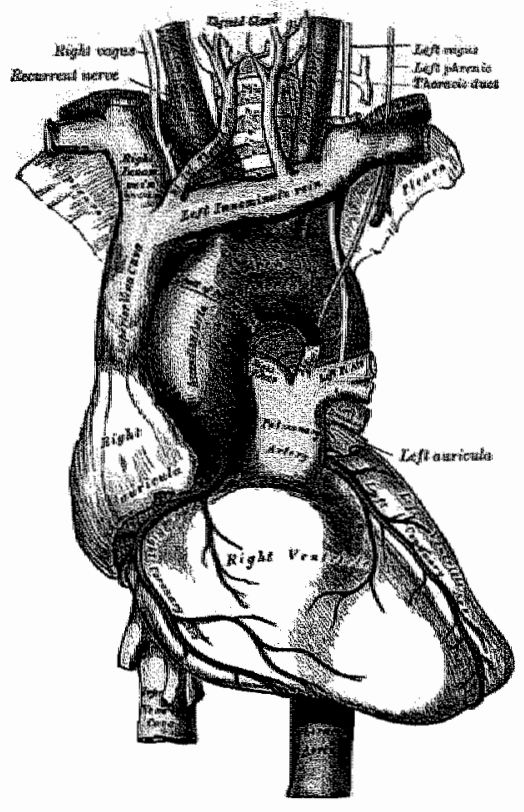

Figure 1

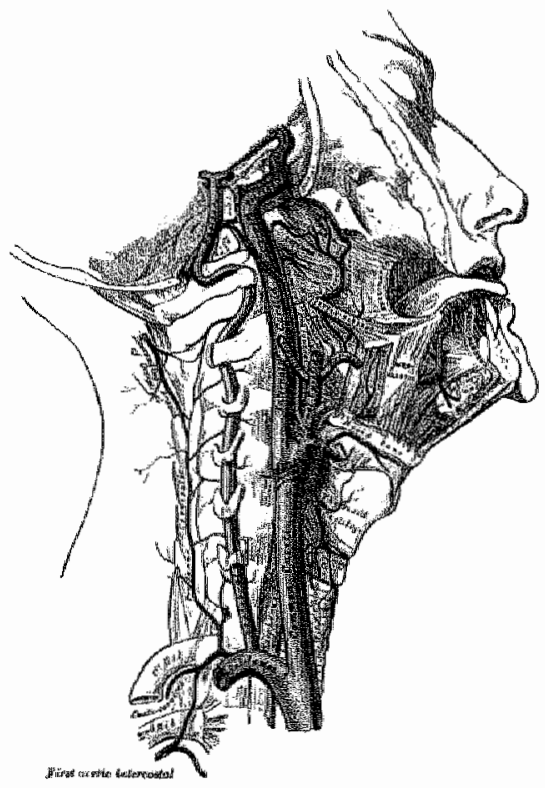

\section{Figure 1}

The andomical drawing shows the heart, the aortic arch and the major arteries branching off cramially. These are the innominate artery, which later gives rise to the right subclawan artery and the right common carotid artery, the left common carotid artery and the left subrlavian artery.

ffigure ils taken from: Gray 19 19)
Figure 2

On the pight side of the neck, the course of the common carotid artery and the vertebial artery is shown At the liewel of the larym, the common carotid artery splits into the extrermat and internal carotid artery.

(figume is taken from:Gray 197:84 
Carotid bifuration contribute to a substantial degree to stroke, both aspects might even interact pathophysiologically (Caplan and Hennerici 1998).

If an atherosclerotic lesion is diagnosed in a patient, who is asymptomatic for that lesion, no therapeutic consequences will be drawn (Warlow et al. 2001). If a stroke or a short lasting ischemic event ( 24 hours; transient ischemic attack, "TIA") has occurred in a territory of the brain that is depending on a carotid artery with an atherosclerotic lesion, the latter is regarded as a symptomatic lesion. Two principal therapeutic possibilities with regard to the carotid artery plaque exist: First, the lesion can be surgically removed by means of a carotid endarterectomy (CEA) and second, platetellet aggregation inhibitors (e.g. aspirine) can be prescribed. Both regimens are aimed at preventing the patient from a next stroke or TIA ("secondary prevention"). Two large-scalle studies (Barnett et al. 1998; Rothwell et al. 1999) compared prospectively two patients groups with a hemodynamically significant stenosis, whereby one group was operated on and both groups received anti-platelet medication. The CEA group had a better prognosis, but the difference was relatively small. It was calculated that 13 patients had to be treated surgically to prevent one stroke, but only if the perioperative complication rate is kept wery low (2-3\% stroke or death).

So, ironically the surgical procedure intended to prevent the patient from a stroke bears also the risk of causing a stroke. Owing to this, CEA has to be performed as safe as possible. Besides the skill of the surgeon (Pearce et al. 1999) it is reasonable to assume that information on brain function and cerebral blood flow during the procedure will contribute to the outcome, provided that it is known what can be expected as normal and that it is possible to interfere if pathological deviations occur.

The brain function itself can be monitored intraoperatively by means of an electroencephalogram (EEG) or somatosensory evoked potentials (SSEP; Graybeal 1995). Continuous non-imvasive monitoring of the blood flow in the MCA can only be accomplished by the use of transcranial Doppler (TCD) sonography (Naylor 2001), which is a specialised application of ultrasound. Therefore, a short introduction of ultrasound and its specific employment in patients with carotid atherosclerosis will be given.

\section{Ultrasound, TCD and emboli detection}

The "Doppler effect" first described in the $19^{\text {th }}$ century, allows for detecting motion between an observer and a wave source by means of measuring the frequency shift between them (Doppler 1842, Buys Ballot 1845). It lasted a hundred years, before Doppler ultrasound was introduced into medicine for the study of blood flow in peripheral arteries (Evans and McDicken 2000). Already in 1965, an attempt was made to evaluate the intracranial arteries by means of ultrasound (Freund 1965). However, the measurement of blood flow velocity in the large vessels branching off the circle of Willis became feasible only when Aaslid introduced in 1982 transcranial Doppler sonography (TCD) based on a pulsed Doppler system with a relatively low insonation frequency (Aaslid et al. 1982). 


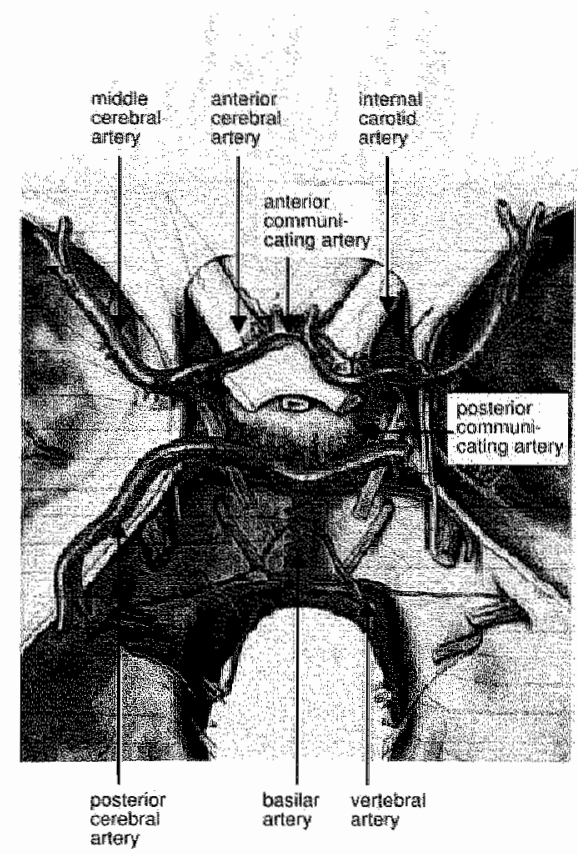

Figure 3a

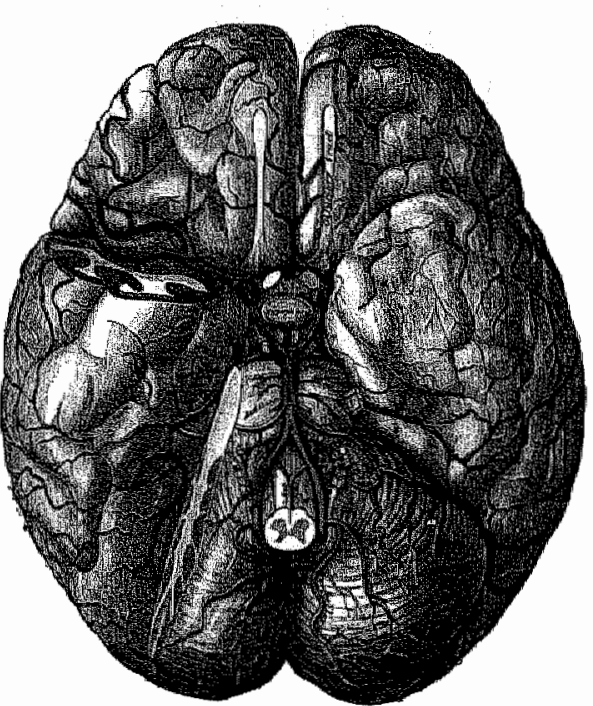

\section{Filgure $3 \mathbf{a}$ and $\mathbf{b}$}

The basal cerebul arteries and their relationship to the skuli (a) and brain (b). Figure $3 a$ shows the most distal part of the intemill carotid artery reaching the base of the skull faterally from the optic chiasm froph. It is evictent from this amatomical reproduction that the middle cerebral artery is the main branch of the internal carotid artery, whist the anterion cerebral antery is a relatively thin vessed originating at the transition from the internal carotid artery sinto the nniddle cerebral artery. Wote the opposite flow drection in thw most distal part of the internal carotid artery compared to the most proximal part of the anterior cerebrall artery. The antefior cerebral artery in its further course lies above the optic nerwe fust before entering the optic chiasm. Near the midline the anterior cerebral artery tums anterion, the left and right artery being consected through a short vessel segrnent, the antealor communicatting artery. The two vertebral arteries enter the skull wis the foramen magnum (bottom), and supply the two posterior cercibral arteries via a short common segment, the basilar artery. Figure 3 b illustrates the relatianship of the circle of witis and the hypophysis, as well as the further ramification of the antertor, middle, and posterior cerebral arteries,

(figure 3a is taken from: Lang et al. 1979, figure 3b is taken from: Gray 1918) 
In most human beings, the temporal bone has a relatively thin area compared to the other skull bones. Ultrasound cam penetrate the skull wia this transtemporal bone window. Depending on the position of the transducer emitting the ultrasound signal and the depth from which the ultrasound reflections are arialyzed, the thiree large basal cerebral arteries can be distinguished. A further discussion of the physics of ultrasound and technical aspects of Doppler systems is given in the appendix.

Today, TCD has found wide acceptance as a tool for evaluation of cerebral hemodynamics in a variety of clinicall settings, e.g. monitoring during carotid endarterectomy or the evalluation of the collateralisation pathways in patients with extracranial arterial disease. An unexpected use of TCD was reported by Spencer in 1990. Stemming from his early work in 1969 on transcutaneous uitrasonographic detection of air bubbles evaked by decompression (Spencer et al. 1969), he described a new application of TCD for detection of middle cerebral artery emboli during carotid endarterectomy (Spencer et al. 1990). Subsequent invesitigation of this interesting new technique validated the ability of TCD to detect microembolic signals, commonly known as MES, corresponding to both gaseous and solid microembolic materials (Mess and Hennerici 2001). Basically, the signal from an embolus passing through a sample volume ( $\mathrm{SV}$ ) of a pulsed Doppler system will add to the Doppler signal originating from the surrounding blood. This intensity increase causes a characteristic. sound, which can be described as a sudden "chirp"." snap" or exceptionally "moan"." The acousitic. information from an MES is so characteristic to the human ear, that after a short training period of several hours, a high level of interobserver agreement can be obtained.

Yet, the criteria of MES are poorly defined. Three basic properties of MES were mentioned by a consensus committee in 1995 (Consensus Committee of the Ninth International Cerebral Hemodynamics Symposium 1995). The MES are of short duration ( $<300 \mathrm{~ms}$ ), they exceed the background signal by at least $3 \mathrm{~dB}$, and, in the Doppler velocity spectrum analysis, they appear as unidirectional signals, at least if the MES is within the dynamic range of the Doppler system used. Especially the different ways to determine the intensity of the Doppler background signal in relation to its system depending nature (Markus and Molloy 1997) and the spontaneous variations of the Doppler background level (Wu and Shung 1996) render it rather impossible to define unambiguously the least intensity increase of an MES relative to the amplitude of the blood flow signal.

There are warious properties of the embolus itself which influence the appearance of the MES. Early animal or bench model studies (Markus et al. 1994; Markus and Brown 1993; Russell et al. 1991) show that MES caused by different particulate material, such as platelets or thrombi, have different amplitudes. However, since the backscattered power is also proportional to the amount of the material injected, it is not possible to reliably distinguish MES composed of different materials. The differentiation between particulate MES and those presumably consisting of air can be performed more reliably due to the relatively high amplitudes of the gaseous MES as compared to particulate MES (Georgiadis et al. 1994; Grosset et al. 1993; Markus et al. 1994; Markus and Brown 1993; Russell et al. 1991). This difference is attributable to the fact that solid material and air have quite different acoustic impedances. The reflecting properties of air MES were illustrated in a bench 
model experiment (Bumegin et al. 1994). Gaseous MES smaller than 0.5 . . detectable, whille those larger than $\mathbf{4 0}$ um were not measurable, due to signal saturation. Gaseous MES between 0.5 and $40 \mu \mathrm{m}$, however, display a linear relationship between size and spectral power.

It is generally accepted that MES originating from carotid artery plaques show the least intensity increase, whereas MES caused by gaseous bubbles produce the largest ones. The intensity increase of MES after CEA or due to atrial fibrillation is commonly slightly higher compared to those of carotid artery plaque MES. These differences imply that the latter type of MES is the most difficult to detect.

MES detection has been applied under numerous clinical circumstances, including carotid and intracranial artery disease, for cardiac sources of emboli like artificial heart valves, and monitoring procedures, e.g. during and after CEA. The number of MES can vary considerably depending on the source. lin a patient with a carotid artery plaque a frequency of 1 MES/hour can be encountered, whereas in a patient with an artificial heart valve even several hundred MES/hour might be measured. Nevertheless, it is commonly agreed, that healthy human beings do not have any MES at all. So, even the detection of one MES during a monitoring procedure lasting one hour will be pathologic.

Giaseous MES are regarded as relatively benign. Even thousands of gaseous MES measured during open heart surgery will only lead to neuropsychological deficits, that might resolve within months: after surgery (Pugsley et al. 1994). Particulate MES, on the contrary, have been related to neuronal damage, e.g. in animall models (Rapp et al. 2000). However, it is not clear, what the required size or quantity of particulate emboli is which is needed to cause cerebral infarction. Heistad et al. (1980) were able to show that alleady microspheres with a diameter of $50 \mu \mathrm{m}$ were sufficient to cause neuronal loss in an animal brain. Despite these uncertainties, some applications of MES detection have added to the understanding of pathophysiological processes, as is the case in patients with carotid artery disease. Though not unequivacally, it has been shown that certain plaque features as seen with ultrasound are associated with a relatively high number of MES, indicating an active process going on in the atherosclerotic lesion (Mess and Hennerici 2001). Yet, until now no clinical consequences have emerged from these data.

On the contrary, MES detection during and especially after CEA has conquered a place in routine patient management (Naylor 2002). If in a patient an excessive number of MES are detected postoperatively, the likelihood that neurological complications will occur, is increased manifold as compared to patients in whom no or only a modest amount of MES is found. Though it is not exactly known from what therapeutical interventions the patients will benefit most, the acministration of e.g. dextrane was shown to lower postoperative neurological complications considerably. MES typically evolve in the first two hours after CEA. This implies, that long-lasting monitoring sessions are required, which is expensive and strenuous for the observer counting the MES. So, an increasing need for reliable automatic MES detection system emerged and numerous approaches to this problem have been proposed. 
MES detection during CEA also provides relevant information, especially during the dissection phase, i.e. when the surgeon is approaching the carotid artery. Jansen et al. (1994) showed that a critical number of MES in the early phase of CEA was correlated with new postoperative cerebral lesions in the brain, as seen with magnetic resonance imaging (MRI). It appears that MES detection during dissection can guide the surgeon to awoid the release of emboli from the carotid artery plaque and hence make CEA safer (Naylor et al. 2000).

Moreover, TCD is well suited for the monitoring of cerebral blood flow during CEA (Naylor 2001). Doppleir systems observe the velocity of the blood flow at a high repetition frequency, allowing for a high temporal resolution (a few milliseconds). Since the diameter of the MCA is assumed to be fairly constant during the monitoring procedure, relative changes of the amount of blood flowing through the artery follow directly from the observed changes in blood flow velocity. A critical decrease of the values measured can directly be used to adapt the surgical strategy (Jansen et al, 1994, Ackerstaff et al. 2000). 50, TCD monitoring during and after CEA has proven to be clinically useful. Two studies including nearly 2.000 patients (Ackerstaff et al. 2000, Naylor et al. 2000) have shown that not only the hemodynamilc but also the embolic aspect can be adequately observed and corrected for if abnormal results are registered. 
Main objectives Automatic embolus detection systems, based on the analysis of the Doppler signal from a single sample volume, are already available, but their performance is not yet compared to human observers and in between. If a single gate system exhibits a low sensitivity and specificity then a more dedicated monitoring procedure should be considered. A possible drawback of a single gate system are intervening signal artefacts caused by motion, coughing etc.. A possible solution might be the analysis of signals from two sample wolumes. An artefact appears simultaneously in both sample volumes while emboli exhibit a time dellay, provided that both sample volumes cover the same artery.

A computer model relating sample volume position to local anatomy might indicate to what extent emboli are missed, even if emboli indeed pass through both sample volumes. They might be missed because the associated temporal rise in Doppler amplitude is obscured by spontaneous variations in blood cell arrangement and density. Moreover, the uneven sensitivity distribution is likely to cause a different appearance of the same embolus in both sample volumes. An optimized sample volume setting in conjunction with signal analysis techniques improving the temporal and spectral resolution (e.g. Wigner distribution) should improve sensitivity. The large size of the sample volume of TCD systems (length $10-20 \mathrm{~mm}$ and diameter $5-10 \mathrm{~mm}$ ) may also contribute to false detection of MES. Therefore, a conventional TCD was adapted to achieve the highest possible spatial resolution and to eliminate possible signal saturation. If a good spatial resolution can be obtained, then it should be possible to follow emboli through the circulation and relate changes in depth and velocity direction with the local anatomy.

Summarizing, the main objective of the thesis is to evaluate the interplay of MES appearence and their detectability with special emphasis on the moving nature of MES. 


\section{References}

Aaslid $R_{x}$ Markwalder $T M$, Nornes $H$. Noninwasive transcranial Doppler ultrasound recording of flow velocity in basal arteries. J Nieurosurg 1982; 57:769-74.

Ackerstaff RGA, Moons KGM, van de Vlasakker CWW, Moll FL, Vermeulen FEE, Algra A, Spencer MP. Association of intraoperative transcranial Doppler monitoring variables with stroke from carotid endarterectomy. Stroke 2000;31:1817-1823.

Barnett HJM, Taylor DW, Eliasziw M, Fox Al, Ferguson GG, Haynes RB, Rankin RN, Clagett BP. Hachinski VC, Sackett DL, Thorpe KE, Meldrum HE. Benefit of carotic endarterectomy in patients with symptomatic moderate or severe stenosis. N Engl J Med 1998;339:1415-142.5.

Bergman HL, Chesler NC, Ku DN, Wootton DM. Hemodynamics and atherosclerosis, in Hennerici M, Meairs S (eds): Cerebrovascular ultrasound; theory, practice and future developments. Cambridge, Cambridge University Press 2001 pp. 134-151.

Bunegin L, Wahl D, Albin MS. Detection and volume estimation of embolic air in the mididle cerebral artery using transcranial Doppler sonography. Stroke 1994;25:593-600.

Buys Ballot CHD. Bedrog van het gehoororgaan in het bepalen van de hoogte van een walargenomen toon. Caecilia.Algemeen Muzikaal Tijdschrift van Nederland 1845; Tweede jaargang, No.7:78-81.

Caplan LR. Brain embolism, revisited. Neurology 1993;43:1281-1287.

Caplan LR. Stroke" a clinical approach. Boston, Butterworth-Heinemann, 1993.

Caplan LR, Hennerici M. Impaired clearence of emboli (washout) is an important link between hypoperfusion, embolism, and ischemic stroke. Arch Neurol 1998;55:1475-148.2.

Derdeyn CP, Grubb RL, Powers WI. Cerebral hemodymamic impairment; methods of measurement and association with stroke risk. Neurology 1999:53:251-259.

Doppler C. Öber das farbige Licht der Doppelsterne und einiger anderer Gestirne des Himmels. Abh. Kgl. Böhm Ges Wissensch (Prag), 1842;465-482.

Evans $\mathrm{DH}_{3}$ MCDicken W/N. Doppler ultrasound; physics, instrumentation and signal processing, 2nd edition. Chicester: Wiley \&ons, 2000. 
Flanigan DP, Tullis JP, Streeter VL, Whitehouse WM, Fry WJ, Stanley JC. Multiple subcritical arterial stenoses: effect on poststenotic pressure and flow. Ann Surg 1977:186:663-668.

Freund H.J. Ultraschallregistrierung der Pulsation einzelner intrakranieller. Arterien zur Diagnostik von Gefäßverschlüssen. Arch Psychiatr Nervenkr 1965:207:247-253.

Gray.H. Anatomy of the human body.Philladelphia: Lea \& Febiger, 1918.

Grosset DG, Georgiadis D, Kelman AW, Lees KR. Quantification of ultrasound emboli signals in patients with cardiac and carotid disease. Stroke 1993:24:1922-1924.

Georgiadis D, Mackay TG, Kelman AW, Grosset DG, Wheatley DJ, Lees KR. Differentiation between gaseous and formed embolic materials in vivo. Stroke 1994:25:1559-1563.

Gnasso A, Carallo C, Irace C, Spagnuolo V, De Novara G, Mattiolo PL, Pujia A. Association between intima-media thickness and wall shear stress in common carotid artery in healthy male subjects. Circ 1996;94:3257-3262.

Heistad DD, Marcus ML, Busija DW. Measurement of cerebral blood flow in experimental animals with microspheres: applications of the method, in Passonneau JN, Hawkins RA, Lust WD, Welsh FA (eds): Cerebral metabolism and neural function. Baltimore, Williams and Wilkins, 1980, pp. 202-211.

Heninerici MG. High intensity transcraniall signals (HITS): a questionable 'jackpot' for the prediction of stroke risk. I Heart Valve Dis. 1994;3:1 24-5.

Jansen C. Ramos LMP, van Heeswijk JPM, Moll FL, van Gijn ل, Ackerstaff RGA. Impact of microembolism and hemodynamic changes in the brain during carotid endarterectomy.

Stroke $1994 ; 25: 992-997$.

Lang J, Busche KA al, Buschimann W, Linnert D. Kopf, Teil B, in won Lanz T, Wachsmuth W (eds): Praktische Anatomie. Berlin, Springer, 1979.

Markus HS, Brown MM. Differentiation between different pathological cerebral embolic matterials using transeranial Doppler in an in vitro model. Stroke 1993;24:1-5.

Markus H, Loh A, Brown MM. Detection of circulation cerebral emboli using Doppler ultrasound in a sheep model. J Neurol Sci 1994:122:117-124. 
Markus HS Molloy J. Use of a decibel threshold in detecting Doppler embolic signals.

Stroke $1997,28: 692-695$.

Mess WH, Titulaer BM, Ackerstaff RGA. An in vivo model to detect microemboli with multidepth technique. Preliminary results (abstract). Cerebrovasc Dis 1996;6;suppl.3:60.

Mess WH, Henneric MG. High Intensity Transient Sigmals, in Hennerici M, Meairs S (eds): Cerebrovascular Ultrasound; Theory, Practice and Future Developments. Cambridge, Cambridge University Press, 2001, pp, 297-316.

Naylor AR, Hayes PD, Alroggen H, Lennard N, Gaunt ME, Thompson MM, London NMM, Bell PRF. Reducing the risk of carotid surgery: a 7-year audit of the role of monitoring and quality control assessment. J Vasc Surg 2000;32:750-759.

Naylor AR. Transcranial Doppler monitoring during carotid endarterectomy, in Hennerici M, Meairs 5 (eds): Cerebrovascular Ultrasound; Theory, Practice and Future Developments. Cambridge, Cambridge University Press, 2001, pp.317-323.

Naylor AR. Regarding "high embolic rate early after carotid endarterectomy is associated with early cerebrovascular complications, especially in women "invited comment). J Vasc Surg 2002;36:408-409.

Pearce WH, Parker MA, Feinglass J, Ujiki M, Manheim LM. The importance of surgeon volume and training in outcomes for vascular surgical procedures. J Vasc Surg 1999;29:768-778.

Pugsley $W_{i}$ Klinger L, Paschalis C. Treasure T, Harrison $M_{f}$ Newman S. The impact of microemboli during cardiopulmonary bypass on neuropsychological functioning. Stroke 1994;25:1393-1399.

Rapp JH, Pan XM, Sharp FR, Shah DM, Wille GA, Velez PM, Troyer A, Higashida RT, Saloner D. Atheroemboli to the brain: size threshold for causing acute neuronal cell death. J Vasc Surg 2000:32:68-76.

Ross R. Atherosclerosis - an inflammatory disease. N Engl I Med 1999;340:115-126.

Rothwell $P M$, Warlow $C$. Prediction of benefit from carotid endarterectomy in individual patients: a risk-modelling study. Lancet 1999;353:2105-2110.

Russell D, Madden K., Clark WM, Sandset PM Zivin JA. Detection of arterial emboli using Doppler ultrasound in rabbits. Stroke 1991;22:253-258. 
Spencer MP, Cambell SD, Sealy $L_{i}$ Henry FC, Lindbergh J. Experiments on decompression bubbles in the circulaltion using ultrasonic and electromagetic flow meters, 1 occup Med 1969/1/238-44.

Spencer M P, Thomas GI, Nicholls SC, Sauvage LR. Detection of middle cerebral artery emboli during carotid endarterectomy using transcranial Doppler ultrasonography. Stroke 1990,21*415-23.

Vainas T, Kurvers HAMM, Mess WH, de Graaf R, Ezzahiri R, Tordoir JHM, Schurink GWH, Bruggeman CA, Kitslaar PJEHM. Chlamydia pneumoniae (Cpn) serology is associated with thrombosis-related but: not with plaque-related micro-embolization during carotid endarterectomy. Stroke 2002;33: 1249-1254.

Warlow CP, Dennis MS, van Gijn J, Hankey GJ, Sandercock PAG, Bamford JM, Wardlaw JM. Stroke; a practical guide to management, 2nd edition. Oxford: Blackwell Science, 2001.

Wu SJ, Shung KK. Cyclic variation of Doppler power from whole blood under pulsatile flow. Ultrasound Med Biol 1996:22:883-894. 


\section{List of abbreviations}

ACA anterior cerebral artery

Acom $A$ anterior communicating artery

CCA common carotid artery

CEA carotid endarterectomy

ECA external carotid artery

EEG electroencephalogram

ICA internal carotid artery

LDL. low density lipoprotein

MCA middle cerebral artery

PCA posterior cerebral artery

PcomA posterior coinmunicating artery

SSEP somato sensory evoked potentials

TCD transcranial Doppler sonography

TIA transient ischemic attack 
2

Automatic embolus detection compared with human experts; a Doppler ultrasound study Stroke 1996;27:1840-1843 

Abstract Transcranial Doppler ultrasound (TCD) reliably detects the occurrence of microembolic signals (MES). Unfortunately, TCD monitoring is a time-consuming and mentally strenuous procedure. The purpose of this study was to assess whether automatic embolus detection solfware devices acting as a "stand-alone systiem" are able to identify MES in patients with solid cerebral microemboli. Ten records of TCD monitoring of the middle cerebral artery in patients with symptomatic highgrade carotid artery stenosis were analyzed for the moments at which MES occurred by four observers and three automatic detection software devices (RB11 on TC2000. Pioneer Version 2.10. and Embotec). The resuits of the three software systems were assessed on the basic assumption that MES were present if at lleast three of the four observers agreed. The average number of 1-5econd periods in which MES were detected by the four observers per tape ranged from 5 to 39 . The overall $\kappa$ values (and SES) for chance-corrected interobserver agreement between the four observers ranged from $.94(.02)$ to $99(.01)$.

The agreement between the software devices and the observers was lower, with $\mathrm{K}$ values (and SEs) ranging from .18 (.17) to $.93(.07)$. The RB11 and Embotec systems achieved a $k$ value higher than 0.4 in all tapes. The Pioneer system failed to reach a $k$ value of 0.4 in three tapes. The RB11 showed a sensitivity of $70 \%$ for detecting MES, the Embotec $62 \%$, and the Pioneer $44 \%$.

In patients with symptomatic high-grade carotid artery stenosis, a high degree of agreement in the detection of moments of MES can be achieved between observers. The three automatic detection software devices reached less agreement. Supervision of TCD monitoring and assessment of MES by an experienced observer is still necessary. 
Introduclion The search for parthophysiological factors of cerebral ischemia has recently led to the introduction of microembolus detection by transcranial Dopplersonography (TCD) (Spencer 19921. This technique reliably detects the occurrence of microembolic signals (MES) that clearly differ from the normal bload flow spectral waveform.

The similarity between these Doppler signals in patients and in animals in which embolic material had been introduced suggests that MES represent embolic particles (Russell et al. 1991, Markus et al. 1994, Kessler et 1.1992). Furthermore, there is a strong correlation between the number of MES in the middle cerebral artery (MCA) and symptomatic atherosclerosis of the ipsilateral carotid artery (Siebler et al. 1992, Grosset et al. 1994, Siebler et al. 1994a, Ries et al. 1995, Van Zuilen et al. 1995). Unfortunately, TCD monitoring is a time-consuming and mentally strenuous procedure. The large number of patients who are candidates for this examination has led to the development of systems ailmed at detecting MES automatically with rejection of artifacts or other changes of the Doppler signal. The purpose of this study was to assess whether automatic embolus detection software systems acting as "stand-alone systems" can adequately identify MES in patients with cerebral microemboli associated with carotid artery stenosis. The results of these automated analyses were compared with the separate assessment of MES by human experts, which is still regarded as the most accurate method of microembolus detection. 
Materials and Methods Ten different records of TCD monitoring of the MCA in as many patients with an ipsilateral symptomatic high grade carotid artery stenosis were analyzed. TCD monitoring was performed by means of a TC2-64 (EME) with a 2-MHz monitoring transducer. The monitoring periods were recorded on the audio channels of a stereo VHS recorder and lasted 30 minutes, with the exception of tapes 3 and 9 with 20 minutes of recording. The tapes contained different numbers of MES as well as varlous types of artifacts such as probe movements, speaking; and snoring.

Four observers (E.V.Van Z., W.H.M., C.J., R.G.A.A.) independently performed analysis of the tapes for the occurrence of MES using a TC2000 (EME). This TCD system was equipped with a 386 processor and used a 128-point color-coded fast Fourier transform (FFT). Scale settings were as close as possible to the settings of the original Doppler recordingls. The sweep was kept constant and corresponded with a time window overlap of $46 \%$ or $66 \%$, depending on the velocity scale. Specially designed software (EME) was used for determining duration, frequency, and power characteristics of the Doppler signals. According to established criteria (Consensus Committee of the Ninth International Cerebral Hemodynamics Symposium 1995), MES were identified on the basis of their typical musical sound, a duration of less than 300 milliseconds, an amplitude exceeding the background signal by at least $3 \mathrm{~dB}$, and a unidirectional appearance within the Doppler velocity spectrum. The actual moments at which MES occurred were identified, instead of the absolute number of signais on each tape.

The 10 tapes were also analyzed by three automatic detection software systems: RB 11 software on the TC 2000 (EME), automatic event detection softwale (version 2.10) on the Pioneer (EME), and the neural network of Embotec (STAC). All software systems were used with the standard settings of the latest available versions, especially with pegard to embolus detection. The gain of the input signal was adjusted at the beginning of the anallysis and, if necessary, readjusted during the analysis to achieve the best possible envelope of the Doppler spectrum. Since all MES were within the dynamic range of the TC2-64 Doppler system, overload of the preamplifier could be excluded as a cause of technical problems of automated embolus detection (Markus et all. 1993). The software analysis was performed only once, because this resembles clinical practice.

\section{Description of the Automatic Detection Software Systems}

\section{RBD1 [EME]}

The RB11 software on the TC2000 provides a computerized embolus detection algorithm, which calibrates itself at the start of the examination. The trigiger level and artifact rejection level are set according to the power of the Doppler signal. The algorithm relies on the characteristic bell-shaped increase in the relative power occurring with an embolus. If the power ratio between a highintensity signal and the background signall exceeds the trigger level and if the difference between the maximum power above and below the zero line remains below the artifact rejection level, the signal will be counted as an embolus. If the second condition is not fulfilled, the signal is classified as an artifact. 
Pioneer Version 2.10 IEME

This device includes software that counts "events, "defined as a sudden increase in the power of the ultrasound signal expressied in decibels, by means of two algorithms. If a signal exceeds the minimal threshold level, it is further calculated by the artifact rejection algorithm. This algorithm compares the signal intensity above and below the zero line with the mean background signal. Signals that exceed the artifact rejection level are omitted; the other signals are counted as events.

\section{Embotec ISTAC)}

A. three-layer neural network, consisting of 512 input neurons, 10 hidden-layer neurons, and 3 output neurans is trained through a database of FFT Doppler spectra. These Doppler spectra are analyzed for the presence of MES by human observers. The 3 output neurons correspond to the network decisions "microembolus," "artifact," or "normal." The output value of "microembolus" represents a network estimation of the similarity in FFT between an input signall and the MES in the database.

\section{Statistical Analysis}

To localize the moments at which MES occurred, the running time of the tapes was subdivided into periods of 1 second. The agreement of MES between the four observers was assessed with Cohen's $\kappa$ values, Cohen's $\kappa$ provides a correction for chance agreement, which makes it superior to other indices of interobserver agreement. $\kappa$ values may vary between 1 (complete agreement) and - 1 (complete disagreement); zero represents agreement similar to that expected by chance.

A common guideline is that a $\kappa$ value of .75 or more indicates excellent agreement, whereas $\kappa$ values from 4 to .75 indicate fair to good agreement and values of .4 or less poor agreement (Fleiss 1981). $\kappa$ values only slightly depend on the number of correct negative counts, which is mostly high in these studies.

Agreement between the observers was considered the "gjold standard" for the automated procedures. Comparison with the three software systems was performed on the basic assumption that a moment of MES was present if at least three of the four observers agreed. All other moments of MES detected by the software systems were considered fallse-positive. For each system the sensitivity (true-positive rate) was calculated for each of the 10 tapes and overall. Because of the high proportion of correct inegative MES in this study, the specificity would be unrealistically high and the false-positive rate very low. Instead, the false-positive moments of MES were expressed as the proportion of the sum of true-positive and false-positive moments of MES detected by the software systems. Furthermore, $k$ values were calculated to assess the agreement between any of the three software systems and the human gold standard. For each of the human and artificial observers, a $\chi^{2}$ test was used to evaluate the hypothesis that the $\kappa$ values in the separate tapes are equal (Fleiss 1981). 
ReSUlts The four observers found a mean number of moments of MES (and SD) ranging from $5(8)$ on tape 6 to $39(.5)$ on tape 4. The $k$ values and their SEs for the agreement between the observers in each of the 10 tapes are given in table 1 . The overall $\kappa$ values and $5 E$ s ranged from $.94(.02)$ to $.99(.01)$. The $\chi^{2}$ test showed no differences in $\kappa$ values between the separate tapes in each of the four obserwers. Table 2 lists the results of the comparison between the human gold standard (agreement on moments of MES between at least three observers) and the three automatic embolus detection software systems. The agreement between the software devices and the obserwers: collective was lower than the agreement between the observers $s_{r}$ with $\kappa$ values (and SEs) ranging from $.18(.17)$ to $.93(.07)$. The RB11 and Embotec achieved a $k$ value above. 4 in all 10 tapes, whereas the Pioneer failed to reach a $\kappa$ value of 4 in three tapes. For each of the three software systems, the $\chi^{2}$ test showed a significant difference in $\kappa$ walues between the separate tapes (RBI1 $P<.001$, Pioneer $P<.001$, Embotec $P<.05$ ).

The RB1 1 achieved an overall sensitivity of $70 \%$ for detecting moments with MES for all tapes, the Embotec $62 \%$ and the Pioneer $44 \%$. The figure illustrates the number of false-positive moments of MES calculated as a percentage of the sum of true-positive and false-positive moments of MES detected by the three software systems in each tape. The RB 11 detected more false-positive moments of MES than true-positive moments of MES in tapes 2 and 8 , as did the Pioneer and Embotec in tape 5 .

\section{Table 1}

א. Values of chance-corrected agreement on the moments at which MES occurred between the four observers in each af the 10 tapes and in overall analysis

\begin{tabular}{|c|c|c|c|c|c|c|}
\hline \multirow{3}{*}{$\begin{array}{l}\text { Tape } \\
1\end{array}$} & \multicolumn{6}{|l|}{ Observers } \\
\hline & $1 \rightarrow \rightarrow 2$ & $1 \leftrightarrow 3$ & $1 \leftrightarrow 4$ & $2 x \rightarrow 3$ & $2 \rightarrow 4$ & $3 \leftrightarrow \rightarrow 4$ \\
\hline & $.86(.06)$ & $95 \quad(.04)$ & $92 \quad(.05)$ & $.82(.07)$ & $.78 \quad(.08)$ & $.87(.06)$ \\
\hline 2 & 1 & $.97 \quad(.03)$ & $.97 \quad(.03)$ & $.97(.03)$ & $.97 \quad(.03)$ & $.94(.04)$ \\
\hline 3 & $.87 \quad(.08)$ & 1 & $.80 \quad(.1)$ & . 67 (.08) & $.84 \quad(00)$ & $.8 \quad(.1)$ \\
\hline 4 & $.99 \quad(.011)$ & $97 \quad(02)$ & $.95 \quad(.08)$ & $.96 \quad 6021$ & $94 \quad(.03)$ & $.958 .03 \%$ \\
\hline 5 & H & $96(.04)$ & $.96 \quad(.04)$ & $.96(.04)$ & $.96(.04)$ & $.92 \quad 0.06$ \\
\hline 6 & $.91 \quad(.09)$ & $.89(.111$ & 1 & $.8 \quad(14)$ & $.91 \quad(.09)$ & .894 .111 \\
\hline 7 & $.92 \quad(.08)$ & $.91(09)$ & $\Uparrow$ & $.83(12)$ & $.92 \quad .08)$ & $91 \quad 1,091$ \\
\hline 8 & $.95 \quad(.05)$ & $\|$ & $.95 \quad(0,5)$ & $.95 \quad(.05)$ & $.90(.07)$ & $.95 \quad(05)$ \\
\hline 9 & $.94 \quad(.06)$ & $\|$ & 1 & $.94 \quad(.06)$ & $.94 \quad(06)$ & 1 \\
\hline 10 & $.97 \quad(.03)$ & 1 & .97 (..03) & $.97(03)$ & $.94 \quad(.04)$ & $.97(.03)$ \\
\hline A.ll & $.99(.01)$ & $.99(.011)$ & .98 (an) & $.26(01)$ & $.94 \quad(.02)$ & $.96(01)$ \\
\hline
\end{tabular}

Walues in parentheses are SEs. 
Discussion The present study shows that for the detection of moments with MES in symptomatic high-grade carotid artery stenosis, a high degree of interobserver agreement can be achieved between experienced observers. This confirms the reproducibility of a technique that might become standard in the evaluation of cerebrowascular disease. Recently, good agreement has been reported between human and computerized analysis of MES (Markus et al. 1993, Siebler et al. 1994b, Georgiadis et al. 1995). In contrast, we found less agreement between the automatic embolus detection software devices and the human experts. One reason for this disparity might be that in our study only patients with high-grade carotid artery stenosis were included. Most of the earlier studies have been done in patients with prosthetic heart valves and under in vitro conditions, with relatively large experimental emboli; in these circumstances MES have a higher intensity and a longer duration than in patients with carotid artery disease (Georgiadis et al. 1994). The temporal resolution of the automatic detection devices might not be high enough to detect all shortduration low-intensity MES derived from microemboli in carotid artery disease (Markus 1995), whereas human observers have the advantage of hearing the typical sound characteristics of a microembolus in the audio Doppler signal. In the present study the percentage of time window overlap was not always sufficient to visualize all MES on the spectral display. Therefore, it might be expected that human observers reach better agreement.

Another reason that our results differ from those in previous studies (Siebler et al. 1994b, Georgiadis et al. 1995) on interobserver agreement might be that we identified the actual moments of MES on the tapes rather than the absolute number of MES. This method was chosen to ensure that the measurement of agreement is based on identification of the same signals, and we argue that this approach is the most correct in assessing the performance of an automatic embolus detection device. For example, the RB 11 indicated a total number of 159 MES that consisted of 101 truepositive counts and 58 false-positive counts; according to the observers' collective, the actual number of MES was 1.45 (Table 2). Otherwise, one might have concluded that all the actual MES as well as an additional if if ise-positive ones were detected. Obviousily, this would resuit in markedily different conclusions about the accuracy of this embolus detection device.

The automatic detection software systems failed to achieve high sensitivity. However, it is not really important to produce artificial systems that detect MES with $100 \%$ sensitivity as long as the detection rate of MES is reproducible. The RB11 detected more than half of the moments with MES in all tapes, and the Embotec failed in only one tape, thus providing useful information about the presence or absence of these signals as well as a reasonable estimate of the absolute number in most of the tapes. In contrast, the Pioneer in its default settings missed more than $50 \%$ of the moments of MES in half of the tapes.

The three software devices use different methods for embolus detection. The Embotec neural network is capable of classifying patterns after learning typical examples from a database (Siebler et al. 1994b). Therefore, the system is biased and especially set at identifying MES that are identical to those from the training database. The RB11 has been developed in in vitro conditions (Brucher and Russell 1993). MES are predominately recognized by their typical bell-shaped increase in relative 
Table 2

Number of moments af MES and false-positive moments of microenbolic signals, sensitivity, $k$ watue, and $5 E$ of interobsent

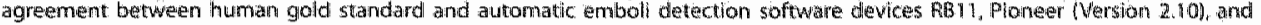
Embolec

\begin{tabular}{|c|c|c|c|c|c|c|c|c|c|c|c|c|c|c|c|c|}
\hline \multirow[b]{2}{*}{ Tape } & \multirow[b]{2}{*}{ HGSn } & \multicolumn{5}{|c|}{$\mathrm{RB} 11$} & \multicolumn{5}{|c|}{ Pioneer } & \multicolumn{5}{|c|}{ Embotec } \\
\hline & & $n$ & EPIn] & Senspol & $\kappa$ & $S E$ & in & FPln: & Sens & $\pi$ & $S E_{0}$ & $n$ & FPIn] & Sens[\% & $x$ & $5 \mathrm{E}$ \\
\hline 1 & 19 & 71 & 2 & 58 & .68 & 09 & 5 & 1 & 26 & 40 & .13 & 11 & $i$ & 58 & 71 & .09 \\
\hline 2 & 18 & 13 & 14 & 72 & .57 & .09 & 11 & 1 & 61 & .73 & .09 & 13 & 3 & 72 & .76 & .09 \\
\hline 3 & 10 & 8 & 0 & 80 & .89 & .09 & 4 & 0 & 40 & .57 & .16 & 6 & 1 & 60 & .70 & .13 \\
\hline 4 & 38 & 26 & 20 & 68 & .61 & .06 & 113 & 4 & 34 & .47 & .08 & 19 & 4 & 50 & 62 & 07 \\
\hline 5 & 12 & 8 & 0 & 67 & 80 & .09 & 7 & 45 & 58 & .21 & .07 & 5 & 6 & 42 & .43 & .93 \\
\hline 6 & 5 & 3 & 0 & 60 & .75 & .17 & 1 & 0 & 20 & .33 & .25 & 3 & 0 & 60 & .75 & .17 \\
\hline 7 & 6 & 6 & 5 & 100 & .75 & .12 & 4 & 0 & 67 & .80 & .114 & 5 & 1 & 83 & .83 & .12 \\
\hline 8 & 10 & 8 & 15 & 80 & 48 & .11 & 1 & 0 & 10 & .18 & .17 & 7 & 7 & 70 & .58 & .12 \\
\hline 9 & 8 & 5 & 1 & 63 & .71 & .14 & 5 & 0 & 63 & .77 & .13 & 7 & 0 & 86 & .93 & .07 \\
\hline 10 & 19 & 13 & 1 & 68 & .79 & .08 & 13 & 0 & 68 & .81 & .07 & 14 & 2 & 74 & .80 & $.0 \%$ \\
\hline All: & 145 & 101 & 58 & 70 & $\ldots$ & $\ldots$ & 54 & 51 & 44 & $\ldots$ & $\ldots$ & 90 & 25 & 62 & $\ldots$ & $\ldots n+1$ \\
\hline
\end{tabular}

HGS indicates humang gold standard (see text); FP, fal se-positive moments of MES, and Sens, sensitivity.

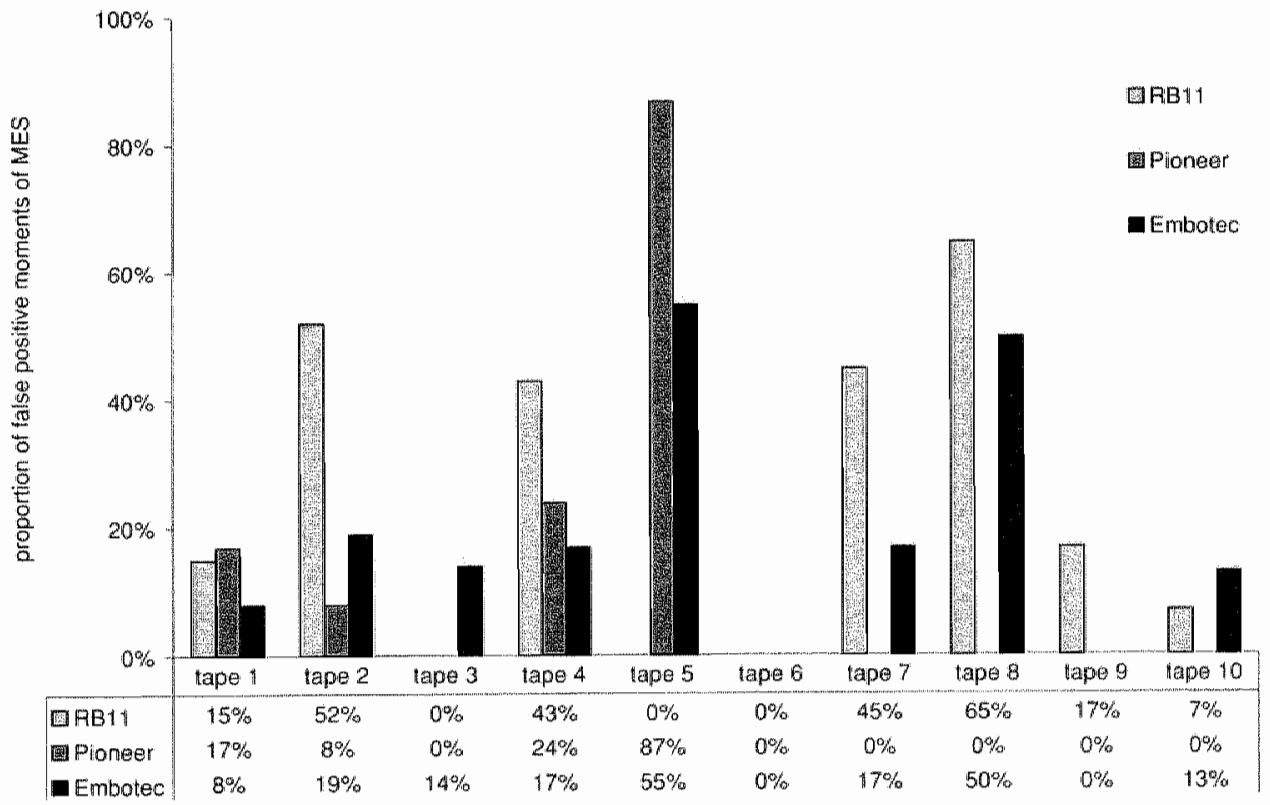

Figure $\|$

The number of false positive moments of MES represented as a percentage of the rotal number of true and false positive moments of MES detected by the three sof tware systems in each of the 10 tapes. 
power. The larger proportion of false-positive MES dietected by this device compared with the other two systerns suggests difficulties in distinguishing the spectral characteristics of emboli from those of artifacts. The Ploneer uses a less sophisticated detection algorithm but showed a favorably low proportion of false-positive MES. All software systems provide the possibility of changing the settings of the detection algorithm parameters: we used only the standard settings.

Although in this studly the three software devices for automatic embolus detection reached less agreement than the four human experts, the results are promising, and it is likely that ongoing research and development will further improve the performance of the systems. At minimum, they may be important to help reduce the vast amount of data. In future designs of TCD equipment and embollus detection software, a high temporal resolution is an important feature. The Wigner signal analysis might be a useful alternative ( 5 mith et al. 1995) The present study shows that supervision of TCD monitoring and assessment of MES by experienced observers is still necessary. 


\section{References}

Brucher R, Russell D. Automatic embolus detection with artifact suppression. J Neuroimaging 1993:3:77. Abstract.

Consensus Committee of the Ninth International Cerebral Hemodynamic Symposium.

Basic identification criteria of Doppler microembolic signals. Stroke 1995:26:1123.

Fleiss JL. Statistical methods for rates and proportions. In: Statistical Methods and Proportions. 2nd ed. New York, NY: J Wiley \& Sons; 1981:212-225.

Georgiadis D, Mackay TG, Kelman AW, Grosset DG, Wheatley DI, Lees KR. Differentiation between gaseous and formed embolic materials in vivo Stroke 1994:25:1559-1563.

Georgiadis D, Kaps M, Siebler M, Hill M, Konig M, Berg J, Kahl M, Zunker P, Diehl B, Ringelstein EB. Variability of Doppler microembolic signal counts in patients with prosthetic cardiac valves. Stroke 1995;26:439-443.

Grosset DG, Georgiadis. D, Abdullah I, Bone I, Lees KR. Doppler emboli silgnals vary according to stroke subtype. Stroke 1994;25:382 384.

Kessler C, Kelly AB, Suggs WD, Weissman JD, Epstein CM, Hanson SR, Harker LA. Induction of transient neurological dysfunction in baboons by platelet microemboli. Stroke 1992;23:697-702.

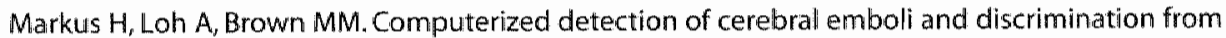
artifact using Doppler ultrasound. Stroke 1993;24:1667-1672.

Markus $\mathrm{H}_{2}$ Loth A, Brown MM. Detection of circulating cerebral emboli using Doppler ultrasound in a sheep madel. J Neurol Sci 1994;122:117-124.

Markus $\mathrm{H}$. Importance of time-window overlap in the detection and analysis of embolic signals. Stroke 1995;26:2044-2047.

Ries S, Schminke U, Daffertshofer M, Schïndlmayr C. Hennerici M. High intensity transient signals and carotid artery disease. Cerebrovasc Dis 1995;5:124-127.

Russell D, Madden KP, Clark WM, Sandset PM, Zivin JA. Detection of arterial emboli using Doppler ultrasound in rabbits. Stroke $1991,22: 253-258$. 
Siebler M, Sitzer M; steinmetz H. Detection of intracranial emboli in patients with symptomatic extracranial carotid artery disease Stroke 1992;23:1652-1654.

Shebler $M$, Kleinschmidt $A$, Sitzer $M_{t}$ Steinmetz $H$, Freund $H J$. Cerebral microembolism in symptomatic and asymptomatic high-grade internall carotid artery stenosis. Neurology $1994 a, 44615-618$.

Siebler M, Rose G, Sitzer M, Bender A, Steinmetz H. Real-time identification of cerebral microemboli with US feature detection by a neural network. Radiology 1994b; 192:739-742.

Smith JL, Evans DH, Fan L, Gaunt ME, London MJN, Bell PRF, Naylor AR. Interpretation of embolic phenomena during carotid endarterectomy. Stroke 1995;26:2281-2284.

Spencer MP. Detection of cerebiral arterial emboli. In "Newell DW, Aaslid R, eds. Transcranial Doppler. New York, NY: Rawen Pres5; 1992:215-230.

Van Zuilen EV, Mauser HW, Algra A, Van Gijn J, Ackerstaff RGA.TCD detection of microemboli in symptomatic and asymptomatic high-grade carotid artery stenosis. (Abstract) J Neuroimaging 1995; suppl 2:\$63. 
3

Middle cerebral artery anatomy and characteristics of embolic signals: a dual gate computer simulation study

Ultrasound Med Biol 1999;25:531-539 

Abstract In terms of microembolic signal (MES) detection the anatomy of the middle cerebral artery (MCA) mainstem has only scarcely been considered. The vessel itself, howewer, could be at least partly responsible for the enormous variation when calculating the essential time difference ( $\triangle \mathrm{t}$ ) values of MES using the dual-gate technique. Therefore, we studied the time characteristics of MES in a computer simulation applying an anatomically realistic vessel and a dual-gate TCD approach. Three different MCA amatomies and two MES to blood intensities were simulated as well as two different sample volume settings. The MES length (proximal sample volume $t_{\text {; }}$ idistal sample volume $t_{2}$ ) and $\Delta t$ were calculated for different angles of insonation and sample wolume depths. The calculations of the time characteristics of MES showed extreme variation, with only modest changes of the insonation angle $\left(t_{1} 4-34 \mathrm{~ms}_{;} \Delta t 9-27 \mathrm{~ms}\right)$ or the sample volume depth $\left(\mathrm{t}_{1} 7-27 \mathrm{~ms}_{\text {; }}\right.$ $\Delta t$ 6-32 ms). The variation could be considerably reduced with modified TCD settings i.e., a shorter gate separation combined with a shorter receiver gate time in the distall sample volume ( $\Delta t$ with changing insonation angles 6-19 $\mathrm{ms}$; $\Delta \mathrm{t}$ with changing insonation depths $13-17 \mathrm{~ms}$ ). These results mot only urge us to a cautious interpretation of the properties of single MES, but also contribute to an understanding of the marked $\Delta t$ variation using the dual-gate technique. 
Introduction Transcranial Doppler (TCD) ultrasound reliably detects the occurrence of microembolic signals (MESs) in the middle cerebral artery (MCA). Recently, several investigators have been able to demonstrate the clinical usefulness of this method in relation to carotid endarterectom A correlation between the number of MESs during dissection of the carotid artery and postoperatively new neurological deficits confirmed by new MRIl lesions could be demonstrated (Ackerstaff et al. 1995). Gaunt et al. (1994) showed that MESs at this stage of the endarterectomy were associated with postoperative neuropsychological disorders. Levi et al. (1997) found a high correlation between the number of MESs postoperatively and the probability of a cerebrovascular event in the first month after surgery. Manual TCD monitoring for detection and counting of MESs is time consuming and difficult, and is ideally performed using an automated system. We tested three commercially available automatic emboli detection systems (van Zuilen et al. 1996). The results were promising, but not completely satisfactory. It was puzzling to see the automated systems missing MESs that were clearly identifiable by the human ear. A potential candidate for an improved automatic detection system is the dual-gate technique (Georgiadis et al. 1996; Mess et al. 1996): a moving particle in the MCA, in contrast with an artefact, should have a transit time between two sites of detection ("transit-time hypothesis"). This time difference ( $\Delta \mathrm{t})$ could then be measured automatically and used as a criterion for acceptance or rejection of an intensity increase as an MES.

We tested this transit-time hypothesis (Mess et al. 1996) in a human model and found a surprisingly large range for the $\Delta t$ values (-3 to $31 \mathrm{~ms}$ ). Smith et al. (1997a) also reported on "curious observations" regarding signalis from dual-gated TCD systems, including the great variability of $\Delta$ t. However, an automatic embolus detection system partly based on the transit-time hypothesis needs $\Delta t$ values in a predictable range. So, more has to be known about the factors that influence the appearence of an MES in the raw data.

The effects of different Doppler parameters (ultrasound pulse amplitude, gain and sample volume burst length) on MES appearence have been analyzed (Droste et al. 1994a), as well as the influence of the MES velocity on its time characteristics. (Droste et al. 1994b), but the anatomy of the MCA mainstem has only scarcely been considered. Sketches explaining the "transit-time hypothesis" often show a quite long MCA that easily allows two sample wolumes to be placed behind each other, even with some space in between (Georgiadis et al. 1996). Anatomical studies, on the contrary, showed that the MCA mainstem, which is the site of emboli detection, has a mean length of only $16 \mathrm{~mm}$ (range $5 \mathrm{~mm}$ to $31 \mathrm{~mm}$ ) with $8 \%$ of the measured MCAs even shorter than $10 \mathrm{~mm}$ (Jain 1964; Lang et al. 1979). Angiographic studies underlined, moreover, that the MCA is not a straight tube, but variably curved (Huber et al.1979). Both the transected length and the angle of insonation are, thus, very likely to be different for two sample volumes, when placed serially using typical settings.

The MCA anatomy is likely to contribute considerably to the enormous variation of the essential $\Delta t$ values. We, therefore, developed a computer model enabling us to simulate a dual-gated TCD system and different anatomical situations. in the recent work of Smith et al. (1997a), a theoretical 
(i.e, computer) model for dual-gated TCD was also presented. However, their focus was on the interaction between the two sample wolumes and MESs of different intensity traveling in a straight tube at different angles of insonation. The aim of our studly was to emphasize the effects of anatomically realistic vessels on the MES appearance when changing sample volume depths and insonation angles. Additionally, we compared two TCD settings with a different gate separation and a different receiver gate time of the distal sample wolume with all other parameters kept constant, to test the hypothesis that the wariation of the $\Delta t$ values can be reduced by adjusting the sample volume positions. This would be a crucial step toward a reliable automatic MES detection system based on the dual-gate technique. 
Methods and materials First, a computer simulation model will be described and second, the tests we performed with this model will be elucidated.

\section{The simulation madel}

We developed a three dimensional (3-D) computer model basied on a programmable signallanalyzing software (Testpointo) installed on a personal computer. Basically, the model mimicked an MCA and two sample volumes of a TCD. It allowed simulation of the appearance of MESs in the sample volumes while they were moving through the vessel. The details concerning the vessel, the TCD and the MES are as follows:

The vessel

The modeled vessel comprised three anatomical structures: the distal internal carotid artery, the mainstem of the MCA (M1) and the distal MCA (M2). This mock vessel could be adjusted in the coronal (anterior-posterior view) and axial (or horizontal) planes, allowing the modeling of a wide range of MCA anatomies in three orthoganal dimensions. Figure $1 \mathrm{a}$ and $\mathrm{b}$ displays examples of a vessel and the position of the two sample volumes.

The TCD

A dual-gate system was simulated with two sample volumes covering the MCA, as shown in Fig. 1. The lateral sensitivity of the sample volumes was calculated applying waterbath data of a $2-\mathrm{MHz}$ probe provided from EME, Kleinostheim, Germany (see Fig. 2a). This acoustic pressure plot was transformed to an isoamplitude mapping, comparable to those that were found by Arnolds et al. (1989) for a $2-\mathrm{MHz}$ probe of the same manufacturer. We assumed that the axial sensitivity for the depth used was constant in terms of probe characteristics and only depended on the sample volume burst length and the receiver gate length. An example of the resulting analytical fit is given in Fig. 2b. The data were normalized with the highest amplitude having a value of 1 .

Distortions of the isoamplitude mapping due to the human skull were not taken into account. Theoretically, these should have an effect, especially on the borders of the sample volume where the sensitivity is low. In practice, these variations cannot be assumed to be constant between different human beings, and will depend unpredictably on the exact structure of the insonated skull.

\section{The embolus}

The MES was assumed to follow the course of the modeled vessel with a constant velocity $140 \mathrm{~cm} / \mathrm{s}$ throughout the study). The flow was regarded as being laminar, with the MES always in the center of the vessel. We choose this approach to focus on the influence of the vessel anatomy an the time characteristics of MESs. Incorporating exact flow fields, turbulence, and vessels branching off (e.g., with computational fluid dynamics) would result in a lot more assumptions on wessel diameter, wall shear rate of the insonated vessel, viscosity of the blood and cardiac pressure. Especially in case of 


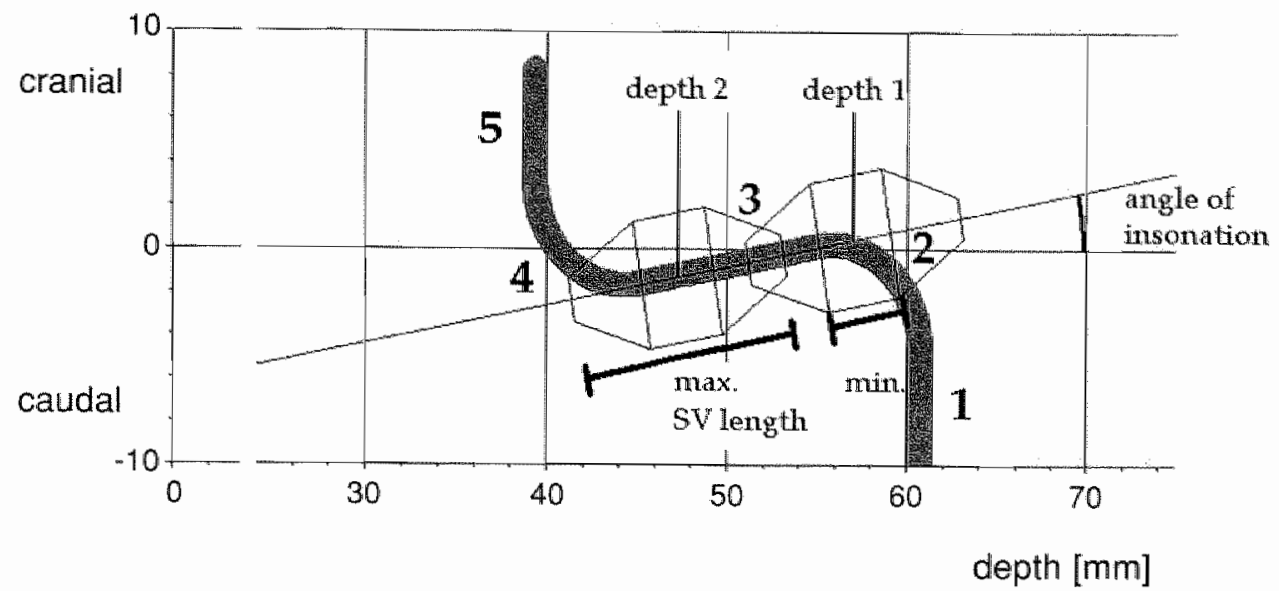

Filigure $1 \mathrm{a}$

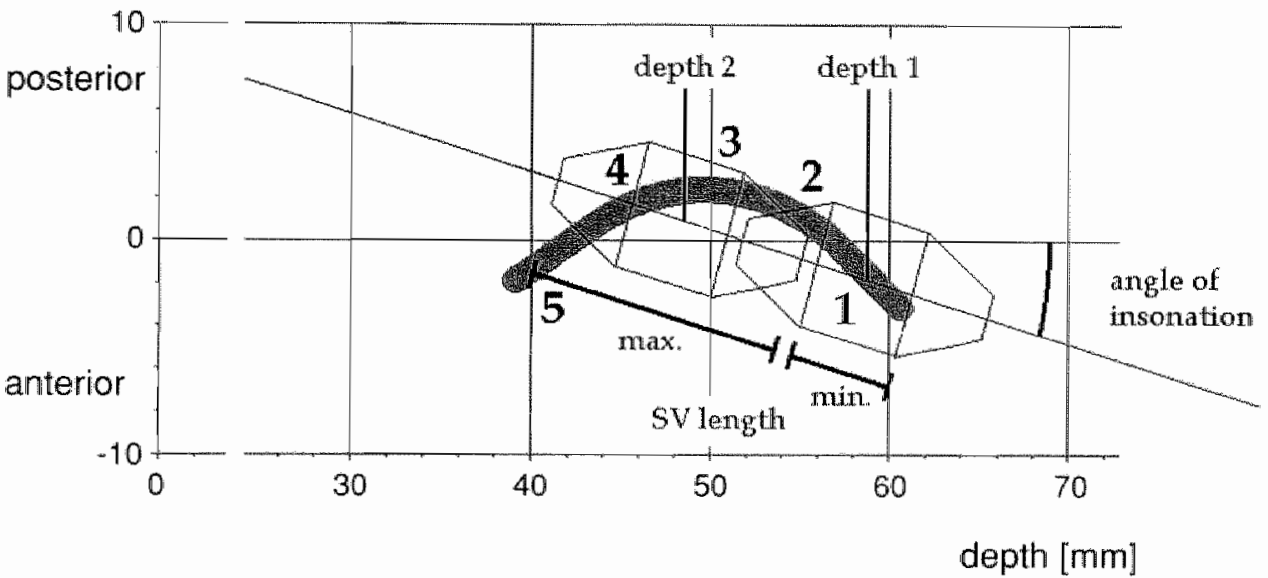

\section{Figure $1 a$ and $b$}

Simulatted vessel in the (a) Coronal and (b) Axial pHane. The graphical display shows the first vessel simulated in the cononal and axial planes. The wessel consisted of three stratght parts $(1,3,5)$ at the proximal end the distal and and in the anddle, which were adjustable for length. Two curwed parts $(2,4)$ were added in between, which could be adjusted for angle andi radius (dteternining how curved the vessel was and how long this curved part wasi. Additionally, the position of the vessel relatied to the skull could be changed. The insonation angle and the sample volume burst length, as well as the pulse repetition frequency (PRF), couid be changed for both sample volumes. Each sample volume could be independently adjusted for receiver gatte time (RG) and depth. The shortest and longest possible sample voltume lengths, depending on the RG setting ane indicated. 
transcranially monitoring patients with atherosclerosis, these factors are likely to be of importance. So, combining the indicated path and velocity of the MES with the sensitivity profile of the sample volume (isoamplitude mapping) and a choosen sampling frequency (PRF $4170 \mathrm{~Hz}$ ), resulted in an MES without any background and with clearly defined start and end points.

In a second step, we added random noise with half the amplitude of the MES (e.g, if the MES reached a maximum amplitude of 0.7 , then the random noise level was set to 0.35 , indicating an MES: blood ratio of $6 \mathrm{~dB}$ ). This refers to the concept of the embolus to blood ratio as proposed by Moehring and Klepper (1994). Due to our experience with manual measurements of start and end points of MESs in real-time domain data (Mess et al. 1996), an amplitude difference of $6 \mathrm{~dB}$ between MES and background is necessary to be able to perform reliable measurements. The theoretical start point of the MES was then calculated on the basis of the abovementioned alssumptions (i.e., there was no algorithm or specific measurement tool used for the determination of the time characteristics of the MES). This approach, thus, does not take into account the influence of various types of MES measurement techniques, but gives the best possible estimation of the "measurable start point" of an MES, independent of measurement techniques.

\section{The tests}

Altogether, eight studies were performed with three different vessel anatomies. Table 1 gives an overview of the various TCD variables used in our study.

\section{Table 1. Overview of the studies}

\begin{tabular}{|c|c|c|c|c|c|c|c|c|}
\hline & anatomy & $\begin{array}{l}\text { sample vol. } 1 \\
\text { clepth Imm }\end{array}$ & burst-length |fis] & RG [u:s] & $\begin{array}{l}\text { sample vol. } 2 \\
\text { depth [mm] }\end{array}$ & burstlength [us] & $\mathbb{R G}[\mu, 5]$ & $\begin{array}{l}\text { inson. } \\
\left.\text { angle }{ }^{\circ}\right]\end{array}$ \\
\hline Study I: & vessel i & 60 & 13 & 6 & 50 & 13 & 6 & $-5 \cdot 25$ \\
\hline Situdy I & vessiel l & $55-64$ & 13 & 6 & $45-54$ & 13 & $E$ & 15 \\
\hline Situdy III & vessel | & 58 & 10 & 6 & 54 & 10 & 2 & $-5 \cdot 25$ \\
\hline Study IV & vessiel \# & $49-58$ & 10 & 6 & $45 \cdot 54$ & 10 & 2 & 15 \\
\hline Study W & versel 2 & $55-64$ & 13 & 6 & $45 \cdot 54$ & 13 & 6 & 15 \\
\hline Study WI & vessel 2 & $51-58$ & 10 & $B$ & $47-54$ & 10 & 2 & 15 \\
\hline Study VII & vessel 3 & $55-64$ & 13 & 6 & $45-5.4$ & 13 & 6 & 15 \\
\hline Study VIII & vessel 3 & $50-58$ & 10 & 6 & $46-54$ & 10 & 2 & 15 \\
\hline
\end{tabular}

The vessel numbers refer to the explanation in the text.

First, a "mean MCA" was simulated according to data from anatomical studies (Jain 1964; Lang et al. 1979). The vessel had a mainstem length of $20 \mathrm{~mm}$, a dorsal convex curve in the axial plane, and an oblique descending and downward convex course in the coronall plane (Vessel 1). Four simulation studies (I-IV) with different TCD settings were performed with these vessel settings. 


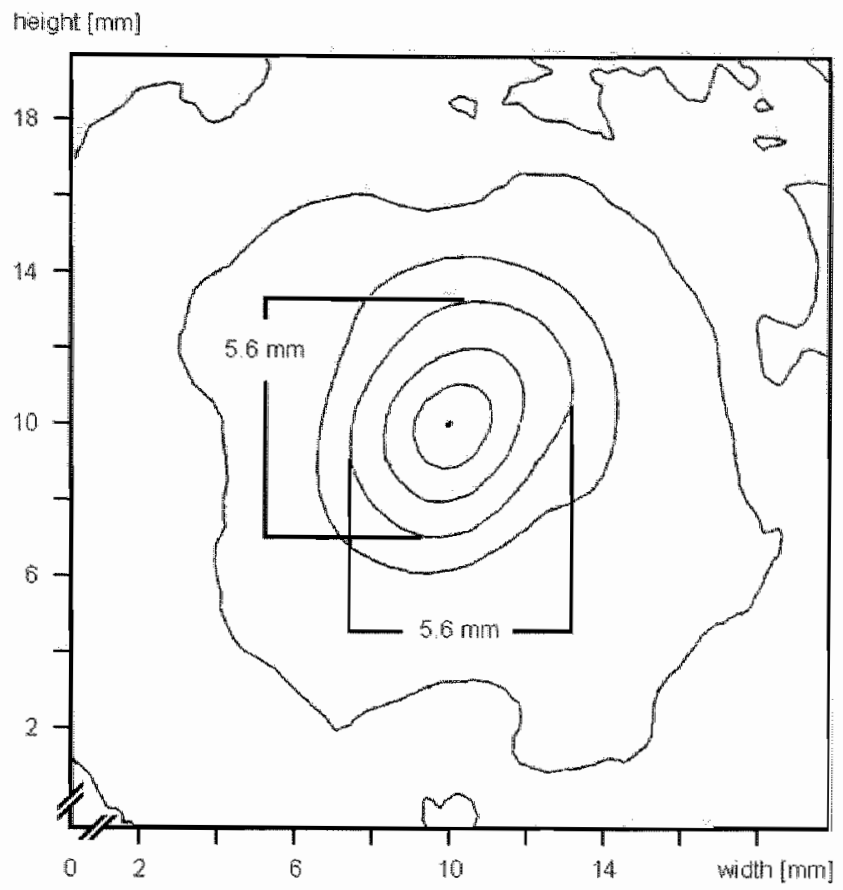

Figure 2a

width [mm]

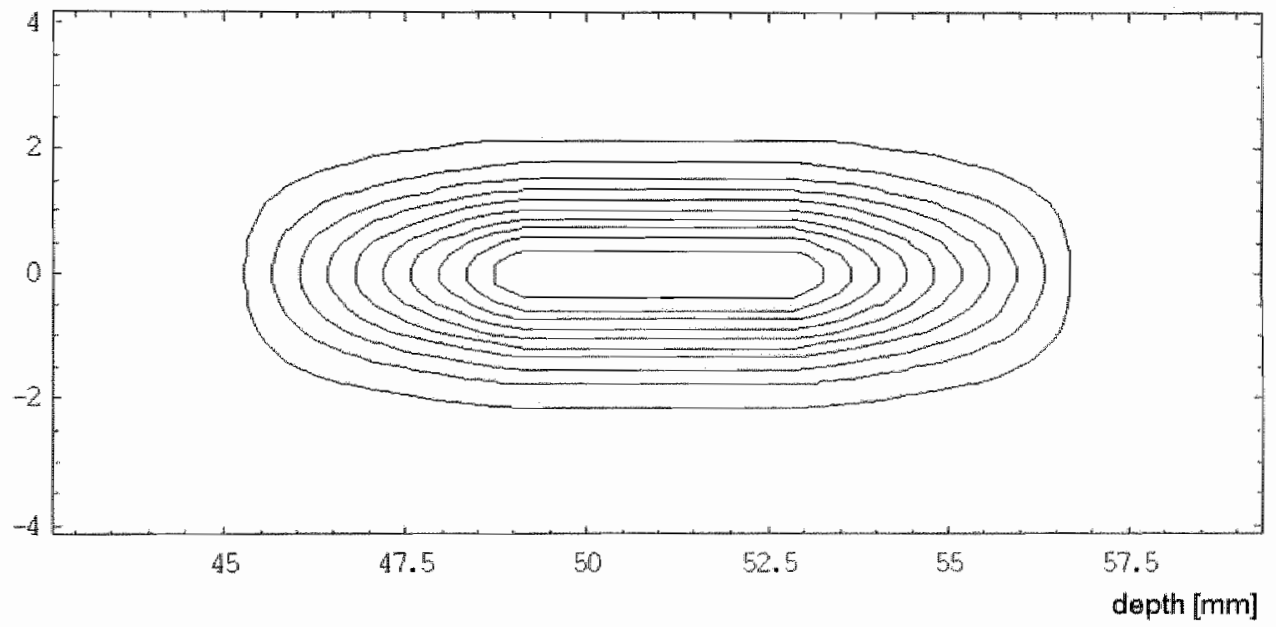

Figure $2 a$

Lateral acoustic pressure piot of the 2-Mry probe used as the model for the computer simulation, The graph shows the dectine of the lateral sensitivity at a depth of 4.1 mum (i.e, at maximum axial sensitivity), Each circular Ine represents a decrease of 1 dB as compared to the inner circle, (Data provided by EME, Klenostheim, Gernany; results of waterbath experiment with a hydtophon.)

\section{Figure $2 \mathbf{b}$}

Analytical fit of amplitude mapping. This is an example of an analyntical fit for a burgt length of 10,4 and a receiver gate time of is $u s$ as used for the proximal sample volume in the settings. The center represents the area that whill produce the maximum amplitude of 1 if an MES passes this region. Each lime outwards represents a decrease of $10 \%$ of amplit wide. The $x$ axis incticates the depth, the $y$ axis the widith of the sample volume. 
The influence of different angles in the axial plane (Study l) (Filg. 3a) and those of changing depth values (Study II) (Fig. 3b) were selectively evaluated, keeping all other parameters unchanged. Initially, the sample volume burst length was 13 us (which equals about $10 \mathrm{~mm}$ ) with a gate separation of $10 \mathrm{~mm}$ between the centers of the sample volumes. The receiver gate (RG) time was set to $6 \mu$ s. So, the maximall sample volume length, independent from sensitivity distribution, was about $20 \mathrm{~mm}$. With respect to the distance between the sample volumes, we called these the "10-settings."

It seems reasonable to assume that, if the sample volumes are shorter and closer together, the range of the $\Delta t$ values will become smaller. However, due to our main area of interest, which is perioperative monitoring during carotid endarterectamy, it is very difficult to routinely use a sample volume burst length significantly shorter than $13 \mu$ s (equaling $10 \mathrm{~mm}$ ). This often will result in an unstable envelope because less backscatter signall will be available to analyze the maximum Doppler shift. Evaluation of hemodymamic parameters, which are necessary for guiding the surgeon with respect to the use of a shunt, will then become impossible.

Therefore, we tested a second TCD setting that consisted of slightly shorter sample volume bursts ( 10 us equaling $8 \mathrm{~mm}$ ), but a considerably shorter gate separation $(4 \mathrm{~mm})$. Additionall $\mathrm{y}_{\mathrm{u}}$ the $\mathrm{RG}$ time in sample wolume 2 (more distall) was set to $2 \mu s$, resulting in a relatively short maximal sample volume length (about $11 \mathrm{~mm}$ ). We called this the "4msettings." It is important to realize that the length of the RG is mainly responsible for the length of the less sensitive parts at the ends of the sample volume in the axial direction (see Fig. 1 and Fig. 2). This effect allows a strong backscatterer (e.g. an MES of high intensity) to appear longer in the sample volume than a weak backscatterer. However, the shorter the RG time, the weaker the total backscattered signal that can be further analyzed. The proximal sample volume (sample volume 1) could, thus, theoretically be used for the hemodynamic evaluation, the distal one for the $\Delta t$ calculations. The Studies I and II were then repeated with these settings (i.e., Studies III and IV).

Second, the angle of the MCA mainstem in the coronal plane was changed. Instead of the downward course of Vessel $1\left(-10^{\circ}\right)$, the angle was upward $\left(20^{\circ}\right)$, resulting in a steeper angle between insonation axis and vessel (Vessel 2). The effect of changing the insonation dlepth was examined, applying both TCD settings (Studies $V$ and VI).

Third, the vessel described for Studies IIV was shortened (i.e., the mainstem length decreased to $14 \mathrm{~mm}$ ) (Vessel 3). Again the effect of changing the insonation depth was examined applying both TCD settings (Studies VII and VIII).

Every simulation consisted first of MES measurements without background signal. As described above background noise was introduced with half the amplitude of the maximum amplitude of the MES. The beginning and the length of each MES (i.e., the part reaching beyond background) were measured, which allowed additionally to calculate the $\Delta t$ value. 


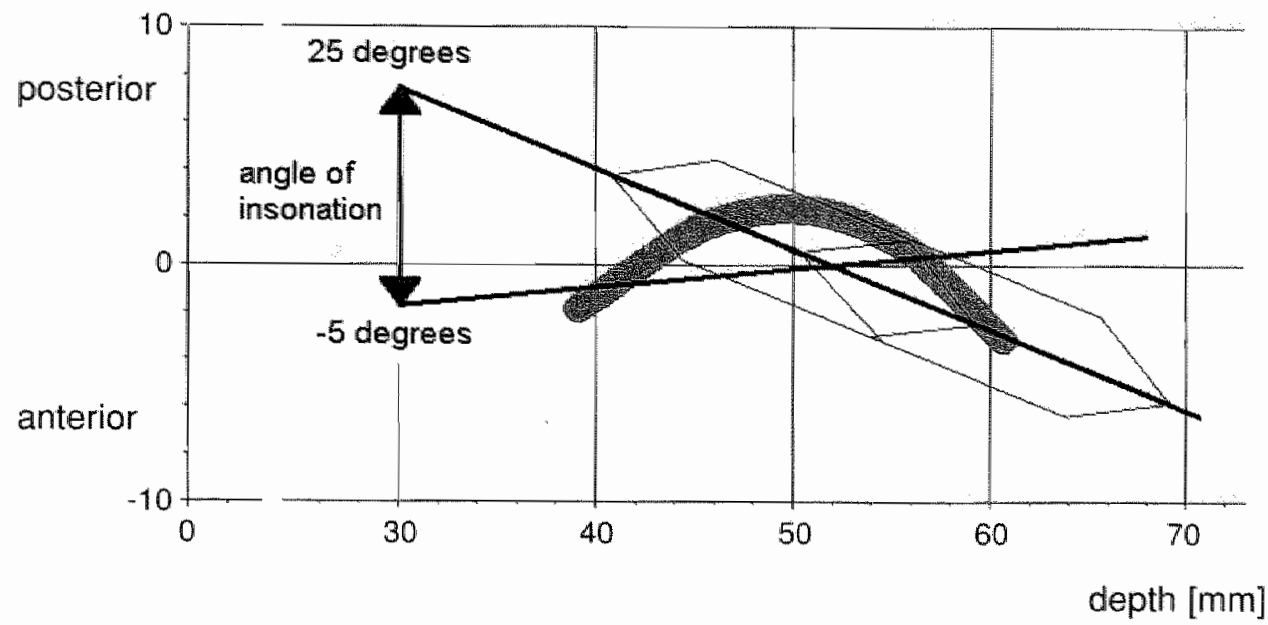

Figure $3 a$

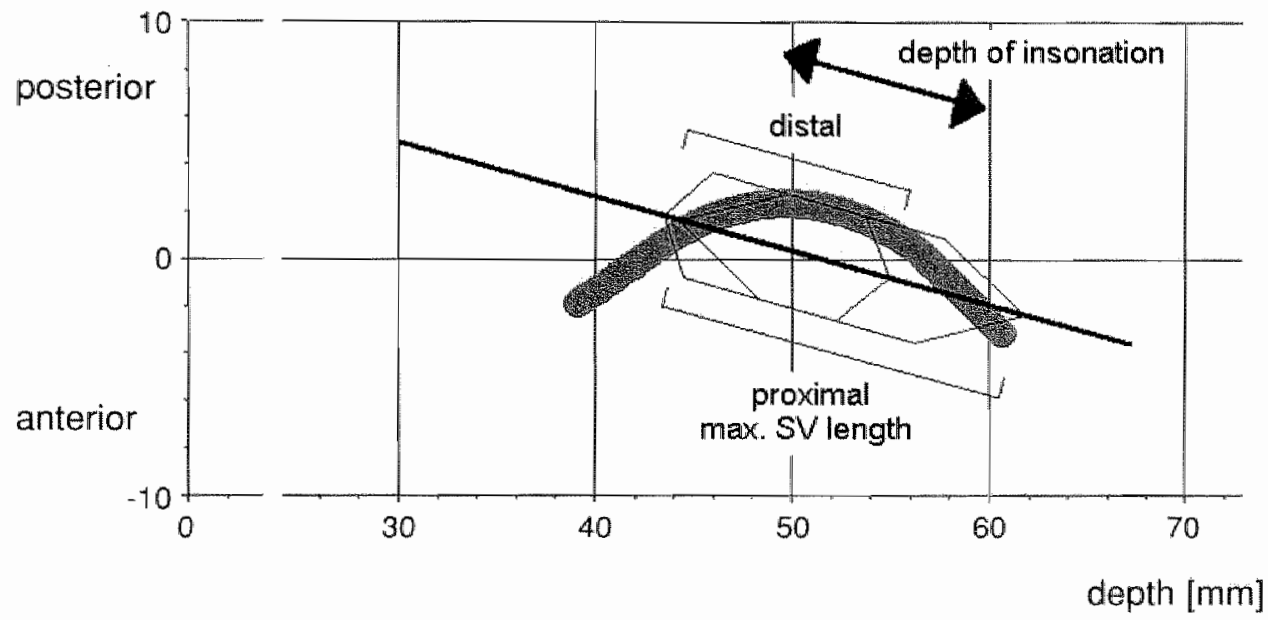

\section{Figure $3 a$}

Changing the insonation angle in the axial plane (Studies a and thl. For the studies evaluating the effects of different insonation angles, the insonation clepth of the sample volumes remainad unchanged. This example shows how the insonation axis in the axkial plane was changed for Study 1 (burst length $13 \mu$ s; receiver gate 6 , 5 ; insonation at a depth of 50 and $60 \mathrm{~mm}$ ).

\section{Figure 3a}

Charging the insonation depth (Sitdies ll and W to Vill). For the studies evaluating the effects of different insonation depths, the sample volumes were shifted along an unchanged insonation axis with an insonation angle of 15 . The figuren wisplays an examplis

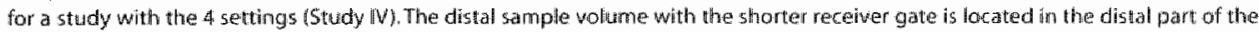
larger proximal sample volume; the gate separation is 4 namt. 
ReSUltS The results are presented in two groups: first, data from measurements of the duration of the MES are shown. Then, the $\Delta t$ values will be dealt with.

\section{Duration of MES}

We restricted this part of the study to the first vessel configuration (Vessel 1) and the 10 settings (Studies I and II). The impact of the angle of insonation on the MES length was tested proximally (th; sample volume depth $60 \mathrm{~mm}$ ) and distally ( $t_{2}$; sample volume depth $50 \mathrm{~mm}$ ).

Figure $4 a$ demonstrates that, for both sample volumes, $t_{1}$ and $t_{2}$ are quite variable. This variation is dependent on the angle of insonation as well as on the EBR value.

Figure $4 \mathrm{~b}$ shows comparable results. Using an angle of insonation of $15^{\circ}$ and changing the depth of the sample volume in $1-\mathrm{mm}$ steps from 45 to $65 \mathrm{~mm}$ results in a significant increase of the variation of $t_{1}$ and $t_{2}$, which is also dependent on the EBR value.

\section{$\triangle \uparrow$ values of $M E S$}

The $\Delta t$ values of the simulation tests with the first vessel are given in Fig. $5 \mathrm{a}$ and $\mathrm{b}$. Tilting the insonation axis in the axial plane (Fig. $5 \mathrm{a}$ ) resulted in $\triangle \mathrm{t}$ values from $8 \mathrm{~ms}$ to $26 \mathrm{~ms}$ for the MES simulated with the 10 settings (Study I), and from $3 \mathrm{~ms}$ to $19 \mathrm{~ms}$ for the MES simulated with the 4 settings (Study III), depending mainly on the angle, but also on the EBR value. So, the variation of the $\Delta t$ values was comparable for both Doppler settings. The difference between the two settings in terms of the $\Delta t$ range ( 4 settings produced shorter $\Delta t$ values) fairly corresponds with the smaller gate separation of the latter Doppler settings.

However, in terms of variability, the two settings showed a significant difference, when the sample volume depth was changed (Fig. 5b). The 10 settings (Study II) resulted in $\Delta t$ values that ranged from 3 to $31 \mathrm{~ms}$. In contrast, the 4 settings (5tudy IV) resulted in $\Delta t$ values that ranged from 8 to $14 \mathrm{~ms}$. The influence of gradually increasing the sample volume dlepth when simulating a steeper angle between insonation axis and MCA mainstem (Vessel 2 ; Studies $V$ and VI) or a shorter MCA mainstem (Vessel 3; Studies VII and VIII) is shown in Fig. $5 \mathrm{c}$ and $\mathrm{d}$. With Vessel 2 , the range of the $\Delta t$ is still smaller for the 4 settings, especially for high intensity MES (6-14 ms vs. 1-23 ms with 10 settings). Applying Vessel 3 , the advantage of these settings becomes even more obvious.

The last example (Vessel 3 ; Fig. 5 d) demonstrates best how the 10 settings can fail in terms of $\Delta t$ walues. Though the MES was present in the Doppler signal until a depth of $54 \mathrm{~mm}$ (and so the examiner can hear it\}, the $\Delta t$ values obtained would only be useful in terms of applicability for an automated system until a depth of $50 \mathrm{~mm}$. With an insonation depth of $51 \mathrm{~mm}$ or more, the resulting $\Delta t$ values were $3 \mathrm{~ms}$ or shorter. These values are likely to be inadequate for a reliable automated measurement of the time difference. Between $45-$ and $50-\mathrm{mm}$ depth, the $\Delta t$ walues ranged from $3 \mathrm{~ms}$ to $19 \mathrm{~ms}_{\text {, }}$ depending also on the EBR. When applying the 4 settings, the depth range that produced useful $\Delta$ t values could be enhanced and the variability was also considerably lower ( 7 to $14 \mathrm{~ms}$ between 46 and $50 \mathrm{~mm}$ ) compared to the 10 settings. 


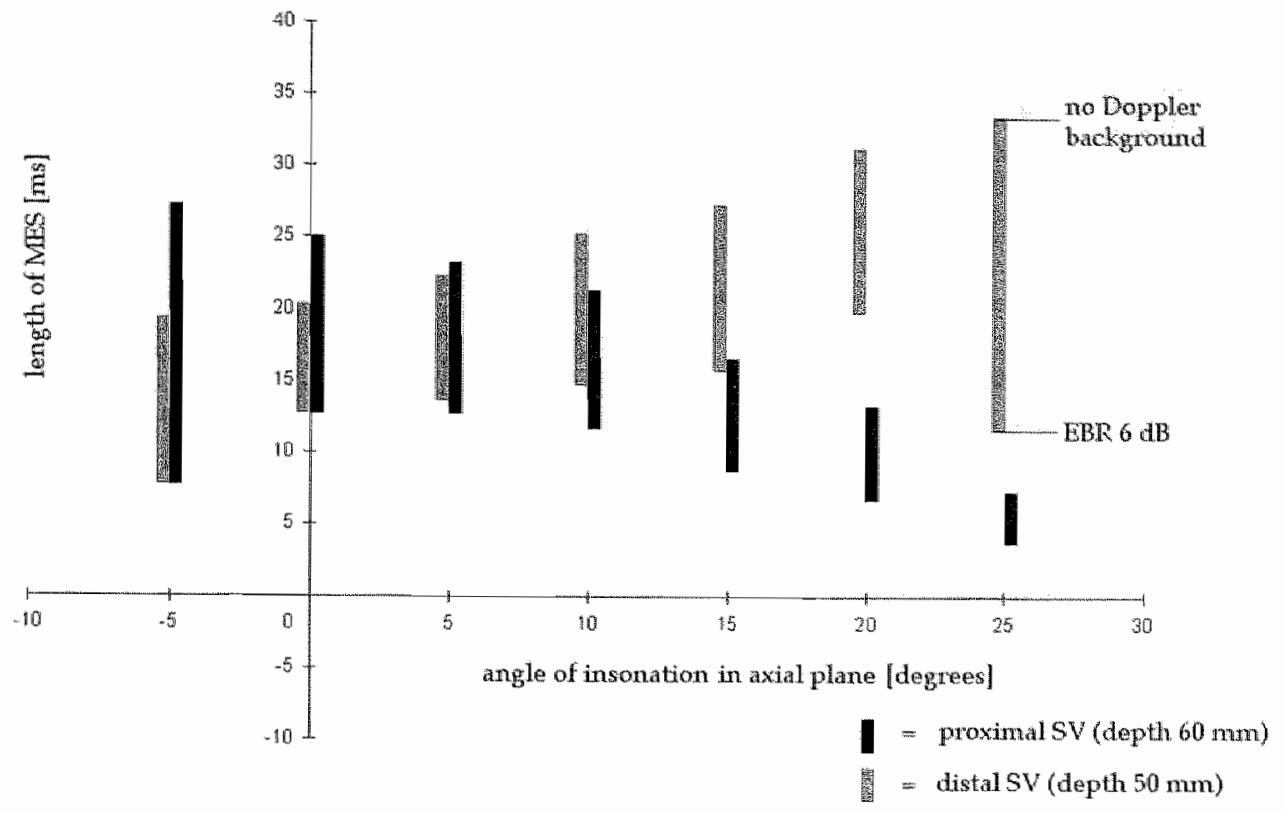

Figure $4 a$

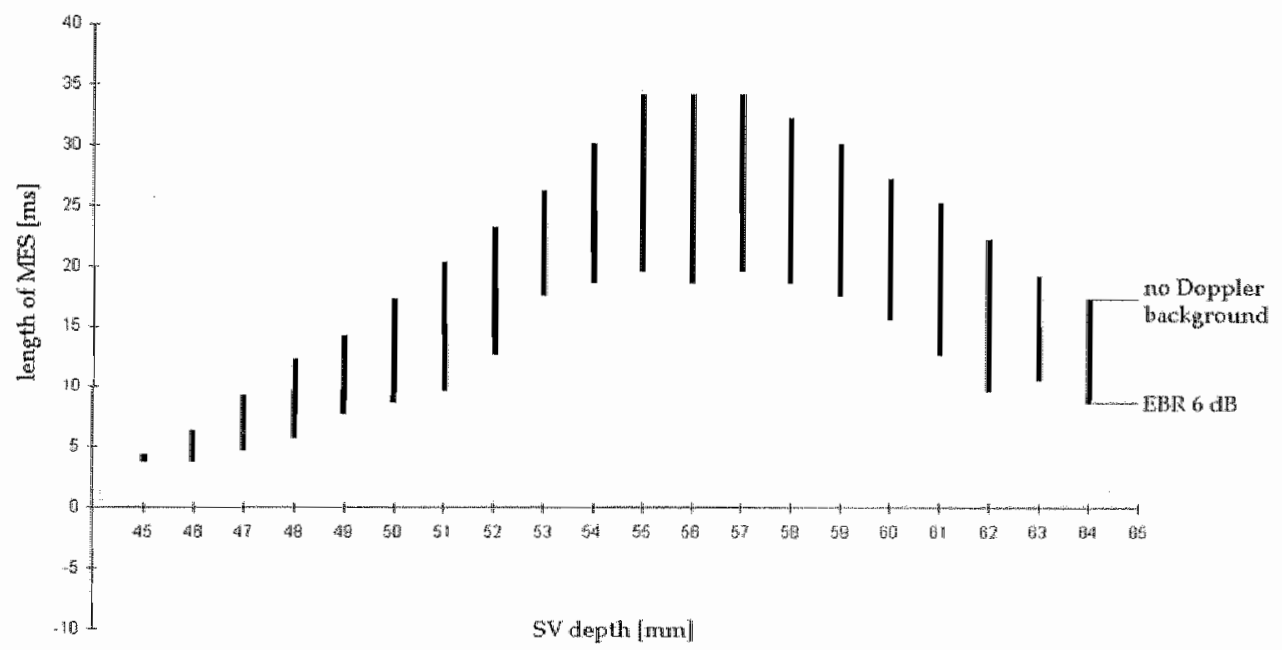

Figure 4a

Length of MES with different insonation angles at two different depths. The bars indicate the wariation of MEs lemghth values measulfed in ms (y axis) for different anglew $(x$ axiss) at (wo different depths of insonation (grey bars $=50 \mathrm{~mm}$ and black bars $2 x$ $60 \mathrm{~mm}$ ). The maximum walue was achiewed when no Doppler background was applied, the minimurm walue will an MES/background ratio of 6 r

\section{Figure $4 b$}

Length of MES wh different insonation depths. The bars indicate the wariation of MES length wallues meastured in: ms (y axis) for different depths of insonation. Agarn, the highest walues were recognized when no Doppler background was applied, the lowest values with the smallest MES intensity possible: 16 dB: 


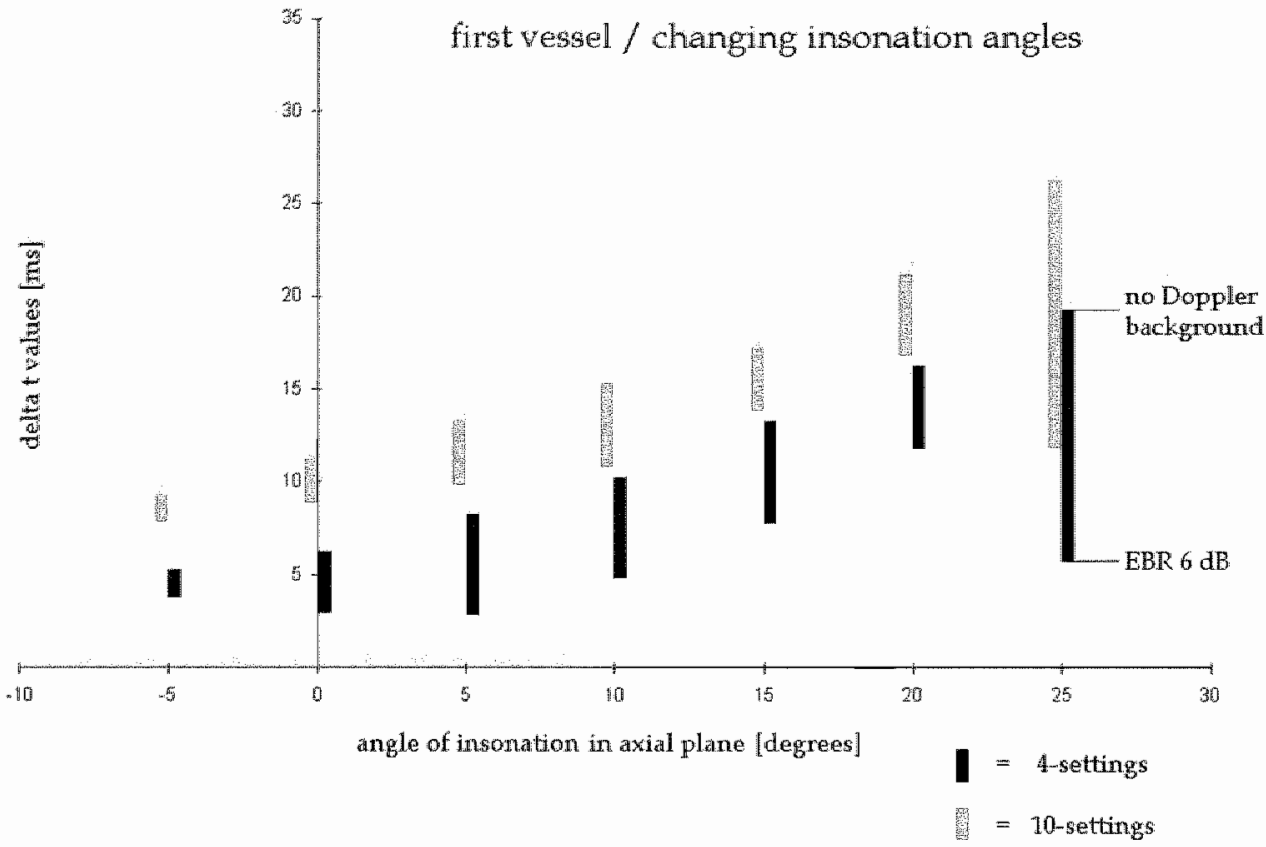

b

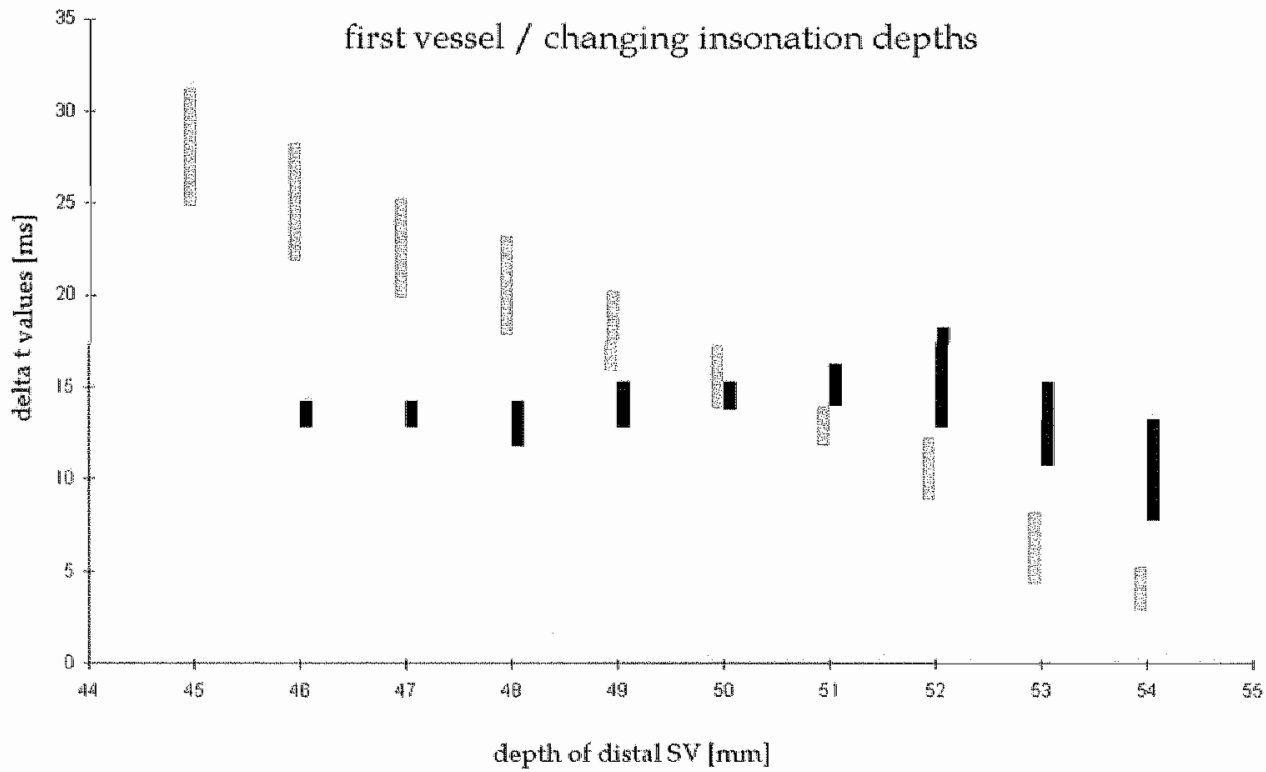

Figure 5 a and b

Dettat Talues. The At values of the MES when entering the sample volumes are displayed on the $y$ axis expressed in ms. The gray bars show the possible ange of the At values for the 10 settings, the black bars thase of the 4 settings. Similarty to the study on MES length, the highest wafles were obtained when no Doppler background was applied, the lowest values with an MESibackground ratio of $6 \mathrm{~dB}$. (al The affect of changing twe insonation axis in 5 degree steps ( $x$ axis) on MEs length is shown as the first vessel. The effect of changing the insonation depth in 1-mum steps. ( $x$ axis) on MES length is shown for (b) the first, (c) second and ld) third vessel. 


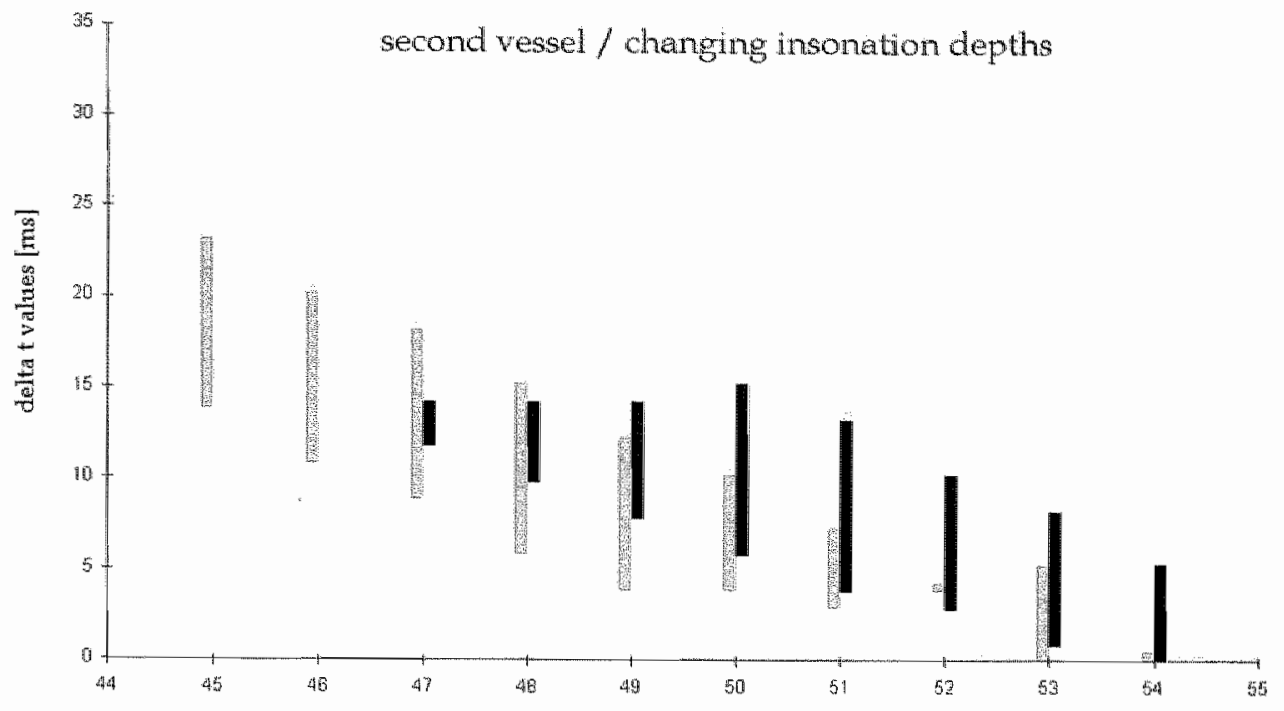

depth of distal SV [mm]

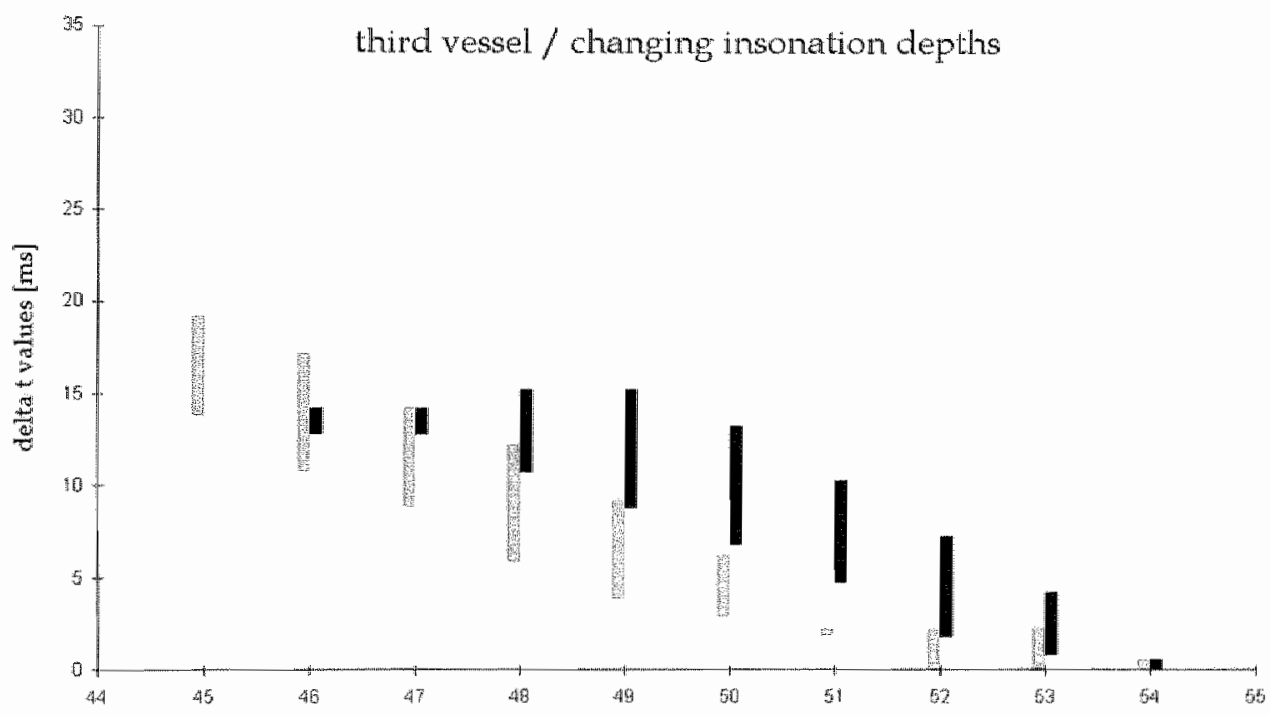

Figure $5 i c+d$

Delta $T$ values. The $\triangle t$ walues of the MES when entering the sample wolumes are displayed on the y axis experssed in ms. The gray bars show the pos sible range of the Az walues for the 10 settingls, the black bars those of the of settings. Simbarly to the study on Mtes length, the highest walues were abtained when no Doppler background was applied the lowest wallues with an MES/twackgnand ato of $6 \mathrm{~dB}$. (a) The effect of thanging the insonation axis in 5 degree steps $(x$ axis) on MES length is shown as the first vessel The effect of changing the insonation depth in 1 -inm steps (x axis) on MES length is showm (for (b) the first, (c) second and (d) third wessel. 
However, the simulations with Vessels 2 and 3 demonstrate that, with very low MES intensity (EBR $6 \mathrm{~dB}$ ), the 4 settings can also produce rather short $\triangle 4$ values, if the MCA mainstem is covered by the ultrasound beam only over a short distance, due to an unfavorable insonation angle (Vessel 2) or a short MCA mainstem (Vessel 3). 
DisCUSSiOn At this moment, there is an increasing interest in long-term MES detection in several areas of cerebrovascular care and reseanch. However not patients with an MES positive carotid artery stenosis could profit from carotid endarterectomy; will remain unsolved until large-scale prospective examinations are done (Siebler et al. 1995; Georgiadis et all. 1997). So, there is a considerable need for an automated emboli detection that can reliably replace the human observer. Additionally, an automatic system bears the chance of avoiding interobserver variability with MES of low intensity (Markus et al. 1997). In a recent study (wan Zuilen et al.1996), our group could show that supervision of TCD monitoring for MES by experienced human observers was still necessary when compared with three commercially available systems that automatically detected MES.

If the dual-gate technique, as a potential candidate for improving the performance of computed systems, is applied in an automated algorithm, it is crucial that the $\Delta t$ between the MES in the two sample volumes be reliably measured. "Missing emboli" as discussed by Smith et al. (1997a), and a large range of the $\Delta t$ values are likely to complicate an automated signal analysis. Droste and colleagues (1997) tested an MES detection software that applies the dual-gate technique as an essentiall part of the detection algorithm. Their reported sensitivity values for detecting MES were comparable with those of our own study (van Zuilen et all. 1996), based on the singlegate technique. Interestingly, their data also showed a wide range for the time lag between the two MES. The presented simulation model confirms the critical role of the MCA anatomy for the MES characteristics in TCD. We showed a remarkable variation of the length of single MES when applying standard TCD settings, and our first vessel that was configured according to mean anatomical properties, as reported from autopsy and angiography studies. The data were in good agreement with our patient data (Mess et al. 1996) (i.e., the values for MES length were in a comparable range). Due to the experimentall setup, it could be shown that changing the depth and also the angle of insonation caused largely different MES duration values. Particularly, the more proximal and distal sites of insonation evoked considerably shorter values for MES length compared to a sample volume placement in the middle of the MCA. At these locations, the MES entered or left the sample volumes laterally instead of the front or rear end. Comparable effects were seen when angulating the insonation axis, which confirms the data of Simith et al. (1997a), who also could demonstrate a dependency of MES length and $\Delta t$ in a computer model that simulated a dual-gated TCD. The fast lateral sensitivity decay of the sample volume is responsible for that phenomenon. The lateral sensitivity characteristics we applied in our model are in good agreement with data from Arnolds et al. (1989), who used a probe from the same company in a waterbath experiment to measure the axial and lateral sensitivity.

A correlation could also be demonstrated between the intensity of the MES and the length of the signal in the simulated raw data. These data indicate first, that the calculation of MES strength using the length of the signal in the time domain (the "effective sample volume," as proposed by Simith et al. in 1995) largely depends on the location of the sample volume relative to the vessel segment insonated. The possible range for the length values of MES with different EBRs can wary significantly. 
This range is relatively simall when the sample volume is located at the proximal ar distal end of the vessel. This effect could be due to the MES entering or leaving the sample volume from the side, thus "bypassing" the gradually less sensitive regions of the sample volume at the front or rear end. The ability to grade the MES intensity using the length of the signal in the raw data depends on how the MES passes through the sample volume. The sensitivity increase at the side of the sample wolume is wery steep (data from EME, Smith et al. 1997a) and allows considerably less differentiation. lin practical terms, the interaction between sample volume sensitivity and MES length renders judgement of the MES intensity if defined as effective sample volume length difficult and is likely to add to the large intensity ranges of both gaseous and particulate MES, which have been reported by 5 mith et al. (1996).

Second, the likelihood of getting different length information of one MES increases using the dualgate technique with usual settings, because the technique asks for placing at least one sample volume at the proximal or distal end of the MCA.

We did not test for missing emboli because the structure of the model easily allowed creation of the situation where an MES only is present in one of the sample volumes. 5o, this question could not be answered without considerable bias.

Tilting the insonation axis in the axial plane and, even more, shifting the sample volume depth on the insonation axis resulted in a striking variation of the $\Delta t$ values at the proximal site of the sample volumes, when a mean anatomic vessel was simulated with usual TCD settings. Similarly to the length measurements, the $\Delta t$ values varied with different EBR, values, but the position of the sample voliume relative to the vessel was far more important for the calculation of the values. The importance of the vessel anatomy became accentuated when a critical vessel with a shorter mainstem and a larger angle with the insonation axis was mimicked. Though a Dopplier signal was defined over a range of $10 \mathrm{~mm}$ for the distal sample wolume, the $\Delta t$ values obtained were nat suitable for a reliable calculation when the sample volume was placed in the proximal half of the abovementioned range.

From the study on the MES length, it seemed reasonable in terms of lowering the remarkable variation of $\Delta t$ to make the sample volumes shorter and put them closer together. However, during carotid endarterectomy, at least one Doppler channel has to provide sufficient hemadynamilc data. Therefore, we choose an adequate sample volume burst length of $10 \mu 5$ and a RG of $6 \mu$ s for the sample volume in the middle of the vessel, providing a sufficient $S: N$ ratio. Despite a small gate separation of $4 \mathrm{~mm}$, we noticed $\Delta t$ values comparable to those obtained with the 10 settings. This was achieved by means of the short receiver gate time (2 $\mu$ s instead of $6 \mu 5)$ in the distal sample volume. With the "mean anatomic vessel, "a distinct positive effect in terms of variability could be shown. However, the advantages when mimicking a more difficult vessel were present but less impressive.

In conclusion, our simulation showed that with anatomically realistic vessels, calculations on time characteristics of MESs show extreme variations, with only modest changes of the insonation angle or the sample volume depth. Monitoring thuman vessels in wivo will add even more variability than 
we simulated because, first, we did not apply a pulsatile background with its changing backscatter intensities (see e.g., Markus and Molloy 1997a); second, theire were no small wessels branching off: and third, the complex distribution of flow velocity profiles in curved vessels as show by Jou et al. (1996) were not incorporated into the model. The latter implies that MES could not switch between the different velocity layers of the vessel, as proposed by 5 mith et al. (1997b) as an explanation for MES with changing velocities. These results not only urge us to a cautious interpretation of the properties of single MES, but also contribute to an understanding of the marked $\triangle t$ variatioin using the dual-gate technique. Actually, TCD applying the dual-gate technique for MiES detection is confronted with a black box system of extremely high variability with the MCA anatomy playing a major role. Despite the fact that the examiner gets a Doppler signal, the resulting $\Delta t$ values seem to be unforeseeable. Only the TCD settings can be adjusted in this system. It could be shown in the present study that, with an appropriate choice of these settings (i.e. a relatively narrow gate separation and a short RG of the distal sample volume), the expected $\Delta t$ values are in a comparatively narrow range, making them more suitable for an automated approach. 


\section{References}

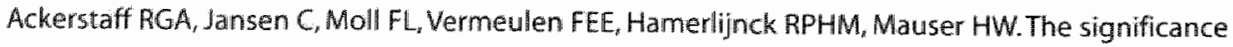
of microemboli detection by means of transcranial Doppler ultrasonography monitoring in carotid endarterectomy. J Vasc Surg 1995;21:963-969.

Arnolds BJ. Kunz D, von Reutern GM. Spatial resolution of transcranial pulsed Doppler technique in vitro evaluation of the sensitivity distribution of the sample volume. Ultrasound Med Biol 1989; 15:729-735.

Droste DW, Markus HS, Brown MM. The effect of different settings of ultrasound pulse amplitude, gain and sample volume on the appearence of emboli studied in a transcranial Doppler model. Cerebrowasc Dis 1994a; 4:152*156.

Droste DW, Markus HS, Nassiri D, Brown MM. The effect of velocity on the appearence of embolic signals studied in transcraniall Doppler models. Stroke 1994b;25:986-991.

Droste DW, Hagedorn G, Nötzold A, Siemens HJ, Sievers HH, Kaps M. Bigated transcranial Doppler for the detection of clinically silent circulating emboli in normal persons and patients with prosthetic heart valves. Stroke $1997 ; 28: 588-592$.

Gaunt ME, Martin PJ, Smith JL, Rimmer T, Cherryman G, Ratliff DA, Bell PRF, Naylor AR.

Clinical relevance of intraoperatiwe embolization detected by transcranlal Doppler ultrasonography during carotid endarterectomy: a prospective study of 100 patients. Br J Surg 1994;81:1435-1439.

Georgiadis D, Goeke J, Hill M, König M, Nabovi DG, Stögbauer F, Zunker P, Ringelstein EB. A novel technique for identification of Doppler microembolic signals based on the coincidence

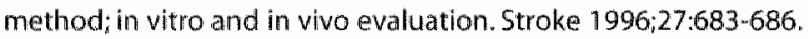

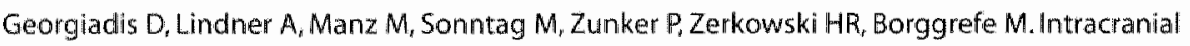
microembolic signals in 500 patients with potential cardiac or carotid embolic source and in normal controls. 5troke 1997;28;1203-1207.

Huber $P_{\sharp}$ Krayenbühl H, Yasargil G. Zerebrale Angiographie für Klinik und Praxis. Stuttgart: Theme Verlag, 1979.

Jain KK. Some observations on the anatomy of the middle cerebral artery. Can J Surg 1964;7:134-139. 
Jou LD, van Tyen R, Berger SA, Saloner D. Calculation of the magnetization distribution for fluid flow in curved vessels. Magn Res Med 1996:4,577-584.

Lang J, Bushe KA, Buschmann W, Linnert D. Kopf, Teil B, Im: von Lanz T, Wachsmuth W, ed. Praktische Anatomie. Ein Lehr- und Hilfsbuch der anatomischen Grundlagen ärztlichen Handeins. Berlin: Springer, 1979.

Levi CR, O'Malley HM, Fell G, Roberts AK, Hoare MC, Royle JP, Chan A, Beiles BC, Chambers BR, Bladin $C F$, Doman GA. Transcranial Doppler detected cerebral microembolism following carotid endarterectomy; high microembolic signal loads predict postoperative cerebral ischaemia. Brain 1997; 120:621-629.

Markus HS, Molloy J. Use of a decibel threshold in detecting Doppler embolic signals. Stroke $1997 \mathrm{a} ; 28: 692-695$.

Markus HS, Ackerstaff RGA, Babikian VL, Bladin CF, Droste DW, Grosset DG, Levi CR, Russell D, Siebler $\mathrm{M}_{\text {* Tegeler }} \mathrm{C}$. Intercenter agreement in reading Doppler embolic signals; a multicenter international study. Stroke 1997b;28:1307-1310.

Mess WH, Titulaer BM, Ackerstaff RGA. An in vivo model to detect microemboli with multidepth technique. Preliminary results (abstract). Cerebrovasc Dis 1996;6;5uppl.3:60.

Moehring MA, Klepper JR. Pulse Doppler ultrasound detection, characterization and size estimation of emboli in flowing blood. IEEE Trans Biomed Eng 1994;41:35-44.

Siebler M, Nachtmann A, Sitzer M, Rose G, Kleinschmidt A, Rademacher J, Steinmetz H. Cerebral microembolism and the risk of ischemia in asymptomatic high-grade internal carotid artery stenosis. Stroke 1995;26:2184-2186.

Smith JL, Evans DH, Fan L, Gaunt ME, London NJM, Bell PRF, Naylor AR. Interpretation of embolic phenomena during carotid endarterectomy. Stroke1995;26:2281-2284.

Smith J ${ }_{1}$ Evans DH, Fan L, Bell PRF, Naylor AR. Differentiation between emboli and artefacts using dual-gated transcranial Doppler ultrasound. Ultrasound Med Biol 1996;22:1031-1036.

Smith JL, Evans DH, Naylor AR. Signals from dual gated TCD systems: curious observations and possible explanations. Ultrasound Med Biol 1997a;23:15-24. 
Smith LL, Evans DH, Naylor AR. Analysis of the frequency modulation present in Doppler ultrasound signals may allow differentiation between particulate and gaseous cerebral emboli. Ultrasound Med Biol 1997b;23:727-734.

vain Zuilen EV, Mess WH, Jansen C, van der Tweel I, van Gijn J, Ackerstaff RGA. Automatic embolus detection compared with human experts; a Doppler ultrasound study. Stroke 1996:27:1840-1843. 
4

A new algorithm for off-line automated emboli detection based on the pseudo Wigner power distribution and the dual gate TCD technique Ultrasound Med Biol 2000;26:413-418 


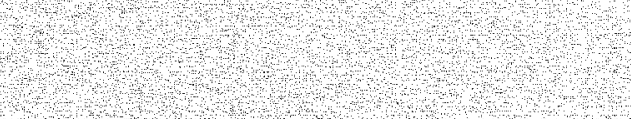


Abstract Research on microembolic signals (MES) using the dual gate technique has shown promising results, when the time difference $(\Delta t)$ of an MES in two sample volumes (SVs) placed serially has been measured manually. On the other hand, the computerized discrimination of MES and artefacts has been reported not to be superior to algorithms based on a single SVITherefore, a dataset containing MES as well as four types of artefacts was made to test a preliminary version of a new algorithm for automated embolli detection. We monitored 20 patients during carrotid endarterectomy $(n=17)$ and heart surgery $(n=3)$. Two transcranial Doppler (TCD) signals with a partial overlap of the SVS were recorded online and analysed off-line with an algorithm based on three consecutive steps: 1 . Is there an intensity increase in both channels (64-point $\mathrm{FF}$; $50 \%$ overlap)? 2. What is the expected $\Delta t$, with the velocity measured in channel 1 as the calculation basis? 3. What is the 'exact' $\Delta t$ (pseudo-Wigner power function)? Two human experts decided whether a signal was an MES or belonged to one of the four artefact groups. Of a total of 97 MES, $28 \%(n=27)$ could not be detected in the distal channel. Thus, $72 \%(n=70)$ of the MES were present in botth channels and could be analysed based on the abovementioned criteria. Of these $70 \mathrm{MES}, 87 \%(n=61)$ were correctly identified off-line. We assessed altefact rejection for four different types of artefacts: changes of TCD settings, probe movement, low flow artefacts and electrocautery. The reliability of artefact rejection was $98 \%$ for setting changes $(n=382), 96 \%$ for probe movement $(n=477)$ and $98 \%$ for low flow artefacts $(n=91)$, but only $68 \%$ for electrocautery $(n=264)$. These preliminary results are promising, but need careful interpretation: $28 \%$ of the MES were not detectable in the distal SV, probably due to a poor signal-to-noise ratio (SNR) and anatomical restrictions. Electrocautery signals were insufficiently rejected. However, even an artefact rejection of $96 \%$ can be insufficient if the number of MES is very small compared to the number of artefacts. 
Introduction the automatic detection of microembolic signals (MES) with transcranial Doppler sonography (TCD) is an unresolved problem. In a comparative study of three automatic systems based on signal analysis in one sample volume (SV) and four human observers, it was shown that the automated approach was inferior in terms of the reliability of MES detection (van Zuilen et al. 1996). Recently, different research attempts have been made to enhance the specific properties of MES in the signal analysed to provide a better basis for an automated detection algorithm. These attempts focussed either on the detection of MES as the narrow band hypothesis (Roy et all. 1998) or on the discrimination between MES and artefacts as the use of two insonation frequencies simultaneously (Moehring et àl. 1997; Brucher and Russell 1997) and the application of a nonlinear algorithm (Keunen et al. 1998). Most attention, however, has been paid to the dual-gate technique (i.e., the use of two SVs placed serially behind each other). This appraach has been reported to produce excellent results in terms of sensitivity and specificity with values up to $100 \%$ (Georgiadis et al. 1996; Smith et al. 1997b; Molloy and Markus 1996\%. However, it is noteworthy that these results were obtained estimating the time difference values ( $\Delta \mathrm{t}$ ) between the MES in the two SVs manually. In contrast, two reports about the performance of a completely automated emboli-detection system based on the dual-gate approach (Georgiadis et al. 1998; Droste et al. 1997) demonstrated much lower sensitivity and specificity values. On the whole, these were comparable to those from the automated single-gated systems as examined by van Zuilen et all. (1996). Droste et al. (1997) and Georgiadis et al. (1998) gave neither specific information on how the algorithm worked nor did they report on the influence of gate separation between the SVs. It is, thus, not clear to what extent the software (signal analysis process) or the TCD settings contributed to the results that could not confirm the expected superiority of the dual-gate approach compared to the single-gate approach. We evaluated a dual-gate approach for automatic off-line MES detection based on TCD settings derived from the results of a computer simulation (Mess et al. 1999). This study had shown that the anatomy of the middle cerebral artery (MCA) plays a crucial role in the difficulties that can be encountered analysing the At values of MES passing two serially placed SVs. In contrast to the algorithm used by Droste et al. (1997) and Georgiadis et al.(1998), we used the pseudowigner distribution function (WDF) for the crucial measurements of $\Delta$ t. This signal analysis approach has been introduced for measurements on MES by Fan and Evans (1994a,1994b) and Smith et al. (1994). They showed that the WDF was superior to the fast Fourier transformation (FFT) in terms of temporal resolution. In the first instance, the data aquisition and the algorithm will be discribed, then preliminary data will be presented for the reliability of the system in terms of MES detection and artefact rejection monitoring carotid and cardilac surgical procedures. 


\section{Methods and materials}

\section{Patients, TCD settings and data acquisition}

We monitored 20 patients during carotid endarterectomy $(n=17)$ and heart surgery $(n=3)$ with unilateral TCD of the middle cerebral artery (MCA) (Pioneer, EME/Nicolet; Kleinostheim, Germany).

A $2-\mathrm{MHz}$ probe was fixed with a flexible head band in the typical temporal position. The burst length of the emitted ultrasound (US) pulses was $13 \mu S_{\text {, }}$ and the pulse-repetition frequency (PRF) was set to be sufficient for recording the highest velocities according to the Nyquist theorem. Two different 5Vs were used (Fig. 1). The proximal (i.e.x deeper) SV (SV, prox was placed in the middle of the MCA mainstem and the second $4 \mathrm{~mm}$ more distally $\left(S V_{\text {dist }}\right.$ ). In other words, the gate separation between the two SVs was $4 \mathrm{~mm}$. The "middle of MCA" was defined as the mean value of the most proximal and distal insonation depths that resulted in useful Doppler signals (median $54 \mathrm{~mm}$; range $48-58 \mathrm{~mm}$ ). The receiver gate (RG) setting was $6 \mu \mathrm{s}$ for the $5 \mathrm{~V}_{\text {prox }}$ and $2 \mu \mathrm{s}$ for the $S V$ dist. The SV length depends on both the burst length and the RG length as follows (Evans and McDicken 2000):

$S V_{\text {lengith }}=\frac{c\left(R G_{\text {lengith }}+\text { burst }_{\text {lengith }}\right)}{2}$

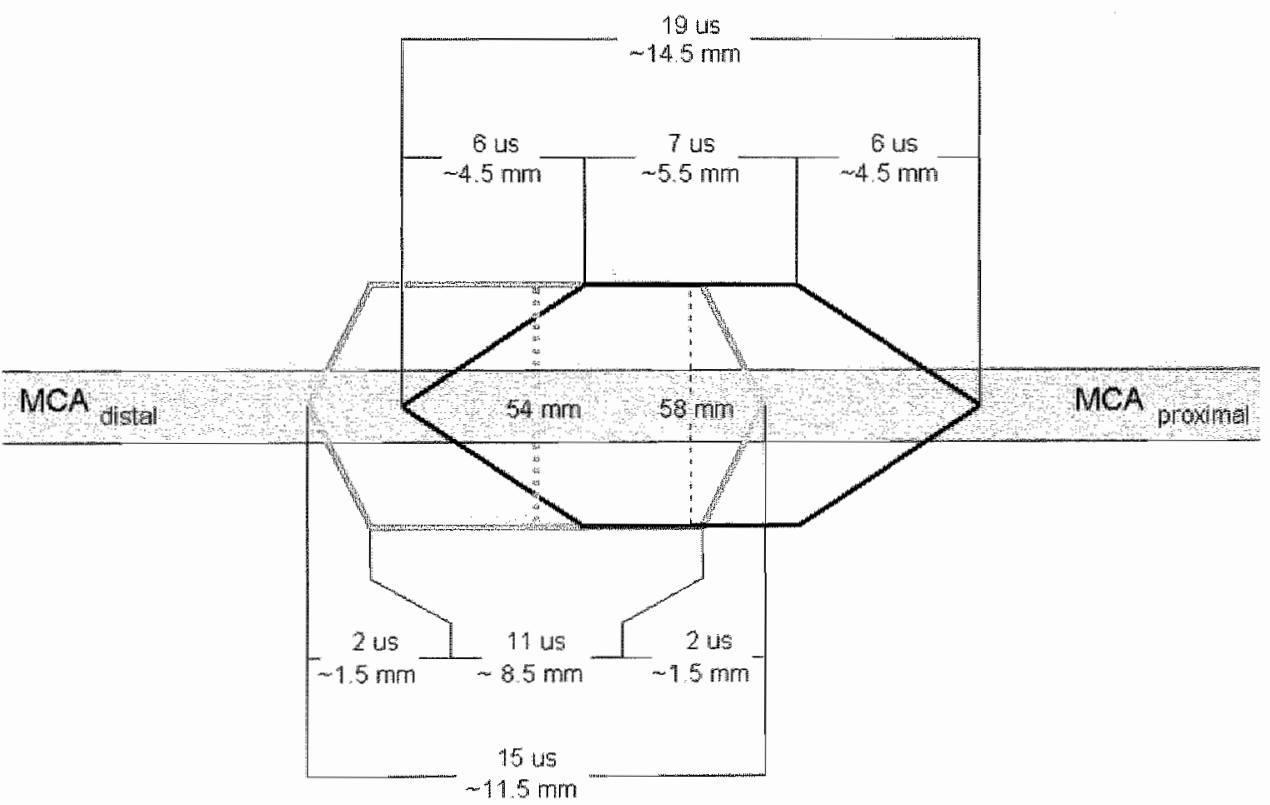

Figure 1

Figure 1

The location of the proximal SW (black) and distal $5 V$ (girey) at a depth of $58 \mathrm{~mm}$ and $54 \mathrm{~mm}$, respectiwely. The width of the $5 \mathrm{Vs}$ indicates the sensitivity, which is maximal in the center and decaying at the edgres. The lengths of the different portions of the $5 v$ s is giwen in ms and mm, whereby we assumed that the pulse ditd the gate were vectangular, and the recewer thandwidth was infinite. Because, for $5 V$ prox a receiver gate time of 6,55 was applied, the less sensitiwe part of the $5 V$ is relatively long, compared to the

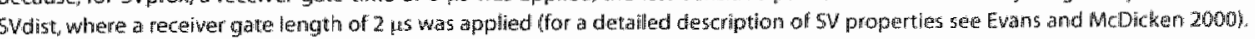


where $C$ is the velocity of US in tissue. 50 , the sum of the RG length and the burst length contribute to the maximal lengths of $S W_{\text {prox }}$ and $S V_{\text {dist }}$. These were, respectively, $14.5 \mathrm{~mm}$ and $11.5 \mathrm{~mm}$. It should be noted that short RG values cause a rapid decay of sensitivity in the axial direction of the SV; long RG values, on the contrary, cause a smoother decay. This rule applies for RG values. shorter than the burst length values (as was the case in our study).

We assumed that emboli in the MCA first pass the proximal and, subsequently, the distal SV. Moreover, normally emboli will enter and lleave both $5 V_{5}$ at, respectively, the front and rear ends of the receiver gates. If an MES has, compared to the background, a relatively low intensity, it will only be detected in the most sensitive central part of the SV, which length depends mainly on the burst length of the emitted US. However, if the MES has a relatively high backscatter it will, depending on the embolus-to-blood ratio (EBR), be detected somewhere in the less sensitive peripheral parts of the SVS (i.e., the parts that are defined largely by the RG length). In theory, using a gate separation of $4 \mathrm{~mm}$ and, respectively, a $R G$ of $6 \mathrm{~ms}$ (corresponding to $\approx 4.5 \mathrm{~mm}$ ) for $S V_{\text {prox }}$ and $2 \mu \mathrm{s}$ (corresponding to $\approx 1.5 \mathrm{~mm}$ ) for $S V_{\text {dist }}$ the largest distances between the MES in the two SVs (MES distance) will be in the range of $5.5 \mathrm{~mm}$. On the other hand, if an MES has a relatively low backscatter and is detected only in the central parts of both $5 V \mathrm{~S}_{\mathrm{s}}$ the MES distance vallue will be $4 \mathrm{~mm}$. The Doppler signals of both SVs were recorded onto an 8-channel DAT recorder (TEAC RD 110T). According to the usual practice, which is accepted as the "gold standard," wo human experts (W.H.M. and R.G.A.A.) independently analysed the recorded Doppler signals of $S V_{\text {prox }}$ for the occurrence of MES and artefacts. If both observers agreed, the signals were used for evaluation of the algorithm described below. Maximally 15 MES or artefacts were used from each patient. In total, 97 MES and 264 artefacts were used in this study. Four groups of artefacts were evaluated: change of TCD setting15, probe movement, very slow flow and electrocautery. We did not intentionally create artefacts. All signals were embedded in segments of normal Doppler signal of $10 \mathrm{~s}$.

\section{Computed data evaluation for MES}

The audio signals were digitized and then analysed applying specially developed software (B.M.T.). The algorithm used for the determination of an MES or artefact was based on the time-domain data and consisted of two consecutive steps:

\section{Analysis of intensity increase}

Doppler signals from both SVs were analysed with a 64-point FFT with $50 \%$ overlap. If there was a frequency weighted intensity increase in both channels above the background signall by 2 standard deviations (the background signal intensity was continuously calculated over a period of $50 \mathrm{~ms}$ ["running average"]), the event was regarded as a "possible MES" and referred to the second step of the algorithm. So, every significant signal intensity increase, whether being caused by an embolus or an artefact, was considered for further discrimination. 
Colculation of time difference

Calculation of MES velocity. Initially, the 64-point FFT data from channel 1 were used for further evaluation. The number of data blocks with a signal increase were counted and the central block. was calculated. To compute the velocity of the MES, the resulting 32 coefficients a belonging to the frequencies $f_{i}$ were used according to eqn (2). To enhance the contribution of small MES, we applied the cube of the coefficients instead of the square. So, the velocity was given by:

$v_{\text {erribolus }}=\frac{c f_{\text {eratolus }}}{2 f_{\text {tart }}}=\frac{c}{2 f_{\text {cart }}} \cdot \frac{\Sigma a_{i}^{3} \cdot f_{i}}{\Sigma a_{i}^{3}}$

Where $c$ is the velocity of the US in tissue, $f_{\text {embolus }}$ the frequency of the embolus, $f_{\text {carr }}$ the insonation frequency $y_{a}$ the FFT coefficient and $f_{i}$ the corresponding frequency. The calculated velocity of the embolus determined the theoretical $\triangle t$ and MES distance between the two MES in, respectively, the $S V_{\text {prox }}$ and $S V_{\text {efist }}$ Using a gate separation of $4 \mathrm{~mm}$ a value within a range of $40 \%$ to $200 \%$ (1.6-8 mm) was regarded as consistent with an MES. Additionally, the calculated velocity was used to determine the time resolution needed for an exact estimation of the velocity with the pseudo. Wigner power function.

Calculation of the starting point of an MES. The amplitude of the anallysed signal from both SVs was normalised, and the first data point in the time domain in each Doppler channel exceeding 0.2 was regarded as the starting point of the MES.

Calculation of the $\Delta t$ value and MES distance applying the pseudo-Wigner power function and final decision. The time difference between the two starting points in the two signals was calculated with a modified Wigner-Ville function (pseudo-Wigner power function), as proposed by Fan and Evans 1994a, 1994b), with the time resolution adjusted for the actual velocity of the MES. If the MES distance calculated from the $\Delta$ t value and the velocity was in the theoretically expected range (i.e., 40\%-200\%), the signal was regarded as an MES. This corresponded to an MES distance of more than $1.6 \mathrm{~mm}$. Otherwise, the signal was rejected as an artefact. 
ReSUlts in total, 97 MES were selected by the human observers. All signals were clearly visible in the time domain data of the first (i.e., proximal) channel. However, $28 \%(n=27)$ could not be seen in the second channel (f.e., there was no detectable intensity increase in the time domain signal), which excluded them from further analysis. All MES present in both channels ( $n=70$ ) were evaluated with the algorithm. Of these, $87 \%(n=61)$ were correctly classified as MES. The calculated MES distances are depicted in Fig. 2...

The Doppler signals containing artefacts (264 samples of 10 s each) resulted in alltogether 1214 detected signal increases. Of these, $1103(91 \%)$ were classified as artefacts, and $111(9 \%)$ as MES. Figure 3 shows the detected MES distance values for the 27 false-positive MES not ewoked by electrocautery. Note that all of them are within the range that was defined to be acceptable for an MES.

If we consider each artefact group separately, the reliability of artefact rejection was $98 \%$ for setting changes ( $n=382), 96 \%$ for probe movement $(n=477), 98 \%$ for low flow artefacts $(n=91)$ and $68 \%$ for electrocautery $(n=264)$. All results are summarized in Table 1 .

Table 1

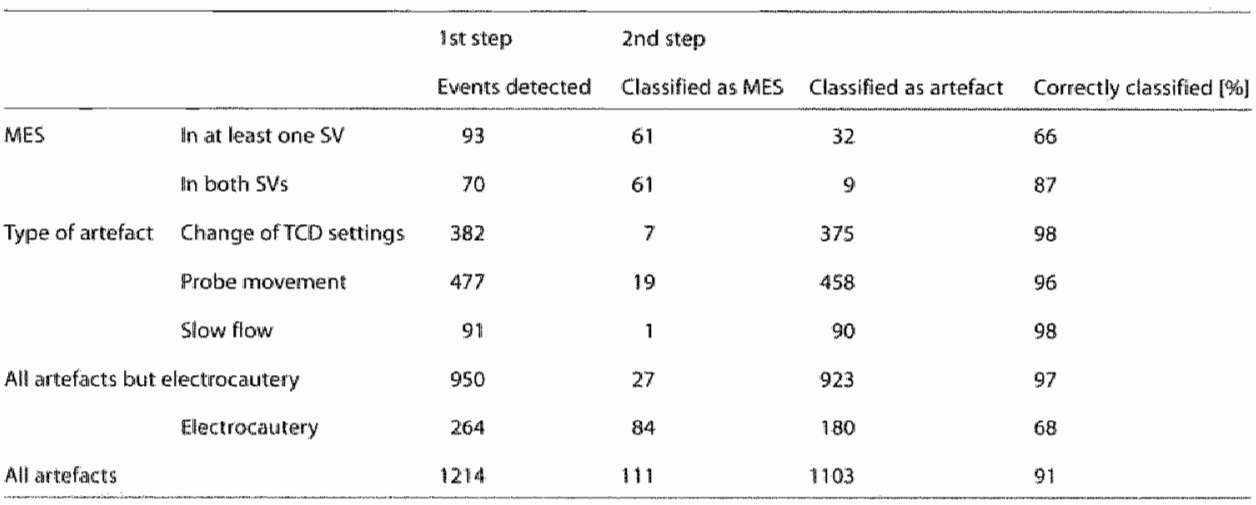

Five different signal types. Ihtes and 4 artefact categoriesh were evaluated. The first column shows how often the frst step of the algorthm (intensity increase in both channels, measured with a 64 point FFT) reacted on the signals presented. The second and third column display the results or the second step of the algorithm (MES distance measurement and decisian, whether a signall was an MES or an artefact). 


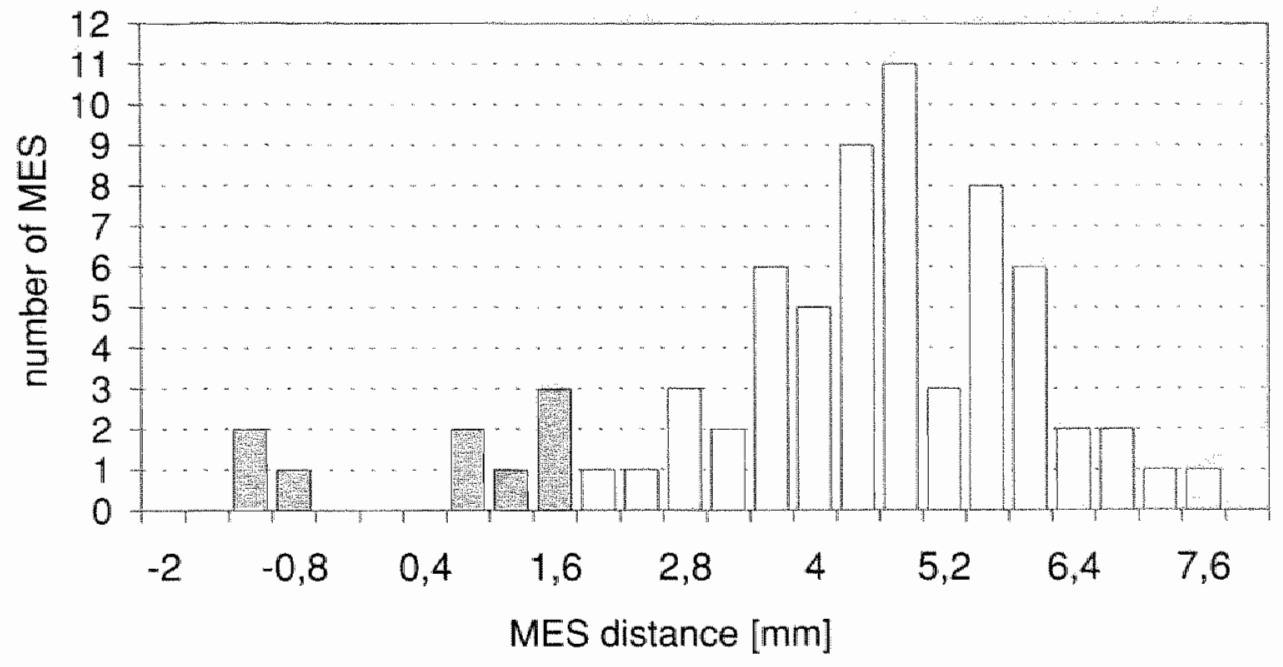

Figure 2

\section{Figure 2}

The $x$-axis shows the calculated distance in mm between the MES that occur in both $5 V \mathrm{~s}$ ( $n=70$. The $y$-axis shows the albsolute number of MES with a giwen walue. To separate the MES from antefacts, a cut-off value of $1.6 \mathrm{~mm}$ was chosen (MES $>1.6 \mathrm{~mm}$ ); falsenegative MES are indicated with grey bars.

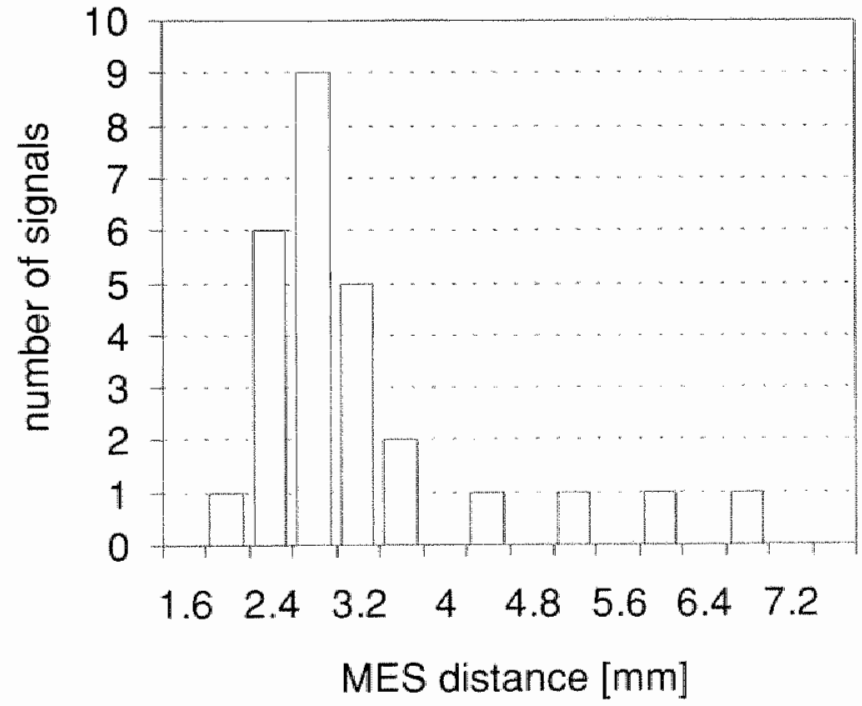

Figure 3

Figure 3

The MES distance walues of the 27 artefacts not evoked by electrocattery that were accepted as Mts are displayed on the sranis, with the quantily of AVES on the y-axis. 
DiSCUSSION we proposed a new automatic MES-detection algorithm based on the dual-gate technique. The preliminary results of the analysis of 97 MES and 1214 artefacts are promising, but need careful interpretation.

\section{Nondetected MES}

With respect to the application of the dual-gate technique an appropriate setting of the SVS in terms of gate separation is crucial Actually, two conflicting demands have to be met: 1 . To create reliable At values, the SVs have to be placed with a distinct gate separation, and 2. To avoid a significant variation of MES characteristics, the SVs have to be placed as close as possible to each other.

In this study, we showed that in our TCD settings with a relatively short SV distally lacated in a larger proximal SV, the MES distance values measured were in good agreement with the theoretically calculated walues. Concerning the second demand, like others (5mith et al. 1997a; Georgiadis et al. 1996), we also observed, in spite of a significant overlap of the two SVs, so-called "missing emboli." Because only $75 \%$ of all MES occurred in both SVs, this was actually the main obstacle to a more successful automatic detection with the dual-gate technique.

Possibly, this high number of missing emboli can be explained by the relatively poor SNR in the $S V_{\text {edis }}$. After all, the short receiver gate time of this $S V$ results in a significantly lower backscatter for further analysis. Additionally, our method of signal storage on DAT also deteriorates the SNR. During the process of recording and analysis, the Doppler signals are transformed 4 times from digitall to analog format or vice versa.

Because the $S \mathrm{~V}_{\text {dist }}$ lies for the greater part in the distal half of the $S \mathrm{~V}_{\text {prox }}$ it is unlikely that one quarter of the MES vanish into perforating arteries that originate from the MCA mainstem. On the other hand, in many patients, the MCA mainstem is quite curved and this results in significantly different insonation angles for the two SVs (Mess et al. 1999). In this respect, it is important to underline that the majority ( $85 \%)$ of "missing emboli" were noticed in 3 of the 20 patients inwestigated.

Of all 70 MES that occurred in botll $S V S, 9(13 \%)$ were not identified by the algorithm. If the threshold for MES acceptance in the second step of our algorithm is decreased from $1.6 \mathrm{~mm}$ to $0.8 \mathrm{~mm}$ MES distance (see Fig. 2), the sensitivity increases to $96 \%$.

\section{Artefact rejection}

For artefact detection, we analysed 264 samples of Doppler signals. This resulted in 1214 signal increases detected during the first step of the algorithm. This high number reflects the lack of clearcut definitions of duration of an artefact. Especially probe movement and electrocautery were events that could last up to several s. In a former study, we showed that, in our institution, artefacts caused by electrocautery create significant $\Delta t$ values and, therefore, were insufficiently (68\%) detected by the dual-gate technique (Mess et al. 1997). However, this phenomenon probably 
depends on the electrocautery device used and electrocautery can easily be detected by an antenna that picks up the electrical field.

\section{Comparison with ather automated systems}

The present study shows that the main disadvantage of the dual-gate technique is the problem of "missing emboli." Two other studies on automatic emboli detection have been performed with a commercially available dual-gate system (Droste et al. 1997; Georgiadis et al. 1998). Regarding emboli detection, both studies reported high values for sensitivity and specificity during heart surgery and in patients with mechanicall heart valves. Howewer, the authors did not describe the crucial aspects, of the algorithm used. lin these studies, it remained unclear how the decisition between MES and artefacts was made, and it was not stated whether the raw data (time domain) or the FFT-processed signal (frequency domain) were used for the $\Delta t$ calculations. Finally, the authors did not report on "missing embolit."

Conclusions in conclusion, it could be shown that the dual-gate techinique, indeed, bears considerable potenthal as a reliable provider of MES data that can be further automatically analysed. Concerming our preliminary data, the majority of MES that appeared in both $5 \mathrm{Vs}(87 \%)$ were correctly identified by the newly developed algorithm. However, $28 \%$ of the MES detected by the human experts were missed because they were not contained in the $S \mathrm{~V}_{\mathrm{d} \mid \mathrm{st}}$. With the exception of electrocautery, artefact rejection was excellent. Compared to other existing automatic MES-detection systems, our data are encouraging enough to warrant further studies with more MES of different natures. The use of different threshold parameters for MES intensity or MES distance values derived from a receiver-operated curve analysis could result in optimal detection settings for different examination situations.

\section{Acknowledgements}

The authors thank Prof.D.H. Evans for helpful comments during the preparation of this manuscript. 


\section{References}

Brucher R, Russell D. Differentiation between gaseous and solid microemboli using multi-frequency Doppler (abstract). Eur J Uitrasound 1997; 5 (suppl 1):540.

Droste DW, Hagedorn G, Nötzold A, Siemens HJ, Sievers HH, Kaps M. Bigated transcranial Doppler for the detection of clinically silent circulating emboli in normal persons and patients with prosthetic heart valwes. Stroke 1997; 28:588-592.

Evans DH, MCDicken WN: Doppler ultrasound; physics, instrumentation and signal processing, 2nd ed. Chicester, Wiley $\&$ Sons 2000.

Fan L، Evans DH. Extracting instantaneous mean frequency information from Doppler signals using the Wigner distribution function. Ultrasound Med Bifol 1994a; 20:429-443.

Fan L, Evans DH.A real-time and fine resolution analyser used to estimate the instantaneous energy distribution of Doppler signals. Ultrasound Med Biol 1994b; 20:445-454.

Georgiadis D, Goeke J, Hill M, König M, Nabovi DG, Stögbauer F, Zunker P, Ringelstein EB. A novel technique for identification and Doppler microembolic signals based on the coincidence method; in vitro and in vivo evaluation. Stroke $1996 ; 27: 683-686$.

Georgiadis D, Wenzel A, Zerkowski HR, Zierz S, Lindner A. Automated intraoperative detection of Doppler microembolic signals using the bigate approach. Stroke 1998; 29:1377-139.

Keunen RWM, Stam CJ, Tavy DL, Mess WH, Titulaer BM, Ackerstaff RGA. Preliminary report of detecting microembolic signals in transcranial Doppler time series with nonlinear forecasting. Stroke 1998; 29:1638-1643.

Mess WH, Titulaer BM, Ackerstaff RGA. Discrimination and characterization of emboli: old and new aspects. In: Klingelhöfer J, Bartels E, Ringelstein EB, ed. New Trends in Cerebral Hemodynamics and Neurosonology. Amsterdam: Elsevier, 1997:355-363.

Mess $W_{1}$, Titulaer BM, Ackerstaff RGA. Middle cerebral artery anatony and characteristics of embolic signals - a dual gate computer simulation study. Ultrasound Med Biol 1999; 25:in press.

Moehring MA, Spencer MP, Radford RL, MCDaniel MD, Mattson GA. Low frequency annular transducers for detecting and characterizing microemboll in cardiopulmonary bypass (CPB) flow circuits with the embolus to blood power ratio (EBR) (abstract). Eur J Ultrasound 1997;5 (suppl 1):541. 


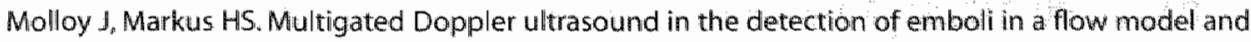
embolic signals in patients. Stroke 1996; 27:1548-1552.

Roy E, Abraham P, Montrésor S, Baudry M, Saumet $J L$. The narrow band hypothesis: an interesting approach for high-intensity transient signals (HITS) detection. Ultrasound Med Bilol 1998; 24:375-382.

Smith JU, Evans DH, Fan L, Thrush AJ, Naylor AR. Procesising Doppler ultrasound signals from bloodborne emboli. Ultrasound Med Biol 1994; 20:455-462.

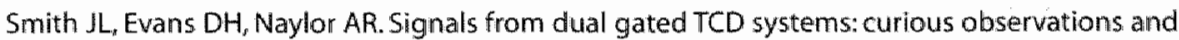
possible explanations. Ultrasound Med Biol 1997a; 23:15-24.

Smith JL, Evans DH, Fan L, Bell PRF, Naylor AR. Differentiation between emboli and artefacts using dual-gated transcranial Doppler ultrasound. Ultrasound Med Biol 1997b; 22:1031-1036.

van Zuilen EV, Mess WH, Jansen $C_{f}$ van der Tweel I, vam Gijn J, Ackerstaff RGA. Automatic embolus detection compared with human experts; a Doppler ultrasound study. Stroke 1996; 27:1840-1843. 
5

Microembolic Signal description: A reappraisal

based on a customized digital post-processing system

Ultrasound Med Biol 2002;28:1447-1455 

Abstract The high variability in presence and signature of microembolic signals (MES), detected with transcranial Doppler sonography (TCD) in the middle cerebral artery (MCA), cannot be explained with the currently available published data. We applied customized postprocessing on the radiofrequency (RF) signal of a standard TCD system. The spatial resolution was on the order of $2 \mathrm{~mm}$, depending only on the length of the ultrasound (US) burst emitted. The amplitude of clutterfiltered RF signals was color-coded and plotted as a function of time and depth (range $30 \mathrm{~mm}$ ). Additionally, 128 point fast Fourier transforms (FFTs) (50\% temporal overlap) were calculated, visualizing both the background Doppler spectrum and the MES. We evaluated 122 gaseous MES from two patients during cardiac surgery and 52 particulate MES from four patients after carotid endarterectomy. Both MES categories, showed comparable properties: they appeared in the RF amplitude plot as rather straight lines of increased intensity, indicating that the velocity remained approximately the same while they passed the US beam. The velocity calculated from the amplitude plat never exceeded that of the background Doppler spectrum. Various "MES patterns" could be identified with respect to the depth range at which the MES were visible. A quarter of the gaseous MES changed their direction at a specific depth, suggesting that the MES entered a branch (e.g.n an $\mathrm{M} 2$ artery or the anterior cerebral artery). In the FFT analysis, these MES contained both positive and negative frequencies. It is concluded that MES show consistent signature patterns in the amplitude-time plots and that the previously reported variability of MES appearance in conventionall Doppler systems is an artefact caused by relatively large signal amplitudes and sample volumes. 
Introduclion Transeranial Doppler sonography (TCD) can be used to detect microembolic signals (MES) in the middle cerebral artery (MCA). Their presence is characterized by a sudden increase of the backscattered Doppler signal amplitude and causes a typical chirping sound, mostly accompanied by a comma-like or vertical bar in the fast Fourier transform (FFT) spectrum. There is a high variability in the presence and signature of MES, even in the same patient with unchanged TCD settings, that cannot be explained with the currently avallable published data (Smith et al. $1997 \mathrm{a}, 1997 \mathrm{~b})$. The inconsistent appearance of MES in FFT spectra might be the major culprit of the rather disappointing results of the dual gate technique, which relies on the conception that an MES serially passes two sample vollumes (SV), positioned sequentially over the MCA. Ideally, a signal increase in the two SVS within a distinct time interval would undoubtedly separate MES from artefacts. Computer simulations confirmed that the different appearance of the MES in the two SVS largely depended on the anatomy of the MCA (Mess et al. 1999!. In an automatic MES-detection algorithm based on the dual gate technique, it will cause false-negative results (Droste et al. 1997; Georgiadis et al. 1998; Mess et al. 2000).

In addition to the MCA anatomy, size and position of the applied SVs are presumably responsible for the inconsistent data published on MES signature. The design of conventional Doppler systems is characterized by two major drawbacks that will render an exact description of an MES impossible. First, the rather long ultrasound (US) bursts emitted, which can result in SV sith a length of up to several $\mathrm{cm}$ in the case of gaseous MES (5mith et all. 1998), are responsible for an inferior spatial resolution. Second, most commercially available TCD systems show the spectrum of the Doppler signals originating from one or a limited number of positions in depth. As a result of the FFT-based transfer from the time domain to the frequency domain also, the temporal resolution is limited. For MES detection and visualization, the transfer from time to frequency domain is not necessary and, sometimes, even unwanted. The Wigner analysis (Fan and Evans 1994), the application of wavelets (Aydin et al. 1999), and the use of frequency filtering (Markus and Reid 1999) have been proposed for a more accurate description and selective detection of MES. However, these approaches still relied on the diemodulated Doppler signal and, consequently, rather large SVS.

We propose a system that acquires the unprocessed radiofrequency (RF) US signals over a large depth range, allowing off-line processing with a high dynamic range and the best spatial resolution. This system offers the possibility of following the course of the MES as a function of time and depth in the basal cerebral arteries. The results for both particulate and gaseous MES are presented. 


\section{Materials and methods}

\section{Description of the data-acquisition system}

From a standard TCD system (Multidop $X 4$, DWL, Sipplingen, Germany) the RF signal, the emission trigger $(\mathbb{E T})$ and the synchronous sample clock $(\mathrm{CLK} ; 16 \mathrm{MHz})$ were made externally available.

These signals were connected to a custom-made personal computer (PC) data-acquisition interface that digitized the RF signals at $16 \mathrm{MHz}$ with a dynamic range of $72 \mathrm{~dB}(12$ bits; Fig. 1$)$. The temporal resolution of the system was defined by the pulse repetition frequency (PRF; $5.99 \mathrm{kHz}$ ). Although the RF signals were acquired with a spatial sample distance of $48 \mathrm{um}(16 \mathrm{MHz})$, the actual spatial resolution was mainly dependent on the length of the emitted US burst ( $\sim 3$ to 4 us), which corresponded to an axial resolution of 2 to $3 \mathrm{~mm}$. Based on the bandwidth of the received signal, the calculated spatial resolution was only slightly worse and, also, on the order of 2 to $3 \mathrm{~mm}$. The data were stored into the PC memory buffer (RAM), and eventually transferred to hard disk in case an MES was audibly present. A single recording of RF signals covered maximally a depth range of $30 \mathrm{~mm}$ lasting 10 s. To restrict the sampled data to the time interval containing the $M E S_{\text {, }}$ a sampling time of 2 to 35 was sufficient. The system applied here is a modification of a RFacquiring system previously described for the assessment of vessel wall and hemodynamic properties of large arteries (Brands et al. 1999).

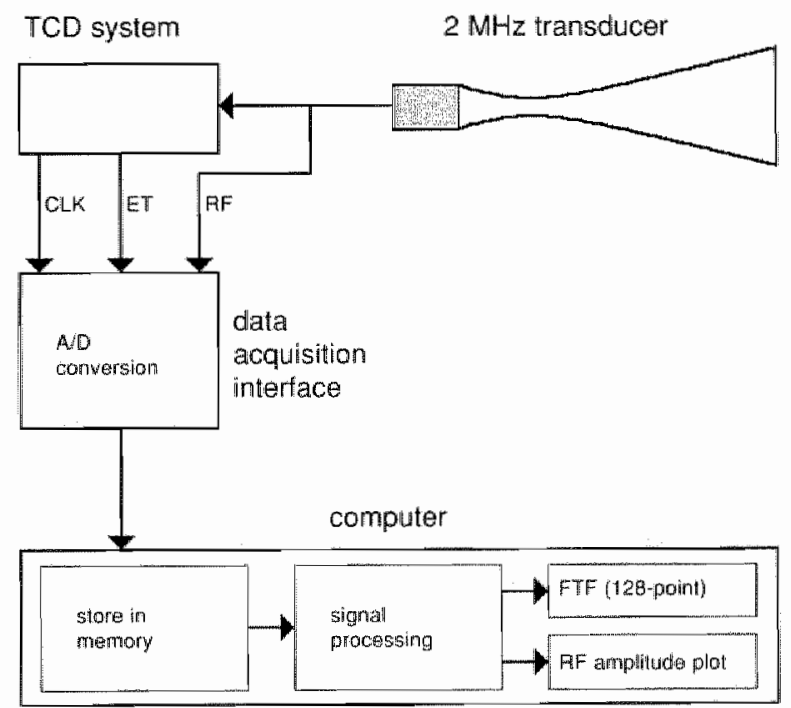

\section{Figure 1}

Block diagram of the RF signal-acquisition and processing system. The RF signal was acquired from the TCD system before any

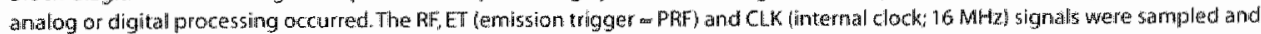
then stored into RAM memory of an extemal computer. In case a 5witch was pressed the signals were stored onto has disk and further processed. Finally, an FFT spectram of the whole signal sampled and an RF amplitude plot over a selected thme range were calculated. 


\section{MES analysis}

A 2.MHz probe was fixed over the right temporal bone window. The burst length of the emitted US pulses was set to 3 to $4 \mu \mathrm{s}$, and the depth range containing a Doppler signal from the MCA was estimated, based on the audio output of the Doppler device (usually 20 to $30 \mathrm{~mm}$ ). This depth range, then, was used for the depth adjustment of the data-acquisition system. MES were collected by pressing a switch at the moment that an MES was acoustically noticed. The RF signals within $1 \mathrm{~s}$ before and after the MES appeared were captured and stored on hard disk.

After the monitoring session had been completed, 128 point FFTs ( $21 \mathrm{~ms} ; 50 \%$ temporal overlap) were calculated, visualizing both the background Doppler spectrum and the MES. The FFT plot covered the complete sample, being 2 to 3 s long and, thence, allowed us to choose the appropriate time window for the amplitude plots, which covered about 50 to $100 \mathrm{~ms}$. Each point of the sampled RF signal over time contains, the Doppler signal for a given depth. To remove the clutter, the Doppler signal was passed through a second order Butterworth high-pass filter. The cut-off frequency of the recursive filter was set at $50 \mathrm{~Hz}$, which is the lowest possiblle frequency according to the selected time window for FFT analysis. The instantaneous envelope of the amplitude of the clutter-filtered RF signals was color-coded and plotted as a function of time (saimple interval $0.17 \mathrm{~ms}$ ) and depth (sample interwal $0.05 \mathrm{~mm}$ ). This amplitude plot was used for the description of MES characteristics. Figure 2 gives an example of an MES first as amplitude plot, second as a function of depth at a

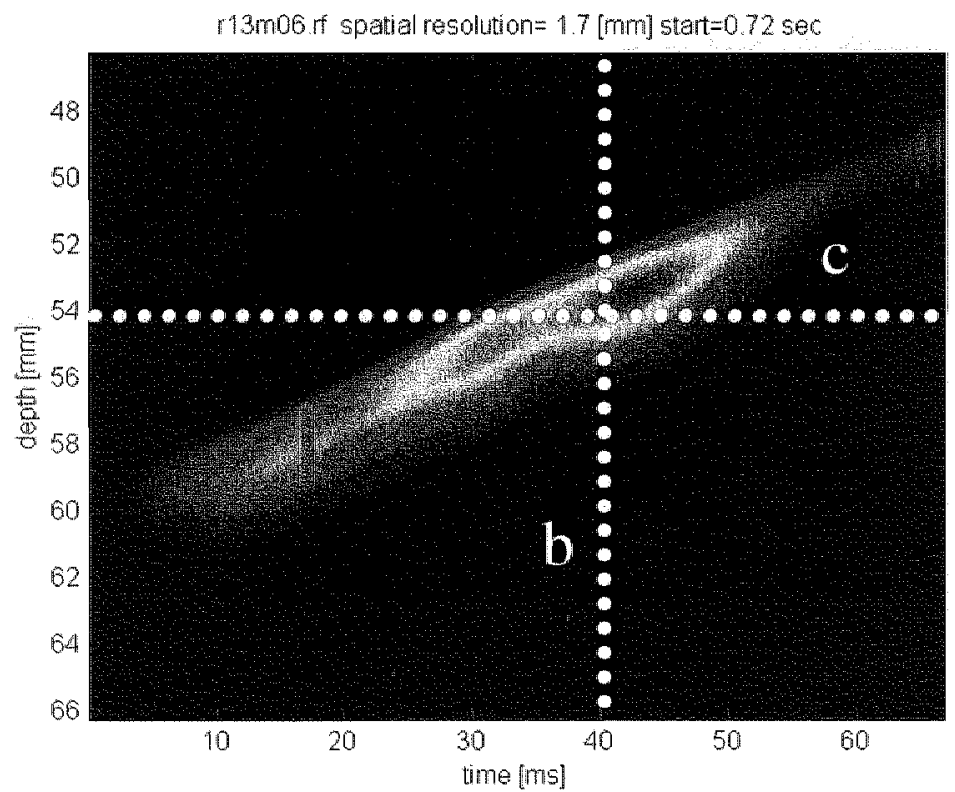

Figure $2 a_{i}, b$ and $c$

MES in the amplitude plot as a function of tirne and depth. In (a) the amplitude plot, the MES is visualized as a color-coded intensity increase. The green, yellow and red tones, respectively, indicate higher walues for the intensity of the RF signall. The MES appears in the amplitucte plot at a depth (b-axis) of approximately $60 \mathrm{~mm}$ and exhibits a thicknes. of approximately 2 mm (red bar), which is in accordance with the spatial resolution. In the course of time ifrom $\sim 10$ to 60 mus; $x$-axis) the appesance shifts from about 60 to $52 \mathrm{~mm}_{\mathrm{t}}$ according to a movement of the MES towart the probe. The MES in the RF signal cam also be wisualized as a function of (b) depth (e.g. at $t=40 \mathrm{~ms}$ ) on (c) time (e.g. at $54 \mathrm{~mm}$. corresponding to the white dashed lines in (a). 


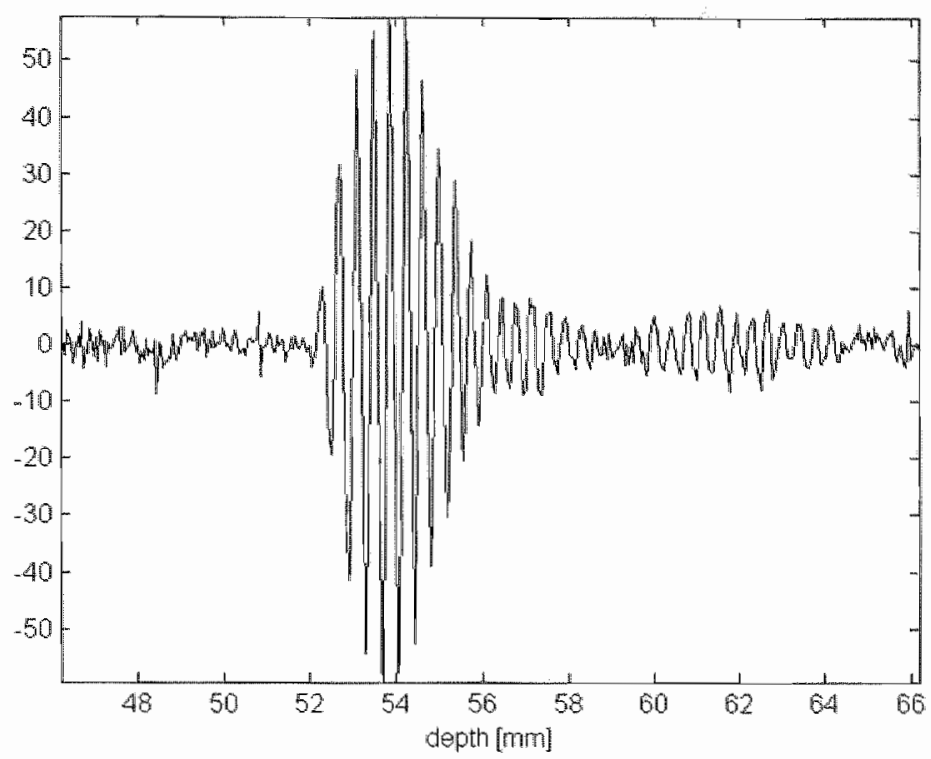

Figure $2 \mathrm{~b}$

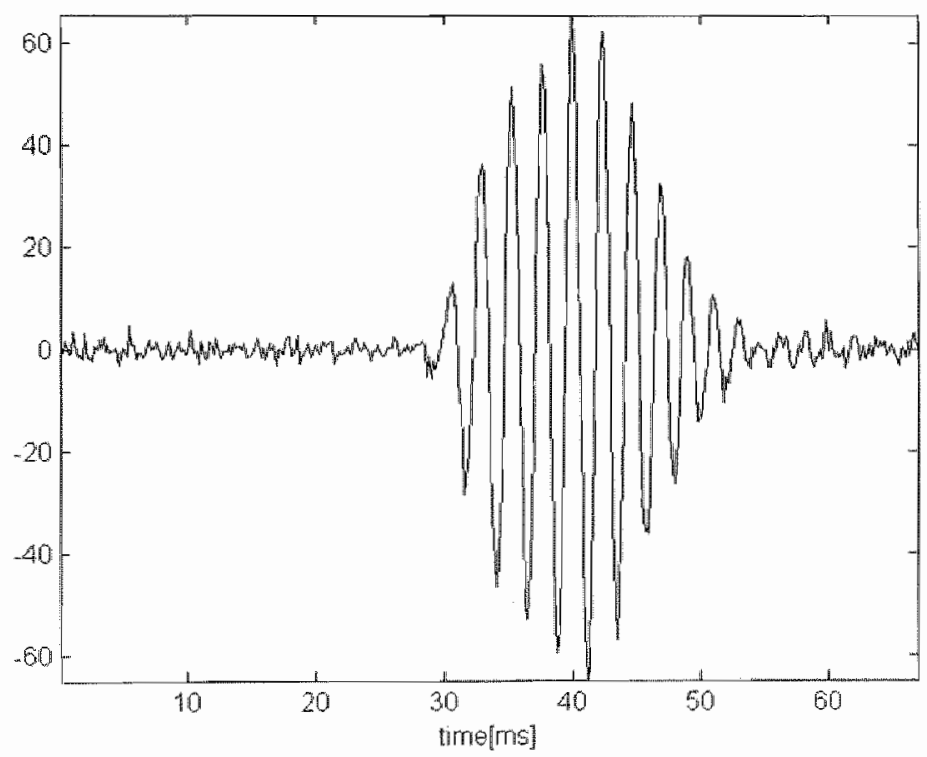


Figure $3 a$

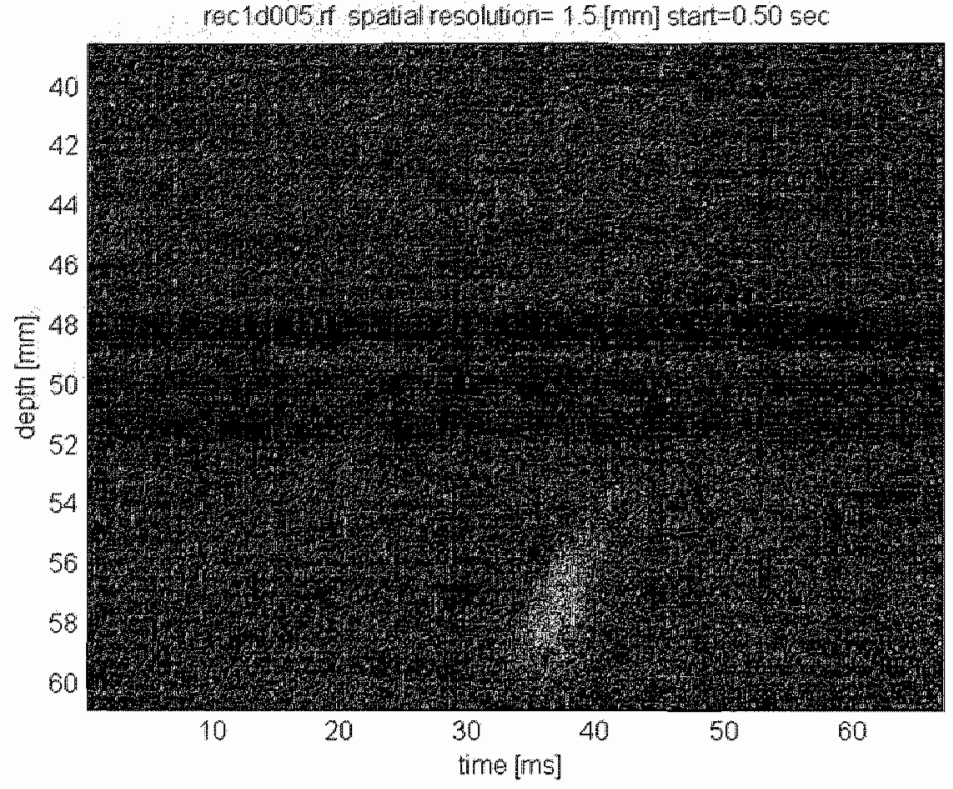

b

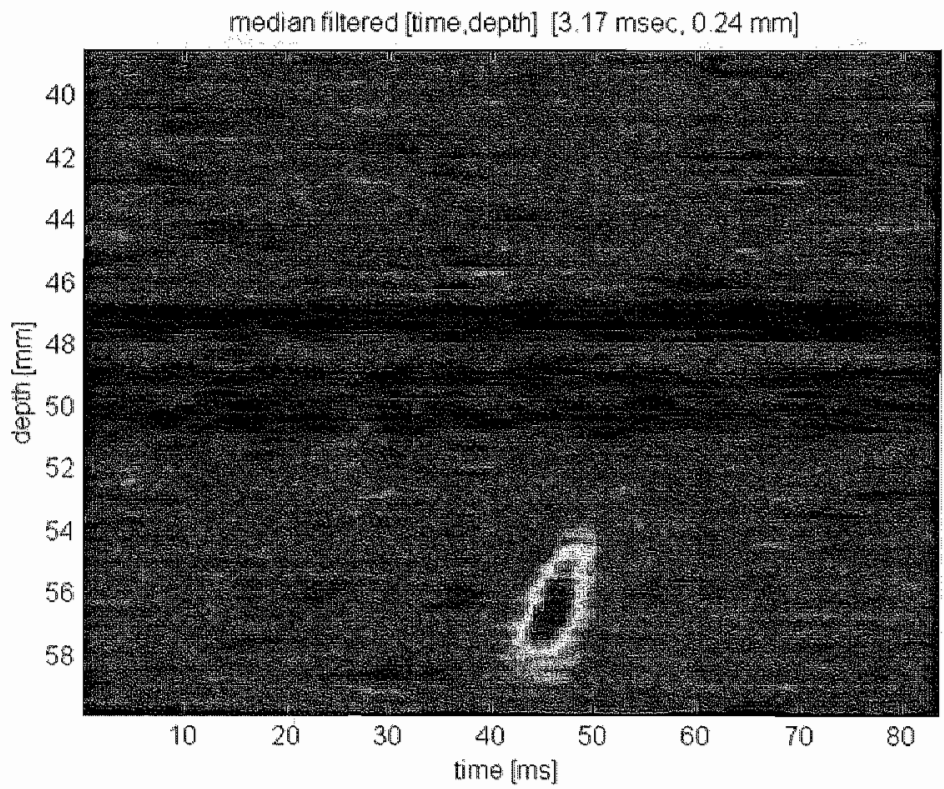

Flgure $3 a$ and $b$

Median filter for MES with a low SNR. The same MES is displayed in an amplitude plot (a) without and (b) with 5 * 5 ngedian filtering Note that the application of the filter "smoothes" the appearance of the MES. Despite the improved visualization that allowws fior the wetermination of the depth tange of the MES, it is still not possible to reliably estimate the direction and velocity of this MES. 


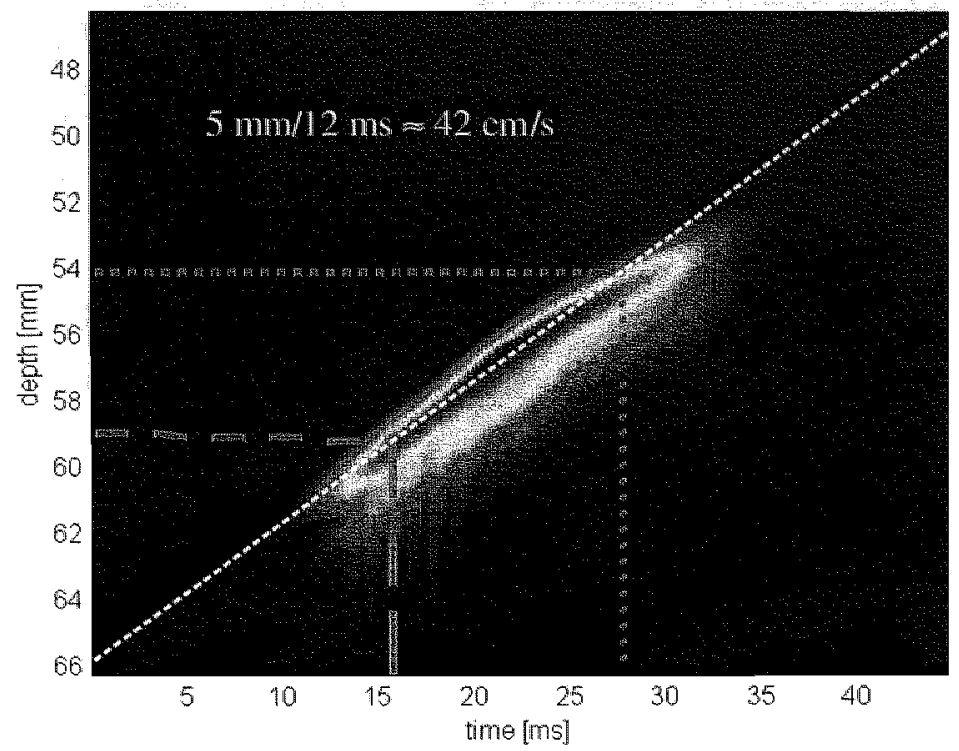

Figure 4a

rst301.bin GateStart $=49.3 \mathrm{~mm}$, GateLength $=9.2 \mathrm{~mm}$, 1 ew $=28.4 \mathrm{~ms}$

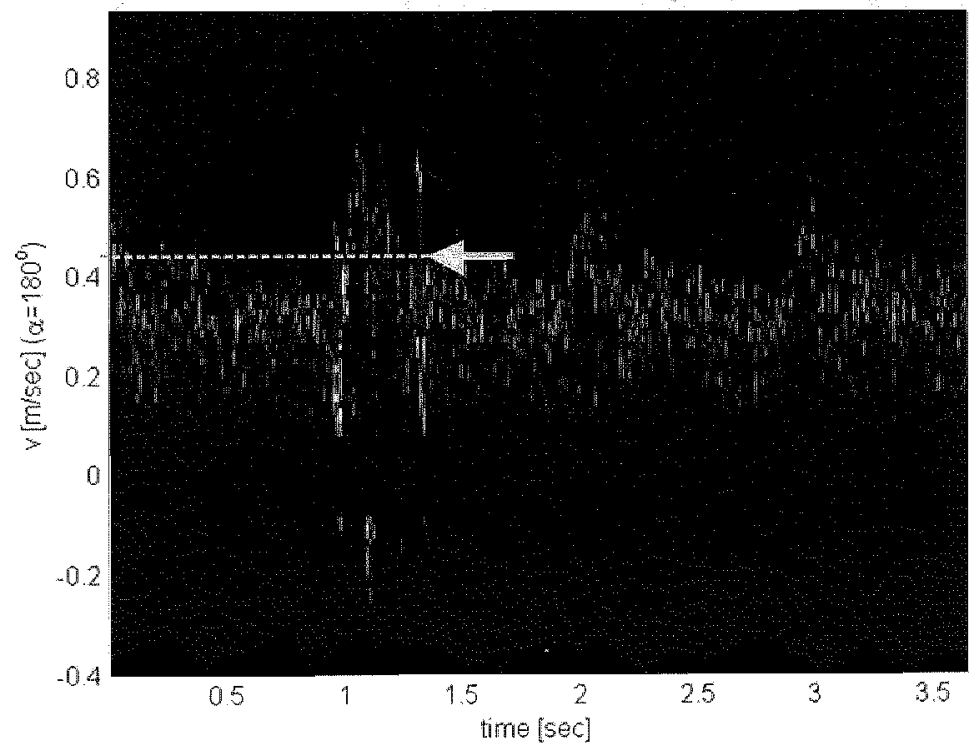

Figutire $4 a$ and $b$

MES welocity calculation. (a) The velocity of an MES was dletermined by drawing a tangential line through the area of incueased intensity and calculating the steepness of that line, which reflects the velocity ( $\Delta d / \Delta t)$, (b) The fFT whows the same MES (arrow) as a vertical bas that exceeds the Doppler backgroundi (-) the velocity as calculated firom the amplitude plot obviously, a meaning full velocity calculation is not feasible. (c) If, according to Fig. $2 \mathrm{~b}$, the amplitude increase is plotted for twwo different depths 15 mum distance; indicated in (al with blue idashed lines), a time difference can be calculated that vields, in this example, aweloctly that is comparable to that calculated on the basis of the amplitude plot (i.e., $46 \mathrm{~cm} / \mathrm{s}$ ). 

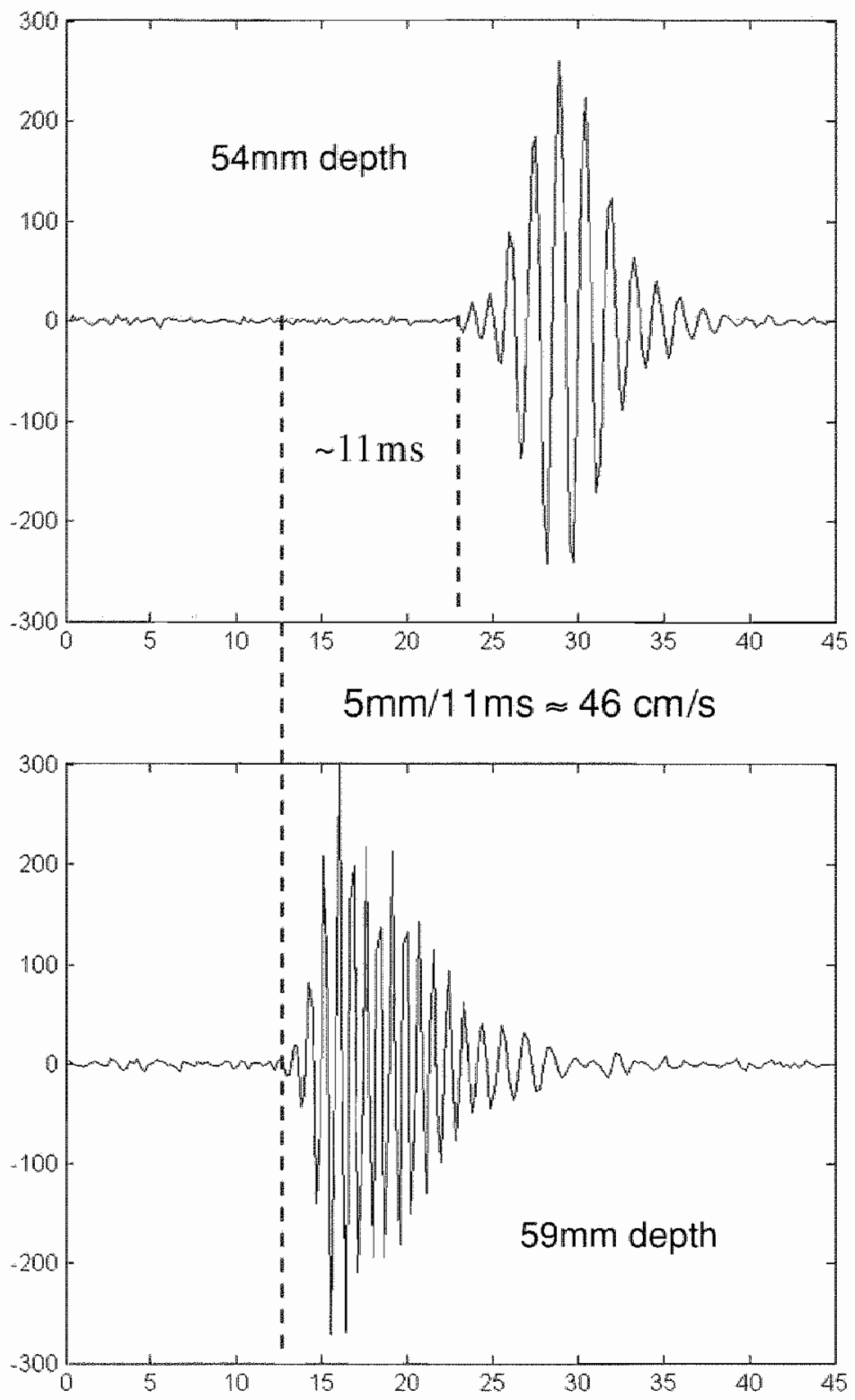
given time point and, third, as a function of time for a given depth range. For the analysis of particulate MES, which typically show less intensity increase than gaseous MES compared with the Doppler background signal, a 2-D median filter was applied for the amplitude plot (median of $5 * 5$ sample points in depth and time with $80 \%$ overlap), resulting in a better signal-to-noise ratio (SNF) at the expense of a negligible loss of temporal and spatial resolution (see Fig. 3). Figure 4 illustrates that the velocity of MES can be obtained by drawing a tangential line through the area of increased intensity.

We evaluated 122 gaseous MES from two patients at the final stage during cardiac surgery (cluring weaning off pump; this stage is characterized by a large number of air bubbles stemming from air that has been entrapped in the heart) and 52 particulate MES from four patients after carotid endarterectomy (MES sampled at least more than $30 \mathrm{~min}$ after wound closure). The MES were sampled during routine TCD monitoring procedures. The MES were described in terms of depth range, motion direction and velocity. 
ReSUlls An overview of all MES is given in Table 1 ; the results for the analysis of gaseous and particulate MES are described separately.

\section{Talble 1}

Overvien of all MES anatysed.

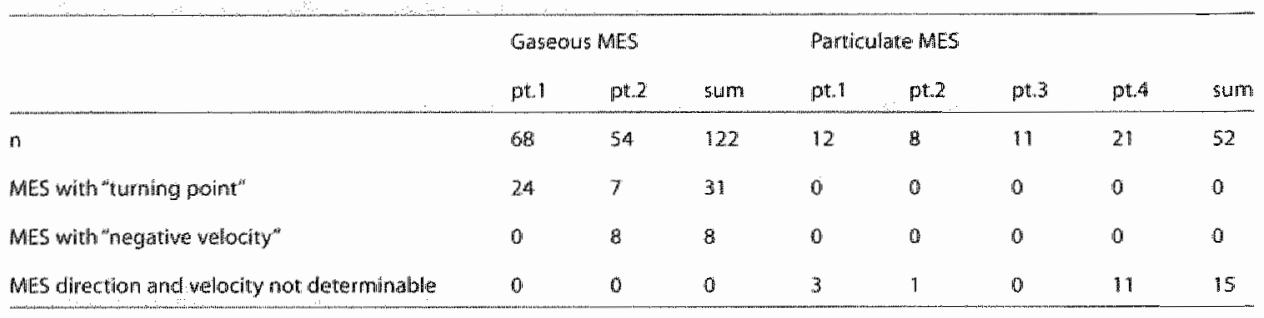

Two categones of MES were awaluated. First, total of 122 gaseous MES from two paltients were recorded in the final stage of cardiac. valve replacement surgery, second, a total of 5.2 particulate MES from four patients were recorded after canotid endarterectomy. For all patients, the number of MES with at changing welocity, those with a movement onlly away from the probe and those in which the direction and velacity could not be determined, is given.

\section{Gaseous MES}

All signals recorded contained MES with a sufficient SNR to allow good visibility and interpretation. The MES in both patients showed comparable properties. They appeared in the RF amplitudie plot as straight lines of increased intensity, indicating that the velocity did not change as they passed the US beam (Fig. 2a). The velocity calculated from the amplitude plot never exceeded the background Doppler velocity (Fig. 4). Various "MES patterns" could be identified with respect to the depth range at which the MES were wisible (Fig. 5). Figure 6 shows the consistent narrow depth rangle, about $20 \mathrm{~mm}$ (Fig 6al; subject 1) and $10 \mathrm{~mm}$ (Fig. 6b; subject 2), within which MES were dletected; the mean length was $4.5 \mathrm{~mm} \pm 1.6(\mathrm{SD})$.

In the majority of cases, the intensity increase appeared at greater depths and moved toward the probe. However, 31 of 122 MES changed their direction at a specific depth (see Fig. 7), compatible with an initial movement toward and consecutively away from the probe. The velocity and depth range of the two segments of the time course were not the same. The depth of the turning point showed a remarkable consistency, with a variation of only $2 \mathrm{~mm}$, which is within the measurement accuracy of the system (Fig. 8). The exact point of changing direction was not visible due to the application of the clutter filter $(50 \mathrm{~Hz})$. FFT analysis showed that these MES induced both positive and negative frequencies.

In the second patient, 8 of 54 MES showed a movement solely away from the probe, which always occurred at the largest depths (Fig. 6b). 


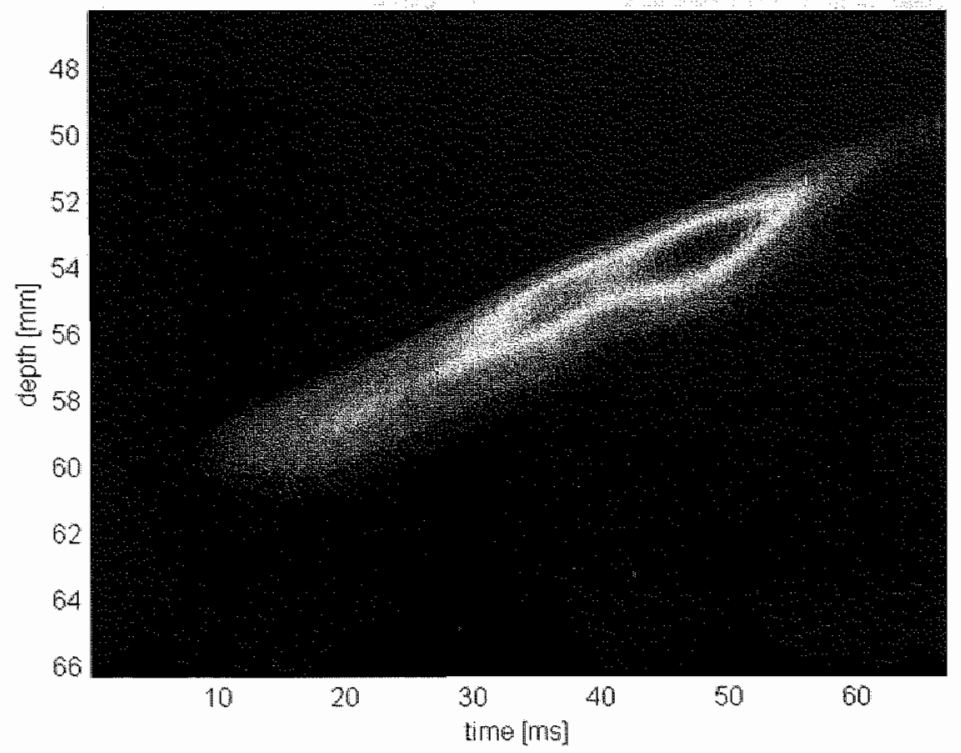

Figure 5a

r13mo6 if spatial resolution= $=1.7$ [mm] start=1.15 sac

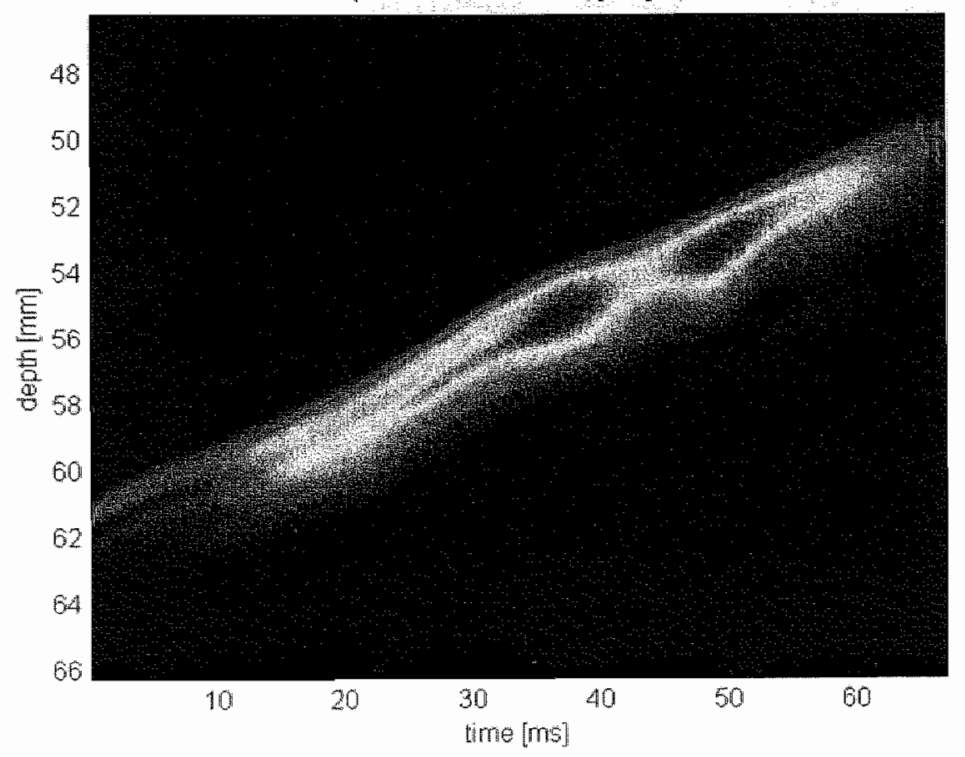

Two MES of the same patient appearing at the same depth range $(-501060 \mathrm{~mm}$. 


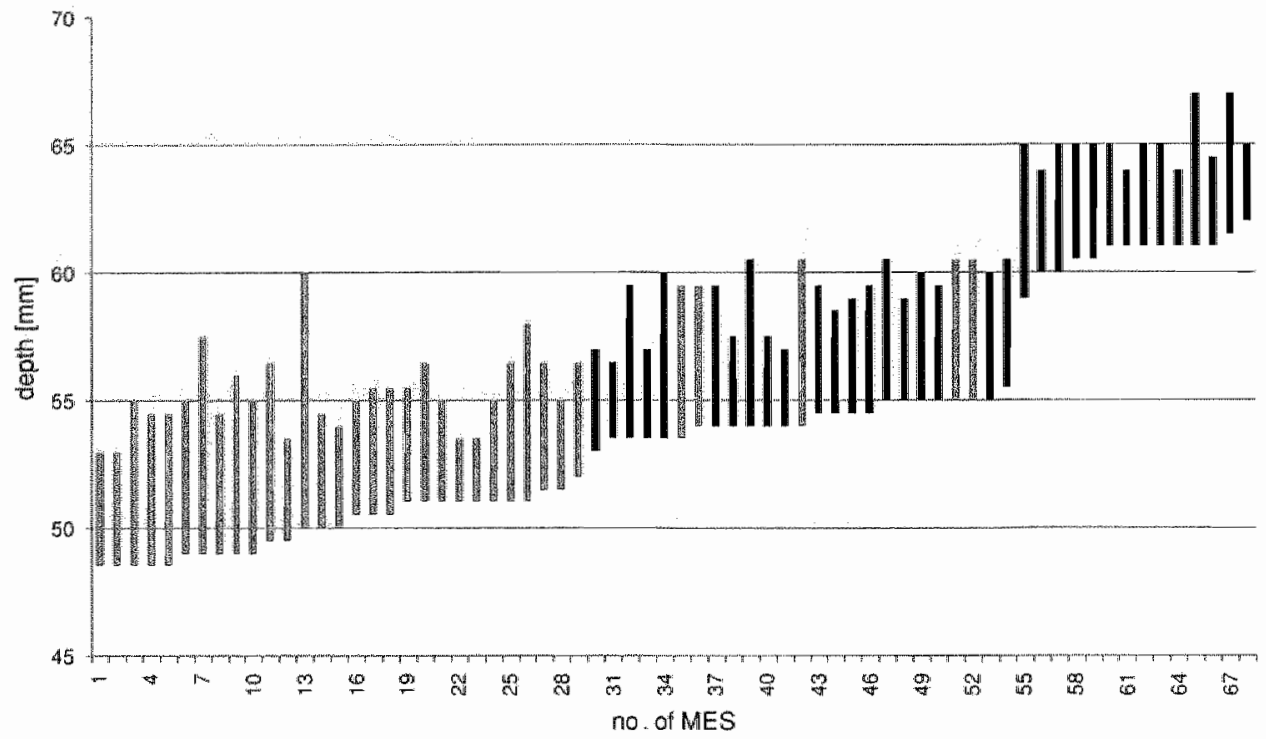

b

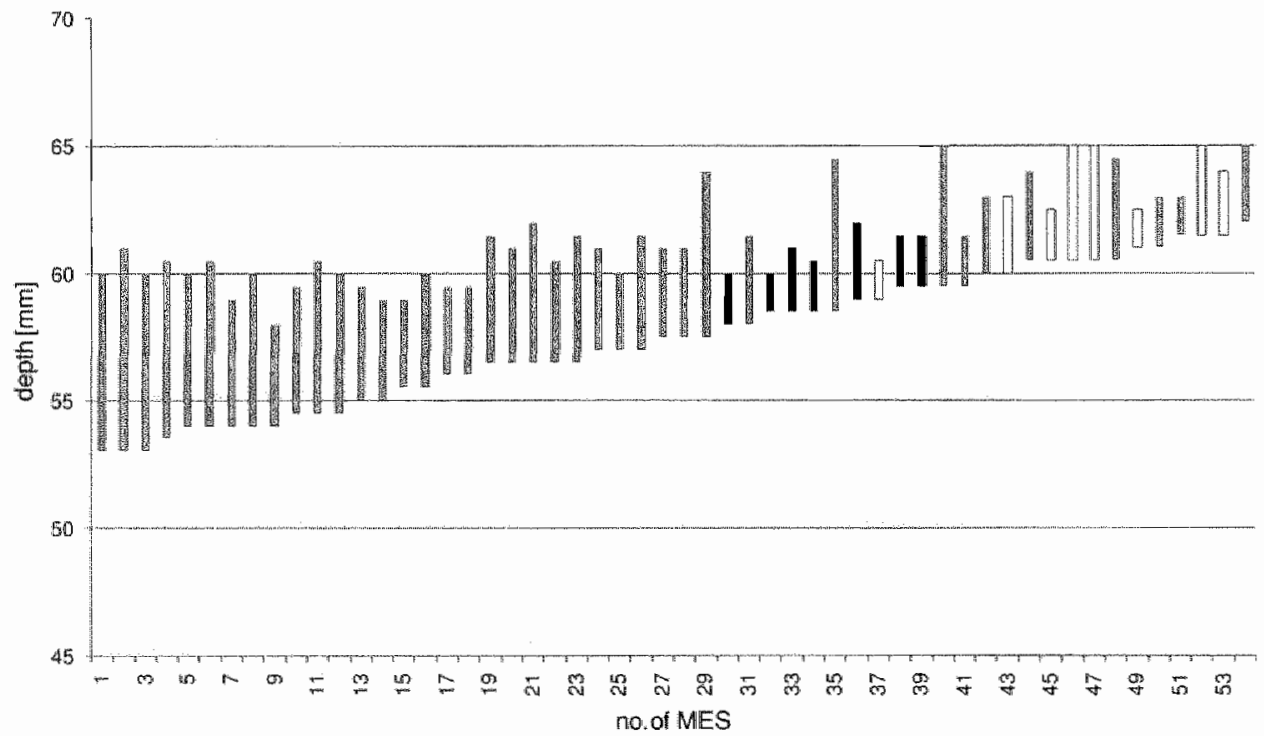

Figure 6 and $b$

Depth range of gaseous MES fitom both patients is given on the $y$-axis. Black bars indicate MES that showed a change of direction. and white bars those moving away from the transducer. Gray bars indicate all other MES. On the $x$-axis, the identification number of the MES is given. 


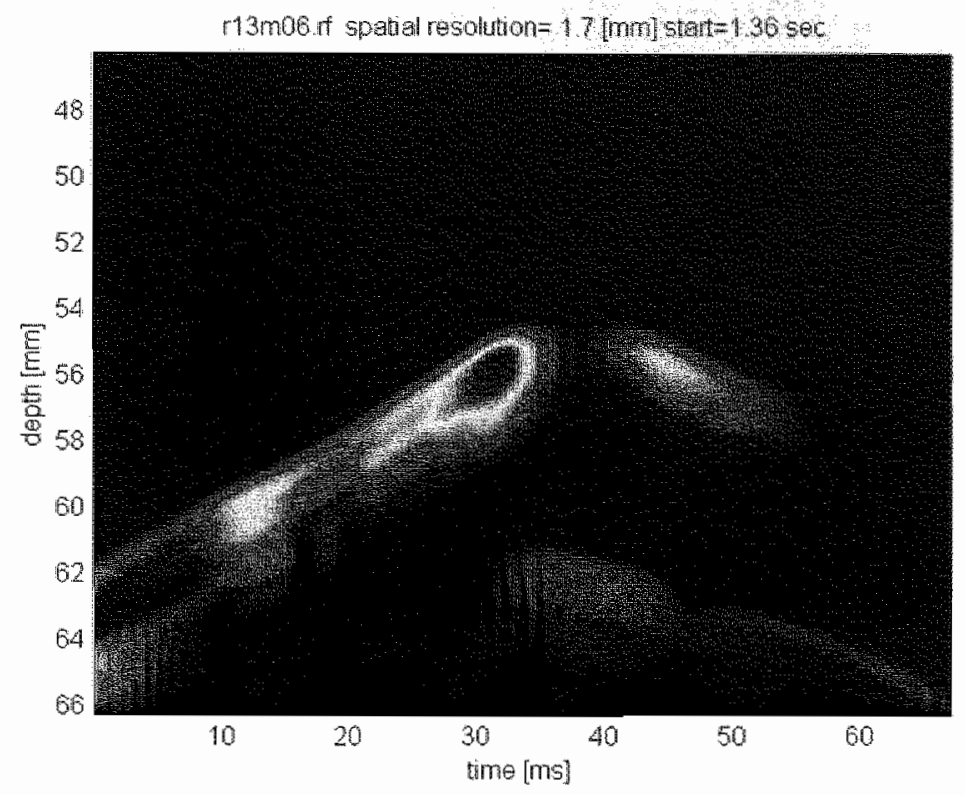

Figure 7 ia

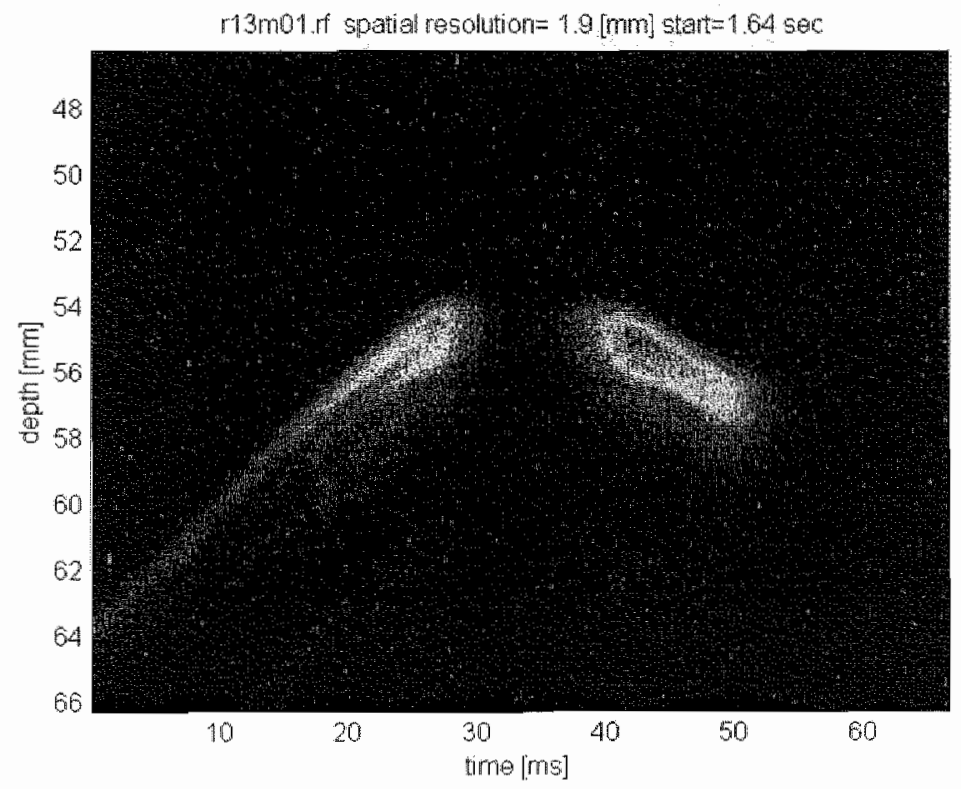

Figure 7a and $b$

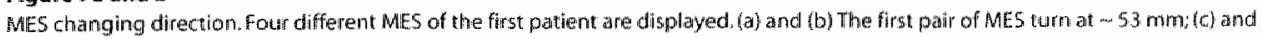
(d) the second pair at $\sim 60 \mathrm{~mm}$. Note that, at the turning point itself mo MES is visible. Presumably, this is attributable to the angle between US beam and motion direction at that distinct point, in combination with the clutter filter appliad 
Figure $7 c$
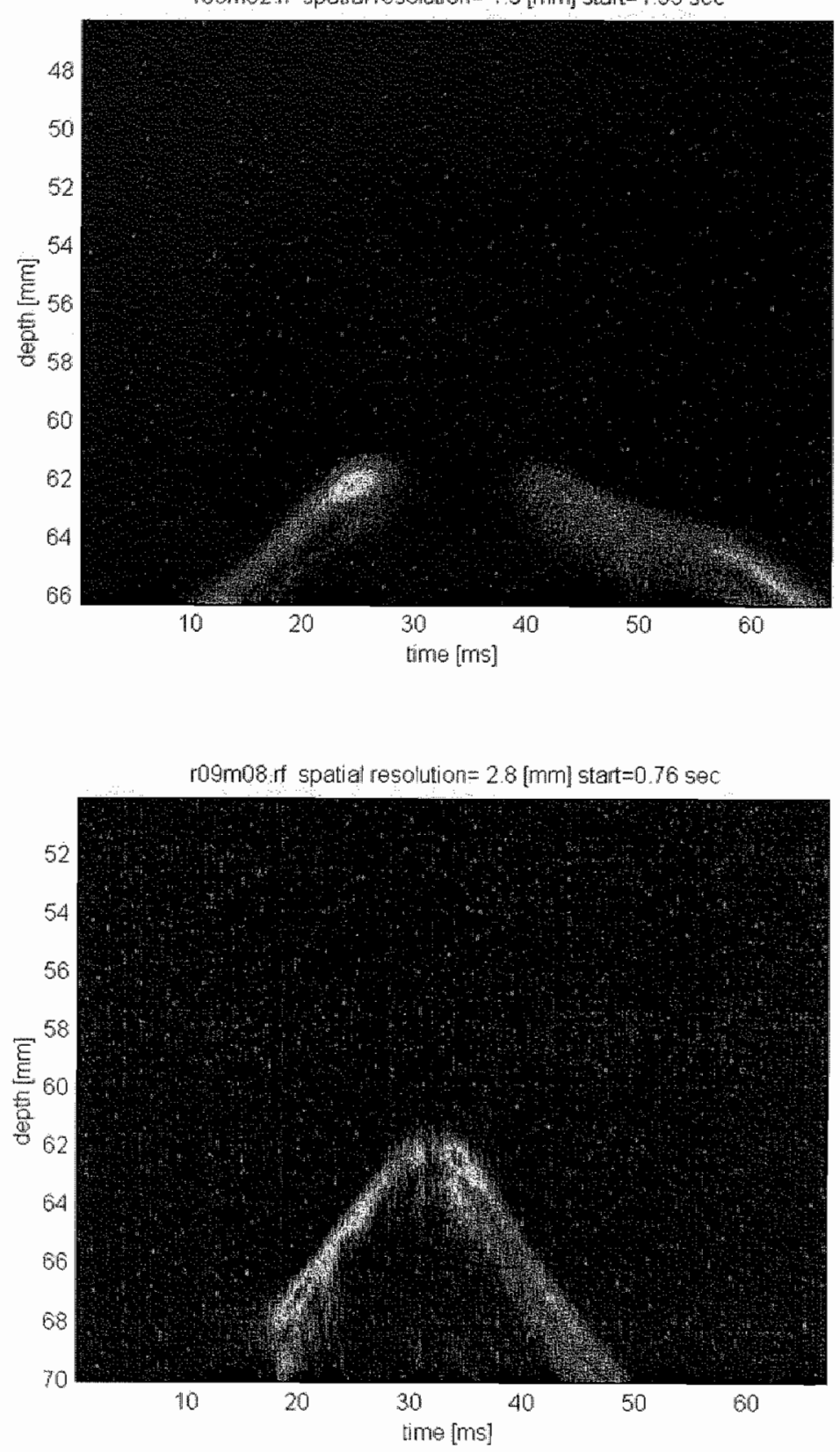
Figure 8 a
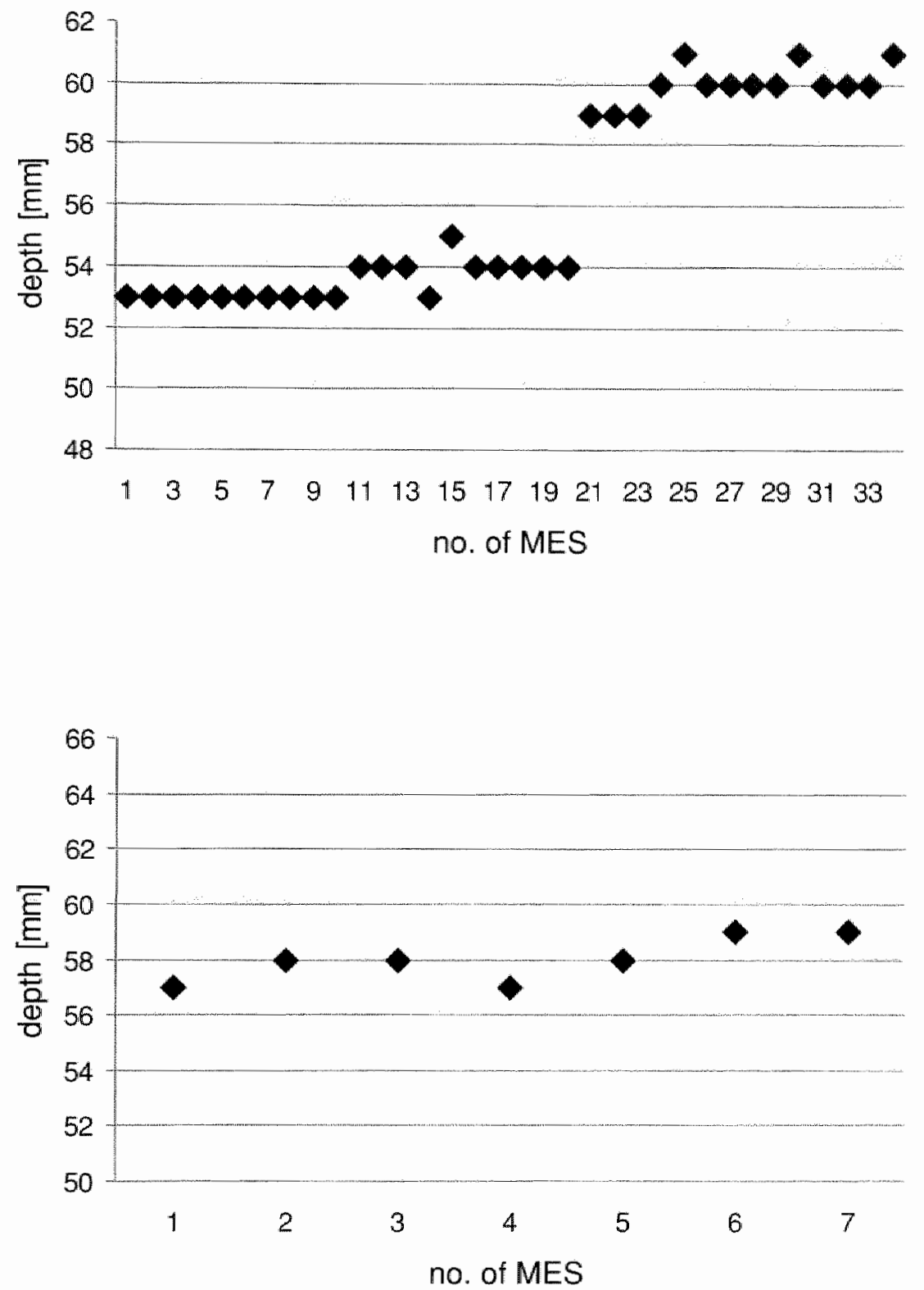

Filigure 8

Depth valties of "turning point $5^{\text {"in }}$ gaseous MES. The deptllwalues at which the direction of the gaseous MES from both patients changed is given on the $y$-axis in mm distance from the transducer. Note the consistent pattern with (ia) two narrow defth ranges in the first pattertit and (b) one in the second patieint. 


\section{Particulate MES}

As with conventional MES analysis, the SNR was considerably lower for this group of signals.

Despite median filtering of the signal, as described above, we could not determine the direction or, hence, velocity of 15 of 52 signals because they appeared, not as straight lines, but as circular or complex structures in the amplitude plot (Fig. 3).

Despite these restrictions, the anallysis of particulate MES revealed a distinct pattern in terms of depth ranges (all 52 MES) that was even more consistent than that seen for gaseous MES (Fig. 9). The fength over which the MES were present was comparable to that of the gaseou: MES $15.1 \mathrm{~mm}$ $\pm 2.1 \mathrm{~mm}$ ). The particulate MES did not exhibit motion away from the probe nor an abrupt change in direction.

\section{Figure 9a}

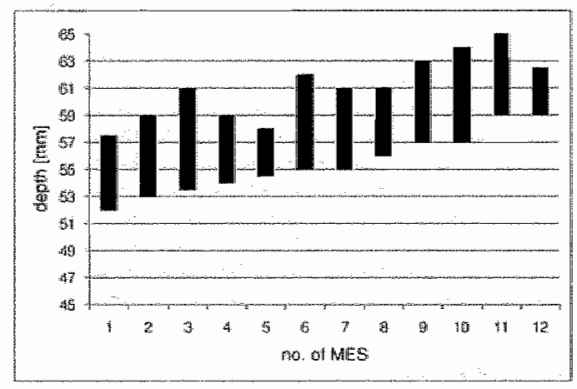

c

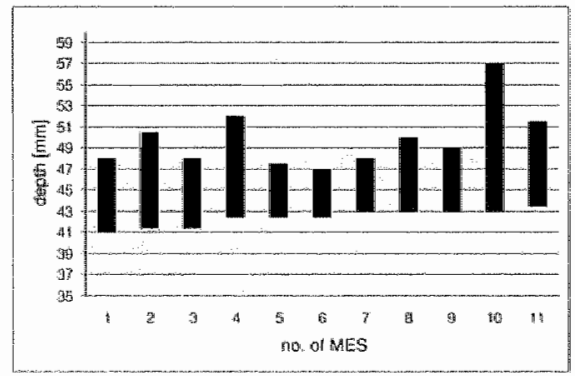

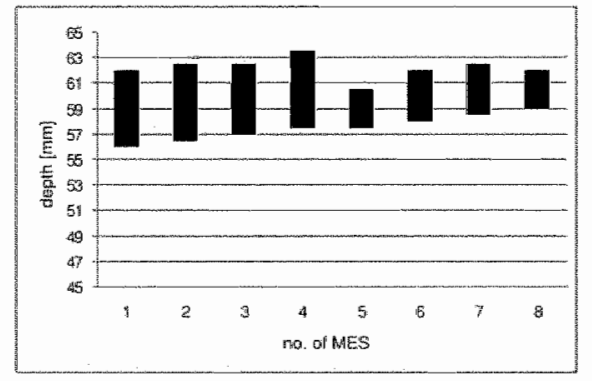

b

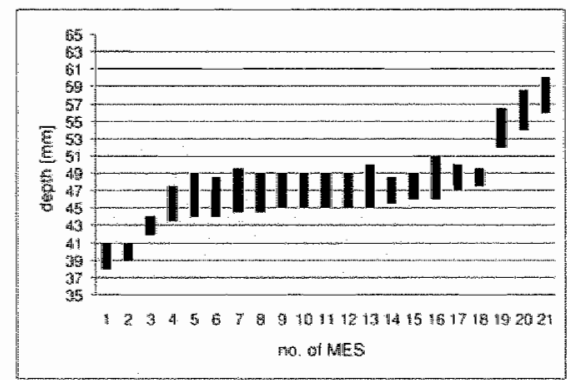

Figure 9a-d

Depth ranges of particulate MES from (a) to (d) the four patients are given on the $y$-axis in mon distance from the transclucer surface. On the $x$-axis, the ideratifictiontion number of the MES is giwen. 
DISCUSSIOn The system of MES evaluation presented here is based on the digitization and storage of unprocessed RF signals, and retains the temporal and spatial MES characteristics. It is characterized by a high spatial resolution on the order of 2 to $3 \mathrm{~mm}$, rather than 2 to $3 \mathrm{~cm}$, as is common for conventional TCD Doppler systems. Moreover the location at which the MES appear and vanish can be ascertained within a fraction of a $\mathrm{mm}$ (Fig. 2). This approach has a better spatial and temporal resolution than an online system described by Moehring and Spencer $(2002)$, which is based on $M$-mode. It has 33 sampling gates with a length of $6 \mathrm{~mm}$ at $2-\mathrm{mm}$ intervals, allowing for tracking MES and observing a change of flow direction. The MES are displayed as increased untensities superimposed on the power M-mode Doppler signal of the cerebral blood flow in the large intracranial arteries. The present approach emphasizes the temporal and spatial behawior of emboli, rather than their direct relationship with the velocity and amplitude of the surrounding bloodstream. Accordingly, the processing and evaluation are executed off-line to retain especially, the temporal behavior. So, it remains to be shown how the approach presented here will perform in an online system.

Four arguments support the assumption that we could trace the MES along the major cerebral vessels with a relatively small spatial error. First, the depth range of the MES was within a narrow range and the MES were considerably shorter and less variable than those reported from conventional system data analysis ( 5 mith et al. 1997a, 1997b). The findings concerning the depth range did not differ between gaseous and particulate MES, which underlines the reliability of the system in terms of correct MES localization. However, this also implies that the system proposed here is not suited to differentiate MES based on their composition. Second, with one single exception, all MES showed a rather constant velocity in the amplitude plat. So, the previously reported variability of MES velacity (Smith et al. 1997b) is unlikely to be evoked by velocity changes in the main stem of the MCA, but could be attributable to the spatial course of the MES in more proximall and distal vessel segments. Third, in case the MES only showed a movement away from the probe, the depth of initial appearance varied only by 1 to $2 \mathrm{~mm}$, suggesting that these MES entered the anterior cerebral artery. The fourth argument is the consistent depth where about a quarter of all gaseous MES changed direction. it is wery unlikely that this is an artefact, because the velocity and the depth range of both parts of the course were not identical (i.e., the MES did not appear symmetrically in the amplitude plot). The fact that, at the point of direction change, no intensity increase was visible is attributable to the angle between US beam and motion direction at that distinct point in combination with the clutter filter applied.

These findings suggest that the MES first were measured in the proximal segment of the MCA or the distal part of the internal carotid artery and then entered a large M2 branch with, at least initially, a flow away from the probe (turning point $\sim 53 \mathrm{~mm}$ ) or the anterior cerebral artery (turning point $\sim 58$ or $59 \mathrm{~mm}$ ). These observations are in agreement with earlier reports of the so-called "tail-sign." Furui et al. (1999) also hypothesized that a change of the spectral intensity was consistent with an MES abruptly changing direction. 
The observations presented here are consistent with the results of Smith et al. (1997b) describing rapid frequency modulations only with gaseous emboli. It is likely that a change of direction of an MES causes, for a short duration, a low frequency part in the middle of an MES as a consequence of the relative position to the US beam. The time domain data of the depth range containing the flow direction change, indeed, show a pattern that has been described by Smith et al. (1997b) (see Fig. 10). Therefore, the phenomenon described seems to differentiate between MES of gaseous and particulate nature, but it has to be kept in mind that only $25 \%$ of the gaseous MES of our study and $43 \%$ of the MES analyzed by Smith et al. (1997b) were positive. Furthermore, this effect is likely to depend on the individual anatomic situation.

All gaseous MES could reliably be discriminated from the Doppler background and described in terms of direction and velocity, but about a quarter of all particulate MES only appeared as round or complex structures. So, for considerable portion of particulate MES, there was no movement detectable in the amplitude plots, presumably due to the known lower SNR of these MES. We did not calculate an "embolus-to-blood ratio" to identify possible MES because signal acquisition was initiated by aural evaluation. Probably, the evaluation of particulate MES could be improved by considering a running envelope of the amplitudes of successive incoming RF signals or by increasing the US burst length.

The dual or multigate technique not only yielded a high variability in the signature of the same MES in two consecutive sample wolumes, but also resulted in "missing emboli" (i.e., the MES did not appear in the more distal sample volumel, which was interpreted as dissolving MES or MES vanishing into MCA branches (Molloy and Markus 1996; Georgiadis et al. 1998). Even "appearing MES" have been reported (Georgiadis et al. 1996) (i.e., MES only dletectable in the mare distal sample volume). The problem of MES appearing in only one of the two serially placed sample volumes cannot be answered on the basis of our data, but will be the subject of further research.

Conclusions In conclusion, the use of a customized digital postprocessing system based on the analysis of the RF signals allowed for a description of MES with a high spatial resolution. In contrast with the results obtained from conventional Doppler systems, both gaseous and particulate MES showed consistent patterns of MES signature. The previously reported variability of MES in terms of depth range and MES length is an artefact caused by standard Doppler processing of the relatively large MES amplitudes, and by the position and size of the sample volumes. 


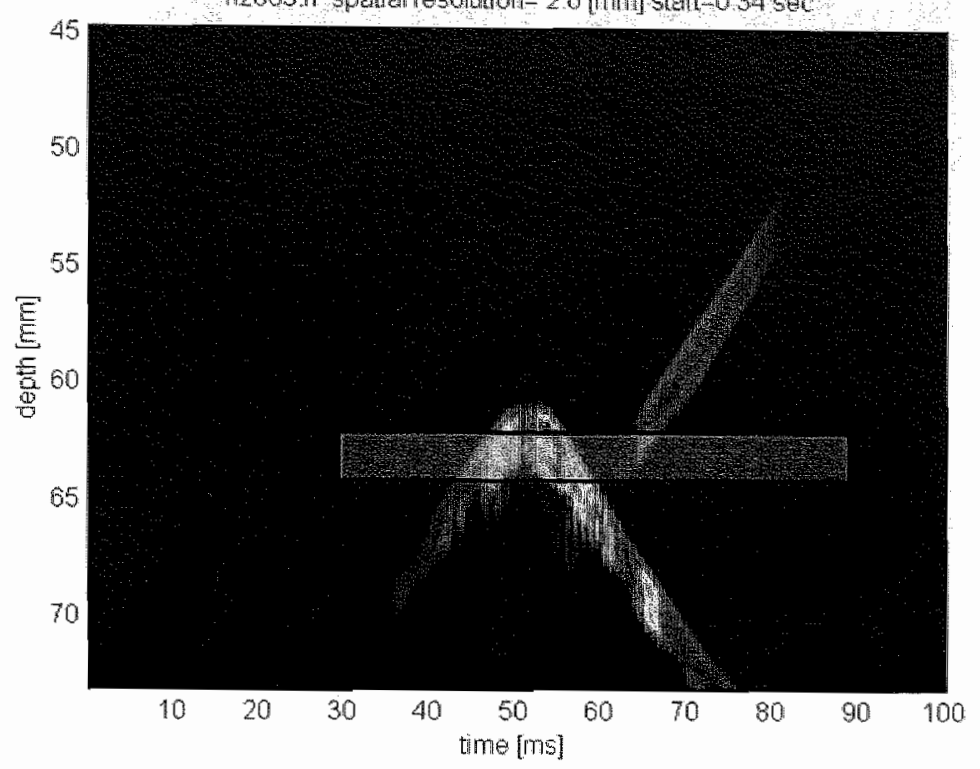

Figure 10a

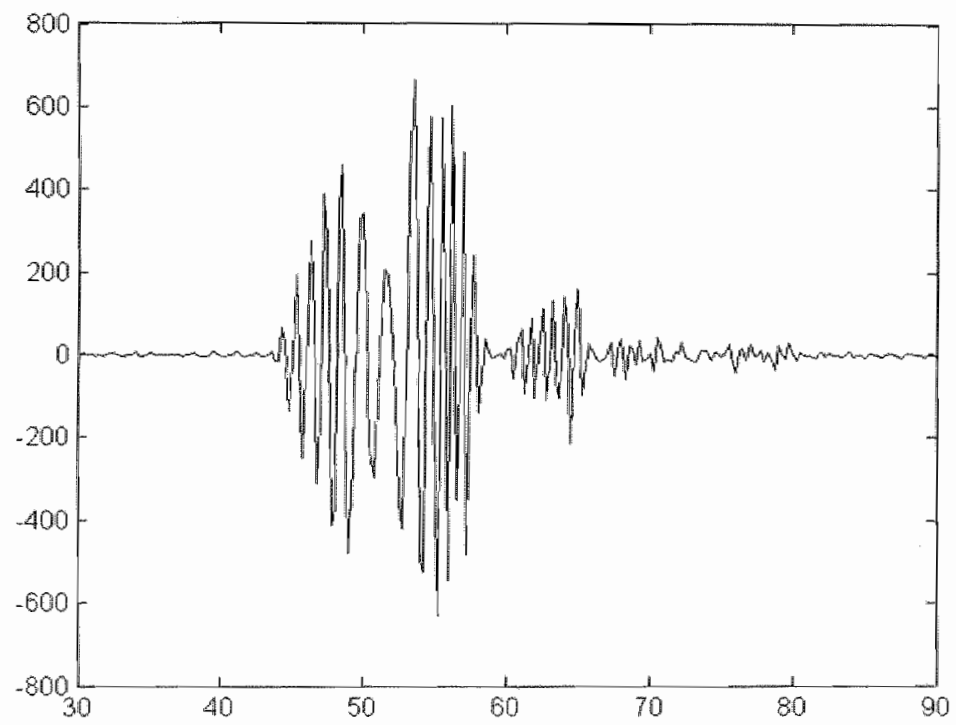

\section{Figure $10 \mathrm{a}$ and $\mathrm{b}$}

Complex MES: amplitude plot and time domain data. Two MES are present in laj the amplitude plot. The first, appearing at - 35 ms changes flow direction at $-50 \mathrm{~ms}$; the second appears at $\sim 60 \mathrm{~ms}$ and exhibits no change of flow direction. (b) The time domain clata a depth of $62 \mathrm{~mm}$. Taking a spatial resolution of $2 \mathrm{~mm}$ into account, the red bar in (a) indicates the depth fange of the diata in (b). Note that the the domain data 5 uggest a considerable change of MES welocity at $\sim 50$ ms that, obwiouslyt ts caused by the change of MES flow direction. Additionally, is not obvious from the time diomain wata how many MES contribute to the signal, whereas the amplitudte plot clearly depicts two MES. 


\section{References}

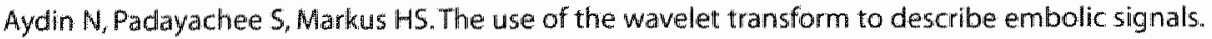
Ultrasound Med Biol 1999:25:953-958.

Biranids PJ, Hoeks APG, Willigers JM, Willekes C, Reneman RS. An integrated system for the noninvasive assessment of vessel wall and hemodynamic properties of large arteries by means of ultrasound. Eur J Ultrasound 1999;9:257-266.

Droste DW, Hagedorn G, Nötzold A, Siemens H.J, Sievers HH, Kaps M. Bigated transcramial Doppler for the detection of clinically silent circulating emboli in normal persons and patients with prosthetic heart valves. Stroke 1997;28:58:8-592.

Fan $L$, Evans DH. A real-time and fine resolution analyser used to estimate the instantaneous energy distribution of Doppler signals. Ultrasound Med Biol 1994;20:445-454.

Furui E, Hanzawa K, Ohzeki H, Nakajima T, Fukuhara N, Takamori M. "Tail sign" associated with microembolic signals. Stroke 1999;30:863-866.

Georgiadis D, Goeke $\mathrm{J}$, Hill $\mathrm{M}_{\mathrm{n}}$ König $\mathrm{M}_{*}$ Nabovi DG, Stögbauer $F_{\text {, Zunker }} \mathrm{P}_{\mathrm{z}}$ Ringelstein EB. A novel technique for identification and Doppler microembolic signals based on the coincidence method; in vitro and in vivo evaluation. Stroke 1996;27:683-686.

Georgiadis D, Wenzel A, Zerkowski HR, Zierz $S_{r}$ Lindner A. Automated intraoperative detection of Doppler microembolic signals using the bigate approach. Stroke 1998;29:137-139.

Markus. HS, Reid G. Frequency filtering improves ultrasonic embolic signal detection. Ultrasound Med Biol 1999:25:857-860.

Mess WH, Titulaer BM, Ackerstaff RGA. Middle cerebral artery anatomy and characteristics of embolic signals - a dual gate computer simulation study. Ultrasound Med Biol 1999:25:531-539.

Mess WH, Titulaer BM, Ackerstaff RGA. A new algorithm for off-line automated emboli detection based on the pseudo-Wigner power distribution and the dual gate TCD technique.

Ultrasound Med Biol 2000;26:413-418.

Moethring MA, Spencer MP. Power M-mode Doppler (PMD) for observing cerebral blood flow and tracking emboli. Ultrasound Med Biol 2002;28:49-57. 
Molloy $J_{r}$ Markus HS. Multigated Doppler ultrasound in the detection of emboli in a flow model and embolic signals in patients, Stroke 1996:27:15:48-1552.

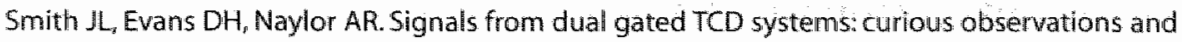
possible explanations. Ultrasound Med Biol 1997a:23:15-24.

Smith $J L$, Evans DH, Naylor AR. Analysis of the frequency modulation present in Doppler ultrasound signals may allow differentiation between particulate and gaseous cerebral emboli.

Uitrasound Med Biol 1997b;23:727-734.

Smith JL, Evans DH, Bell PRF, Naylor AR. A comparison of four methods for distinguishing Doppler signals from gaseous and particulate embolli. Stroke 1998;29:1133-1138. 
0

The depth of microembolic signal direction change corresponds with vessel anatomy

Submitted: Stroke 

Abstract Analysis of microembolic signals (MES) suggests a change of flow direction (CFD). The aim of the present study was to relate MES direction in an amplitude plot based on the radiofrequent (RF) signal to the vascular anatomy as seen with transcranial color coded duplex (TCCD). In five patients undergoing heart valve surgery or aortic arch replacement, preoperatively TCCD of the distal part of the internal carotid artery and the middle and anterior cerebral arteries on the right side was performed to determine potential depths of changes in flow direction. Peroperatively, a transcranial pulsed Doppler (TCD) monitoring probe was fixed over the right temporal bone. A customized RF based system, connected to the TCD device, captured and stored the MES. Offline, the color coded amplitude of the clutter filtered RF signals was plotted as a function of time (sample interval $0.17 \mathrm{msec}$ ) and depth (sample interval $0.05 \mathrm{~mm}$ ). A total of $313 \mathrm{MES}$ were recorded with 66 MES (21\%) in 4 patients showing a CFD. All MES with CFD could be assigned to maximally three different depth values, six out of eight CFD depth values as seen with the RF analysis were within one mm from a turn in flow direction as estimated with TCCD. A. CFD of MES occurred at a very limited number of dlepths and corresponded mostly with the intracranial wascular anatomy, namely a turn of the flow direction in the intracraniall vessels as abserved with TCCD. 
Introduction Microembolic signals (MES) in the middle cerebral artery (MCA) can be recognized by means of transcranial Doppler (TCD) sonagraphy. Although this method is now used for perioperative monitoring during carotid endarterectomy (Ackerstaff et al. 2000, Naylor et al. 2000), fundamental aspects of emboli detection are still unclear. Not only the clinical impact of MES in several patient groups is questionable (Mess and Hennerici 2001), but also the MES itself still poses questions. One of these questions was the meaning of the so-called "tail-sign" first described by Furui et al. (1999) in a subgroup of MES. These MES in the fast Fourier transform (FFT) typically is followed by a small signall with a reversed direction. In the time domain, the signal consists of two intensity increases with a very short time interval in between. These signals theoretically could have been evoked by an embolus moving successively towards and away from the probe. The same authors later supported this idea by an in vitro model (Furui et al. 2000). Further evidence for this theory was provided by the use of a power M-mode Doppler system (Maehring and Spencer 2002).

These authors described an example of an MES that first was related to flow towards the probe and subsequently to flow away from the probe. Mess et al. (2002), applying an amplitude plot of the radio frequency $(R F)$ signal, also found that a subgroup of MES showed a signature compatible with a change of flow direction (CFD). Since this RF based system gives information on MES characteristics with a high spatial resolution, a number of specific depth values could be distinguished at which a CFD occurred. The aim of the present study was to elucidate whether the MES showed a CFD corresponding to the vascular anatomy as seen with transcranial color coded duplex (TCCD). 


\section{Materials and Methods}

\section{Evaluation of intracranial vessel anatomy}

Five patients, scheduled for heart valve surgery or aortic arch replacement were enrolled in this study. Preoperatively, a TCCD examination of the distal part of the internall carotid artery (ICA) and the middle (MCA) and anterior cerebral arteries (ACA) on the right side were performed to determine potential depths of changes in flow direction. The vessels were visualised with a broadband phased array transducer (2-4 MHz; Hewlett Packard; Sonos 5500) via the transtemporal approach in the horizontal plane. Special attention was paid to the depth of the bifurcation of the ICA into the MCA and the ACA, as well as the anatomical configuration of large vessels branching off from the MCA. Power Doppler mode was applied to facilitate the identification of smaller vessels. Images were stored digitally and analyzed offline by one of the authors not aware of the results of the MES analysis. Figure 1a shows an example of the depth estimation of the flow direction change at the transition from the ICA into the ACA.

\section{MES sampling and analysis}

For the acquisition and description of MES a customized RF based system was connected to the TCD device (modified Multidop X 4; DWL, Uberlingen, Germany). The RF system has been described in detail elsewhere (Mess et al. 2002). In short, the RF signals were sampled on an external computer connected to the TCD device. RF sampling was synchronously with the emission trigger (pullse repetition frequency $\sim 6 \mathrm{kHz}$ ) at $16 \mathrm{MHz}$ with a dynamic range of $72 \mathrm{~dB}$. The actual spatiall resolution, mainly depending on the length of the emitted ultrasound bursts (3-4. $\mu \mathrm{s}$ ), was on the order of 2-3 $\mathrm{mm}$. If an MES was acoustically noticed, a button was pressed and the RF signals within one second before and after the MES (depth range $20-30 \mathrm{~mm}$ ) were captured and stored on harddisk. All MES were sampled in the final phase of the monitoring procedure, when the heart was beating again. This perlod is characterized by a large number of air bubbles, so, the MES used for further analysis were assumed to be gaseous. Both, the preoperative TCCD examination and the peroperative TCD monitoring were part of our routine monitoring procedures in this patient group.

offline, 128 point FFTs were calculated, wisualizing both the background Doppler spectrum and the MES. From the FFT plat, which covered the complete sample $(2-35)$, the appropriate time window $(\sim 50-100 \mathrm{~ms})$ containing the MES was chosen for amplitude plots, The color coded amplitude of the clutter filtered RF signals was plotted as a function of the (sample interval 0.17 msec) and depth (sample interval $0.05 \mathrm{~mm}$ ). An MES appears typically as a line of increased intensity in a two dimensional amplitude plot. A CFD was observed if an MES appeared first as a line of increased intensity towards the probe and consecutively as a line away from the probe. The depth of a CFD was visually estimated. Figure $1 b$ shows an MES with a CFD in the FFT plot and figure 1c shows the same MES in the RF amplitude plot. 
ReSUlTS Four out of five patients had MES with a CFD. In total, 313 MES were recorded in these four patients and 66 MES (21\%) showed a CFD. Taking a measurement error of $2 \mathrm{~mm}$ into account, all MES with CFD could be assigned to maximally three different depth values, irrespective of the number of MES per patient. Six out of eight CFD depth vallues occurred within one $\mathrm{mm}$ from a turn in flow direction as estimated with TCCD. Table 1 gives an overview of the number of MES that showed a CFD and the depth values of both the TCCD and the RF amplitude plots.

\section{Tablle 1}

Overview of microembolic signals and changes of flow direction

\begin{tabular}{|c|c|c|c|c|c|c|}
\hline & $\begin{array}{l}\text { MES analyzed } \\
\text { (n) }\end{array}$ & $\begin{array}{l}\text { MES with CFD } \\
\text { [n] }\end{array}$ & $\begin{array}{l}\text { MES with CFD } \\
{[\%]}\end{array}$ & $\begin{array}{l}\text { depth of CFD [mm] } \\
\text { RF analysis }\end{array}$ & $\begin{array}{l}\text { depth of CFD [rmm] } \\
\text { tc-duiplex }\end{array}$ & $\begin{array}{l}\text { matching } \\
\text { CFD values }\end{array}$ \\
\hline patient 1 & 80 & 19 & 24 & & & \\
\hline depth of CFOt & & 6 & & 60 & 61 & $x$ \\
\hline depth of CFD2 & & 4 & & 55 & & \\
\hline depth of $\mathrm{CFD}_{3}$ & & 9 & & 49 & 50 & $x$ \\
\hline depth of $\mathrm{CFO} 4$ & & & & & 44 & \\
\hline patient 2 & 76 & 9 & 12 & & & \\
\hline depth of $\mathrm{CFO}$ & & 2 & & 58 & 58 & $\mathrm{x}$ \\
\hline depth of $\mathrm{CFO} 2$ & & 5 & & 52 & 52 & $\mathrm{x}$ \\
\hline depth of $\mathrm{CFO3}$ & & 2 & & 45 & & \\
\hline patient 3 & 79 & 36 & 46 & & & \\
\hline depth of CFOH & & 36 & & 62 & 63 & $x$ \\
\hline depth of $\mathrm{CFO} 2$ & & & & & 53 & \\
\hline patient 4 & 72 & $a$ & 0 & & & \\
\hline patient 5 & 6 & 2 & 33 & & & \\
\hline depth of CFDI & & 2 & & 55 & 56 & $x$ \\
\hline sum & $3 \| 3$ & 66 & 21 & $\mathbf{8}$ & 8 & 6 \\
\hline
\end{tabular}

In five patients a total of 313 microembolic signals (MES) were evaluated. Sixty-six MES showed a change of flow direction (CFD) with the radiofrequency based alzalysis. Maximally, three different depth values were found in an individual patient (fifth columin). The $\mathrm{CFO}$ depths as seen with transcranial color coded duplex are given in column 6 , while matching depth values of the wo methods (6/B) are marked (x). 


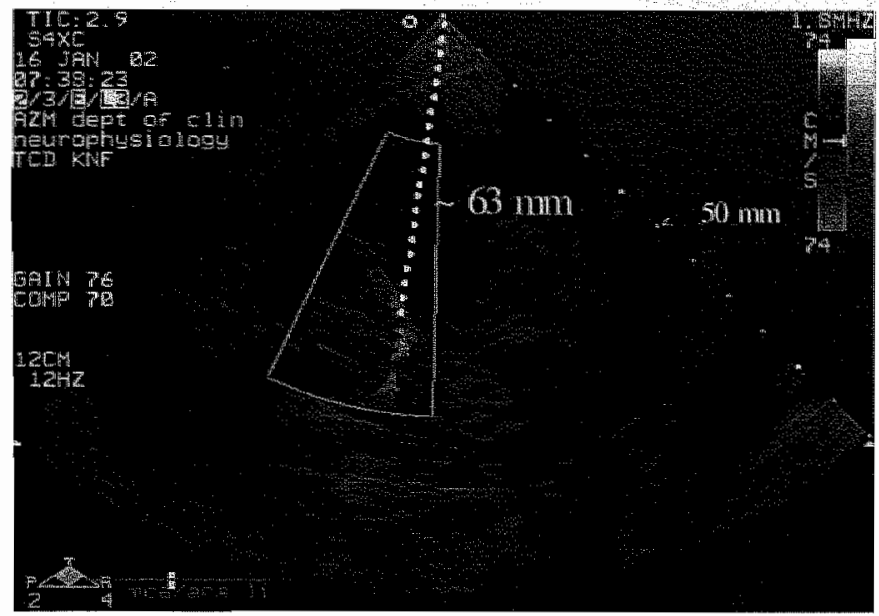

Fïgure 1a:
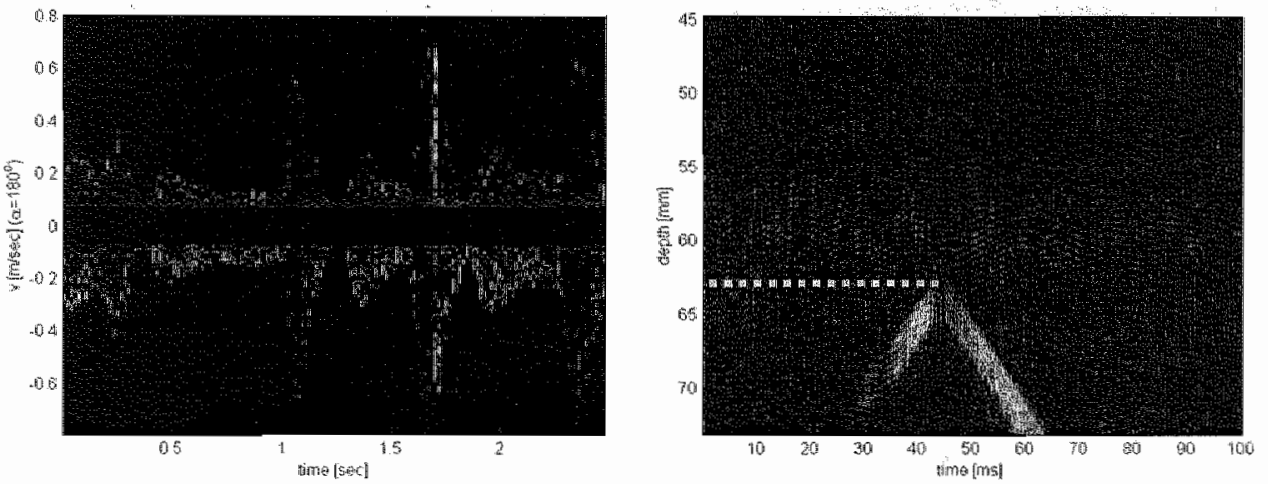

b, c

Figure $1 \mathrm{a}, \mathrm{b}$ and $\mathrm{c}$

Example of an MES with a change of flow direction in the FFT and the RF amplitude plat and the conresponding TCCD ingage The TCCD image (a) shows the MCA and the ACA in the horizantal piane. At a depth of approximately 63 mm, the very distall part of the ICA splits into the MCA and the ACA. So, a part of the flow from the distal ICA, which is directed towards the probe and color coded un red will abruptly change direction and then flow away from the probe in the ACA which is color cocled in blue.

At depth ranges between 60.1 and $69.3 \mathrm{~mm}$ the Fast-Fourter Transform (t) shows a bidirectional Doppler signal, since the MCA and the ACA are both within the sanple wolume. At about 1.75 an MES is visible as a short lasting high intensity signal, coded in red and yelliow. Despite the rather low temporal resotution of this techricue, the MES first seems to move towards the probe and subseguently away from it.

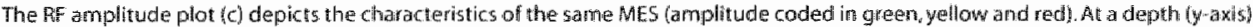
of about $70 \mathrm{~m} m$, the MEs first appears and moves $(30-45 \mathrm{~ms} ; \mathrm{x}$-axis) lowards the probe. At a depth of about $63 \mathrm{~mm}$, however, the intensithy increase for a very shot rime is absent and then moves away from the probe (45-65 ms). So, this MES displays a change of flow direction at a depth of about $63 \mathrm{~mm}$. 
DiSCUSSion Using a high temporal and spatial resolution technique, we showed that about $1 / 5$ of the gaseous MES sampled at the finall stage of cardiac or aortic arch surgery displayed a pattern compatible with a CFD. Irrespective of the number of MES in an individual patient, the depths at which a CFD occurred showed a marked consistency. Seventy-five \% of the CFD depth values were in good agreement with the wascular anatomy as evaluated with TCCD. These findings underline that the RF signal based analysis of MES by means of an amplitude plot provides detailed information with a very high resolution in space and time.

Two CFD wallues as seen with the RF based analysis were not seen on TCCD. This might be explained by the fact, that TCCD was performed only in the horizontal plane. Possibly, insoniation also in the vertical plane would have shown additional vessels branching off the MCA and giving rise to a change of flow direction. Furthermore it has to be considered, that two different ultrasound systems were applied, which is likely to introduce inaccuracies when the data are compared with each other.

The coincidence of the CFD values from TCCD and MES analysis confirms the suspected relationship between vascular anatomy and MES signature (Furui et al. 1999, Mesis et al. 2002). Furuil et al. (1999, 2000) discussed the relationship between the MES and the tail sign in the time domain data, when two serially placed sample volumes were used. These authors reported that characteristically the time delay between the MES and the tail sign was shorter in the more superficially placed sample volume. Our data explain what the basis for this observation is and figure 2 shows an example. The time domain data can be evaluated for two different depth ranges (fig $2 a$ ), matching the duration of the emitted ultrasound burst which corresponds to the sample volume of conventionall Doppler systems. As is obvious from the amplitude plot, the MES will appear two times in the time domain data of sample volume " $\mathrm{b}^{\prime}$ (fig $2 \mathrm{~b}$ ) and ' $c$ ' (fig 2c). The movement of the MES towards and consecutively away from the probe causes two successive amplitude increases. The time delay between the two amplitude increases is longer for a deeper depth. Since both intensity increases are caused by the same MES, it is, however, not correct to refer to the MES and the subsequent taill as separate signal entities.

In our patient group, the overall incidence of MES showing a CFD was about $20 \%$, which is comparable to the data of UhImann et al. (2000) who also reported on the tail-sign in about $10 \%$ of MES measured in patients with prosthetic heart valves. The incidence of the tail sign was on the same order as in the work of Furui et al. (1999), who also considered patients with artificial heart valves. Uhlmann et al. (2000) never encountered arrefacts with a tail sign. So, the presence of a tail sigm has a low sensitivity, but is very specific for MES.

Smith et al. (1997) reported on sudden frequency modulations of MES. They defined several types of frequency changes in the time domalin data. Their type III pattern ("rapid decrease in frequency that quickly returns to the frequency present before the sudiden decrease") cain be reproduced by our data (see figure 2b). So, it might be, that Smith et al. (1997) also observed a CFD of MES. Interestingly, Smith et al. (1997) exclusively found this pattern in gaseous MES. Since we also detected a CFD only in gaseous MES (Mess et al. 2002), the question arises why particulate MES do 


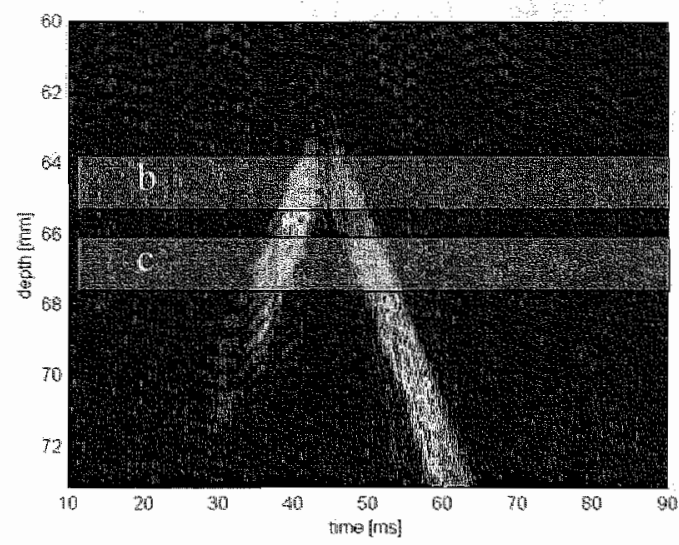

Filgure 2a

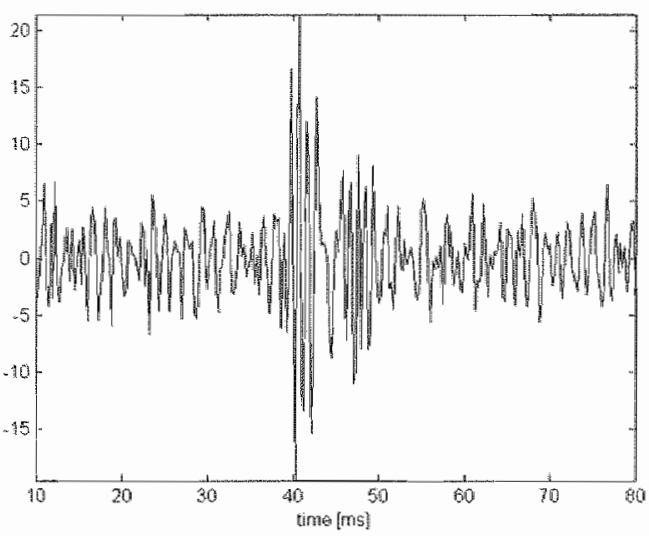

b

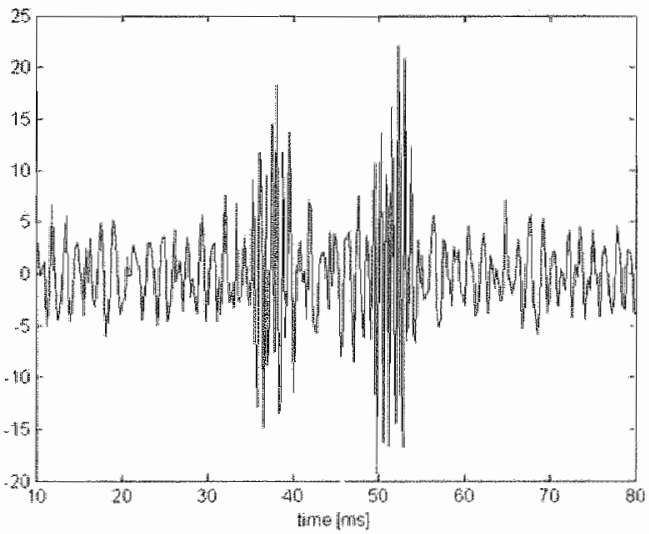

Figure 2a, b and $c$

The relation between the amplitude plot and time domain data at different depths. The amplitude plat (a) shows the same MES as

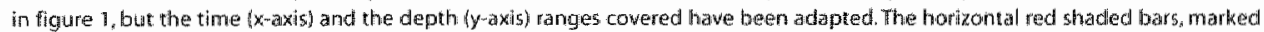
with "b" and " $c$ "indicate the depth ranges of the time domain data in figure $26(-64.5 \mathrm{~mm})$ and $2 \mathrm{C} / 66.5$ mm). The thickness of the bars $6 \times 1,6 \mathrm{~mm}$ ) reflects the actual spatial resolution of this example. The time domain data of a more superficlal depth thity show two intensity increases. which are separated by a very short and relatively low frequency segment. The time domain data of a deeper locatton (c) show a larger spacing between the two intensity increases stemming from the same Muss, firstly mowing towards and subsequently away from the probe. 
not show this patterns. Smith et al. (1997) speculated that gaseous MES might be more susceptible for external forces than particulate MES and hence could be more easily swapped to a bloodstream layer with a different velocity. Alternatively, the interaction between the ultrasound beam and the gaseous bubble was suggested as a possible mechanism for the phenomenon. Our data, however. show clearly the relationship between a CFD, the time domain data and the actual vascular anatomy. Since about $10 \%$ of the blood from the ICA will enter the $A C A$, it might be assumed, that also 10\% of the MES will show a CFD at the intracranial bifurcation, irrespective whether they are gaseous or particulate. So, the question why a CFD is exclusively observed for gaseous MES, is intriguing. Probably, their high reflectivity (echogenicity) is responsible for the detectability in relatively low sensitivity areas of the sample volume. An increase of the emitted ultrasound power and/or a higher sensitivity of the Doppler system possibly would provide better information on the pathway of particulate MES. The increase of the ultrasound power would be permitted considering the very short pulse length used ( 3 -4. us). So, the maximal output would not exceed the allowed limit of $500 \mathrm{~mW} / \mathrm{cm}^{2}$.

Our data support and refine the observations of Moehring and Spencer (2002) on MES first appearing in the ICA and consecutively in the ACA. The power M-made Doppler applied by Maehring and Spencer is characterized by an effective sample volume length of $6 \mathrm{~mm}$, whereas our system provides data with a spatial resolution on the order of 2 to $3 \mathrm{~mm}$. Furthermore, the power M-mode Doppler will display the color coded flow direction depending on the mean velocity of all signalls within a sample volume. Large sample volumes covering vessels with different flow directions will induce an uncertainty about the exact depth of the bifurcation of the ICA into the MCA and ACA.

In conclusion, with specific processing of the ultrasound RF signal it is possible to track MES in depth. A change of flow direction of MES occurred at a very limited number of depths and corresponded mostly with the intracranial vascular anatomy, mamely a turn of the flow clirection in the intracranial vessels as observed with TCCD. These findings confirm that it is possible to follow the course of MES in the basal cerebral arteries with an RF based acquisition system with a high spatial resolution. 


\section{References}

Ackerstaff RGA, Moons KGM, van de Vlasakker CW, Moll FL, Vermeulen FEE, Algra A, Spencer MP. Association of intraoperative transcranial Doppler monitoring variables with stroke from carotid endarterectomy. Stroke 2000;31:1817-1823.

Furui E, Hanzawa K, Ohzeki H, Nakajima T, Fukuhara $\mathbb{N}_{n}$ Takamori M. "Tall sign" associated with microembolic signals. Stroke 1999;30:863-866.

Furui E, Hanzawa K, Nakajima T, Fukuhara N, Komai K, Yamaida M. In vitro evaluation of the mechanism for "tail signs" associated with microembolic signals (Abstract). Cerebrowasc Dis 2000;10(suppl.1):2.

Mess WH, Hennerici MG. Highl Intensity Transient Signals. In: Hennerici M, Meairs S (eds). Cerebrovascular Uttrasound; Theory, Practice and Future Developments. Cambridge, Cambridge University Press, 2001, pp. 297-316.

Mess WH, Willigers JM, Ledoux LAF, Ackerstaff RGA, Hoeks APG. Microembolic sigmal description: A reappraisal based on a customized digital postprocessing system. Ultrasound Med Biol. 2002;28:1447-1455.

Moehring MA, Spencer MP: Power M-mode Doppler (PMD) for observing cerebral blood flow and tracking emboli. Ultrasound Med Biol 2002;28:49-57.

Naylor AR, Hayes PD, Allroggen H, Lennard N, Gaunt ME, Thompson MM, London NJM, Bell PRF. Reducing the risk of carotid surgery: a 7-year audit of the role of monitoring and quality control assessment. J Vasc Surg 2000:32:750-759.

Smith J, Evans DH, Naylor AR. Analysis of the frequency modulation present in Doppler ultrasound signals may allow differentiation between particulate and gaseous cerebral emboli. Ulltrasound Med Biol 1997;23:727-734.

Whimann F, Schulte-Mattler WJ, Georgiadis D. Postembolic spectral patterns of microembolic silgnals (Abstract). Cerebrovasc Dis 2000;10(suppl.1):3. 
Epilogue 

The prescence of microembolic signals (MES) as detected by transcranial Doppler (TCD) ultrasonography has been established as a risk factor for stroke or TIA in the context of carotid endarterectomy (CEA). Preliminary data also suggest that MES might indicate an elevated thromboembolic potential of a carotid artery plaque. The most important disadvantage of the relatively new ultrasound technique is the long-lasting monitoring session required, prompting a need for a reliable automatic MES detection system. However, the successful development of such a system has turned out to be surprisingly difficult, as compared with the ease with which the human observer can detect MES. This chapter will discuss the inter-relationship of the MES characteristics and their detectability with automatic MES detection systems. Special emphasis will be put on the moving nature of an MES. Finally the focus will be on the current status of MES detection for patient care.

Three automatic embolus detection systems, the first to be commercially avallable, were tested (v.Zuilen et al. 1996; chapter 2) against a panel of human experts and also compared to each other based on the same data. The systems had been reported to perform excellent (Siebler et al. 1994, Georgiadis et al. 1995, Brucher and Russell 1993, Markus and Loh 1993). Our evaluation, which was bassed on the analysis of MES stemming from carotid artery plaques, however, showed a marked difference in the agreement between the human observers and in agreement between human gold standard and the automatic systems. Whilst there was a very good agreement between the human observers with $\kappa$ values better than 0.95 , the $\kappa$ values for the agreement of the automatic systems only reached a level of $\sim 0.4$ to 0.5 , which is too low for clinical use. MES originating from carotid artery plaques mostly show a relatively lower intensity than MES caused by artificial heart valves. So, the former subgroup of MES appears to be the most difficult to identify "Cullinane, personal conversation, 2002). Another reason for the rather disappointing results of our analysis presumably was a different statistical appraach compared to that which was applied in earlier studies. Instead of counting all MES detected within a given time, we evaluated for every second, whether an MES was present (for the human observer) and whether the automatic systems detected the MES at that very time point.

Since our comparative study was performed, further developments in the field of automatic embolus detection based on a singlle sample volume took place. Different signal analytical approaches have been proposed, alike non-linear forecasting (Keunen et al. 1998), the so called "narrow band hypothesis" (Roy et al. 1998), fundamental tone analysis (v.Dijk et al. 2002) and parametric autoregressive modeling (Kouarné et al. 2002), which, however, did not result in a clinically relevant improvement compared to the systems examined earlier. A re-evaluation of one of these systems, based on a neural network (Kemeny et al. 1999) basically confirmed the results. of our evaluation (van Zuillen et al. 1996). The description of additional MES properties like the tail-sign (Furui et al. 1999) or other specific postembolic spectral patterns, (Ries et al. 1998) also could not contribute to an essentially improwed automatic recognition of MES. 
Only recently, a frequency filtering approach was proposed (Markus et al. 1999, Markus and Reid 1999), which basically analyses intensity increases within frequency bands of the composite Doppler signal. Compared to a human expert panel (Cullinane et al. 2000), this system performed comparably good and mean whille thas been incorporated into a commercially available system (PIoneer 8080; EME, Kleinostheim). in contrast to the three systems that had been compared by van Zuilen et al. (1996), the good performance of the frequency filtering approach could be confirmed by an independant research group (Munts, et al.2002). However, the good performance of this approach was obtained with MES detected after carotid endarterectomy. In asymptomatic patients with MES originating from carotid plaque formation, the performance was considerably worse with the consequence that the system is nat suitable for this patient group (Reihill et al. 2002). Because a Fourier transform -based spectral analysis is not appropriate for both temporal and spectral characterization of a short lasting intensity increase as an MES, the use of wavelet transforms was proposed (Aydin et al. 1999, Devyust et al. 2000). Contrasting to Fourier transforms, the wavelet transformation makes use of variable time windows. High frequencies are estimated with short and low frequencies with lang time windows. Theoretically, this will result in a better compromise between time and frequency resolution of the signal analysed (Devyust et al. 2000). A more advanced use of the wavelet transform is the so-called "matching pursuit", which basically makes the application of the wavelet transform more efficient (Devyust et al. 2000). The potential of MES characterization by means of the wavelet transform has been demonstrated by preliminary studies (Aydin et al. 1999, Devyust et al. 2000) $\|$ ts performance as an automatic embolus detection in combination with a dual-gate system will be discussed later. Another alternative for FFT is the Wigner distribution function and its derivations (Fan and Evans 1994). This will be discussed below in more detail.

Fan et al. (2001) proposed a "rule-based expert system" for the automatic detection of MES. This system illustrates how the experience of more than 10 years. MES research contributes to the development of a modern and complex approach. Different analyses of the same MES are performed in the frequency and time domain and a multitude of parameters is fed into a "blackboard evaluation" which resembles the neural network approach mentioned above. The use of a blackboard-type intermediate hypotheses and decision base incorporates "empirical or public knowledge", which is based on the human experience with MES detection and hence resembles the learning proces of a neural network. Preliminary data are promising even for low intensity MES, but the system has not yet been tested in an independent multicenter setup with different classes of MES (Fan, personal communication 2002).

Since automated MES detection with one sample volume (5V) only provided modest results, we performed a pilot study to evalluate the characteristics of MES when analyzed with two SVs placed serially behind each other (Mess et al. 1996 and Mess et al.1997). Two patients undergoing coronary artery bypass grafting were monitored using identical TCD settings.

The middlle cerebral artery (MCA) was insonated with two SVs (length of emitted bursts: $\sim 10 \mathrm{~mm}$; distance between the centres of the SVs: $10 \mathrm{~mm}$ ). In total 24.2 embolic signals were assessed as 
well as 250 artefacts. Special modified software was applied to measure manually the distance $(\Delta t)$ between the embolic signals (at the beginning and the end) in the wo SV 5 as seen in the time domain data. The criteria for embolic signals were the typical audible properties and an intensity increase of $4 \mathrm{~dB}$ above background. The mean $\Delta t$ of the embolic signals was 31 (SD 21) $\mathrm{ms}$ (start); and - 3 (SD 12) $\mathrm{ms}$ (end) in the first patient and 5 (SD 6) ms (start), and 2 (SD 5 ) ms (end) in the second patient. With respect to artefacts, particularly electrocautery also showed a $\Delta$ t (range: $0-310 \mathrm{~ms}$ ) depending on the settings of the TCD and electrocautery device.

We concluded from these preliminary data that the use of two serially placed sample volumes offers an additional facility for automated differentiation between emboli and most artefacts, but that there was an unexpectedly large variation of $\Delta t$ with even negative walues, i.e. the MES appearied earlier in the second than the first sample volume. These data were in good agreement with the observations of other authors (Georgiadis et al. 1996, Smith et al. 1996). Furthermore, electrocautery presented a major problem, causing signal intensity increases with unpredictable $\Delta t$ that eventually were in the typical range of MES. For the embolic signals, the variation of $\Delta t$ could depend on the acoustic properties of the embolus, the TCD settings, and the anatomy of the MCA. A computer model, allowing for changing TCD settings like the insonation angle and the sample volume properties as well as the MCA anatomy, indeed showed that only modest changes of the variables led to extreme wariations of the critical $\Delta$ t walues (Mess et al. 1999; chapter 3). In another computer model, the importance of the insonation angle for the different MES characteristics in both sample volumes applied has also been described ( 5 mith et al. 1997).

Also, other MES characteristics as seen in the time domain data varied considerably, including the amplitude as compared to the fictive Doppler background. Eventually, the amplitude increase in one of the sample volumes was such low, that in the in wivo situation with its vartable Doppler background intensity due to e.g. speckle, it can not be expected that these MES were detectable in both sample volumes. These "missing emboli" can be observed in a substantial number of MES (for example: Lindner et al.1997), if two sample volumes placed serially are aipplied.

Fan and Evans, as well as Smith et al. (1994 and 1995) had proposed the pseudo-Wigner distribution function (WDF) for the description of MES. In their off-line system a temporal resolution of 80 . 5 could be achieved, compared to 10-20 ms obtainable by means of a Fourier transform. Unlike FFT, the WDF is capable of a high temporal resolution without the expense of a low frequency resalution. We (Mess et al. 2000; chapter 4) applied this signal analysis technique for the calculation of the critical $\Delta t$ values in an off-line automated emboli detection system. For data acquisition, the two sample volumes were placed with a relatively small distance in between, the receiver gate time (see appendix) was different for both sample volumes. These TCD settings were derived from the computer simulation and theoretically should result both in reliable $\Delta t$ values within a narrow range and only a small number of missing emboli.

The results in terms of specificity and sensitivity were sufficient, if the MES was detected in both sample volumes. However, approx. 1/4 of all MES were "missing emboli", i.e. they could not be detected in the second sample volume and hence a $\Delta$ t measurement was not possible. 
This phemomenon might also explain the modest performance of another automated dual-gate approach (Droste et al. 1997, Georgiadis et al. 1998., although the authors did nor specify how Many MES only appeared in one sample volume.

Recently, Devyust et al. (2001) evaluated a combined approach of the dual gate technique and a wavelet representation (the matching pursuit, see abovel. The achieved sensitivity and specificity let the authors conclude that the combined approach "reliably rejects artifacts from emboli". A sine qua non for a signal intensity increase to be accepted as an MES was a $\triangle t$ of more than 4 ms. Yet, their paper does not give any information on "missing emboli" or MES with a $\Delta t$ shorter than $4 \mathrm{~ms}$, which is very likely to occur and appeared to be the major drawback of our own study (Mess et al. 2000; chapter 4).

A variant of the dual gate technique was proposed by Georgiadis et al. (2000) and Brucher and Russell (2002). The second sample volume is intentionally placed very superficially and thus outside the middle cerebral artery ("arbitrary sample volume"). So, in the case of an MES in the first sample volume, no intensity increase is detectable in the superficial sample volume. Despite methodological problems (ultrasound beam characteristics in the near and far field are different), this method was reported to perform excellent in the case of gaseous MES. However, the results of the analysis of the more difficult to detect particulate MES from a "potential native arterial or cardioembolic source" indicated that this method does not represent an essential step forward towards a reliable automated MES detection (Georgiadis et all.2000). Brucher and Russell (2002) combined the "arbitrary sample volume" or "reference gate" with another new paradigm, the so-called "quarter Doppler shift". The middle cerebral artery is insonated simultaneously with 2 and $2.5 \mathrm{MHz}$ with the same transducer. The backscattered signal from an MES thus theoretically differs about a quarter in frequency, since the Doppler shift frequency depends on the insonation frequency (see appendix). Only a small number of MES originating from carotid artery plaques were analyzed, and moreover, the gold standard was only one human observer, which seems inappropriate especially in the case of low intensity MES. The results were not specified, i.e. it is not clear from their paper, what the contribution of the reference gate and the dual-frequency approach for the performance of their system was. Finally, is has to be kept in mind, that simultaneaus insonation with 2 different frequencies is likely to evolke different ultrasound fields and hence different backscatter content from the same embolus (Deverson et al. 2000).

As an alternative to evaluate MES within ultrasound signals, which have been processed extensively in conventional Doppler systems (see appendix), the so-called radiofrequency (RF) signal available at the front-end of the Doppler device can also be directly analyzed (Mess et al. 2002, chapter 5). This RF-based system provides a very hilgh true spatial resolution and furthermore is insensitive to possible signal saturation, which is a major problem for conventional systerms (e.g. Smith et al. 1994). Our data could not confirm the previously reported variability of MES characteristics, but instead we found a rather consistent pattern of MES signature, that even was comparable for gaseous and particulate MES. We, therefore, concluded that at least some of the MES characteristics 
described on the basis of standard Doppler processing are artefacts, mainly due to the large size of the sample volume of these TCD systems.

A possible example is the work of Devyust et al. (2000). Applying the matching pursuit (see above) they found that particulate MES had a more irregular (frequency) aspect than gaseous MES, i.e. the frequency distribution was more complex in particulate MES. Interestingly, these findings are in contrast to those of Smith et al. (1997), who found more frequency variability in gaseous MES. We could not see any frequency change, neither of gaseous nor of particulate MES, with the exception of gaseous MES showing a change of flow direction (Mess et al. 2002). So, it is likely that the changes described and analyzed in detail by Devyust et al. (2000) are the result of the interaction between the original embolic signal and the Doppler system used for acquisition and of further processing. Interestingly, a second study combining the matching pursuit with the dual gate technique (Devyust et al. 2001) could not replicate the findings of the data published earlier. It appears, that complicated signal analysis processing like the matching pursuit may be superfluous and even lead to erroneous results, and that a direct evaluation of the RF signal is more reliable. If the results of RF-based MES analysis are compared with the anatomy of the large intracranial arteries at the base of the skull, it appears, that with specific processing of the ultrasound RF signal it indeed is possible to track. MES with a high spatial accuracy (chapter 6). So, it is now evident, that MES characteristics as discussed earlier (Mess et al. 2002; chapter 5) were related to the actual anatomical situation. A disadvantage of our study, however, is the fact that two different ultrasound systems for the evaluation of the anatomy and MES detection are used, which unequivocally will introduce an error when comparing the data. So, for future research it will be preferable to perform MES detection with the same system being capable of visuallizing the MCA or other vessels of interest. In this way, the MES acquisition system could be adapted to the specific anatomical situation in a given patient more appropriately. 


\section{Future perspectives}

1. The role of signal analysis for defining MES: what is the "gold standard"?

The evaluation of automatic MES detection systems is unseparably related to the "human gold standard". If several human observers do not agree whether a given signal comprises an MES, the signal is not classified as an MES (Cullinane et all. 2000). MES of low intensity, which are more difficult to identify, often do not pass the judgement threshold of the expert panel. So, especially the detection of low intensity MES may suffer from an inferior gold standard. This might lead to a falsely too negative evaluation of an automated system. Low intensity MES, detected by the automated system, might be classified as non-MES by the human expert, which will result in a falsely low specificity of the automated system.

The use of a $1 \mathrm{MHZ}$ probe, which theoretically will increase the S/N of MES (Moehring and Klepper 1994 and Moehring and Ritcey 1996), in an in witro setting indeed produced relatively higher MES intensities compared to the usual $2 \mathrm{MHz}$ transducer (Cullinane and Markus 2001). This underlines the actual existance of low intensity MES which are discovered by the human gold standard, but that are undoubtedly present when a different technique is used. However, it only makes, sense to try to diagnose MES which are clinically relevant. Because an MES seldom will result in ischemia (Claus et all. 1999), but instead indicates the presence of an active embolic source, the question of the smallest, still clinically meaningful MES can hardly be answered.

Besides the intensity increase of an MES, there is, however, another sime qua non property of an MES, that discriminates it from the Doppler background and artefacts. As already the word "ermbolus" indicates (Stedman 1990), the particle giving rise to the MES, moves consistently through the blood and relative to the transducer. The Doppler speckle of the blood also exhibits small intensity fluctuations, which might be confused with low intensity MES. However, these intensity increases are likely to be of a shorter duration than those caused by MES. The dietection of a moving intensity increase could possibly perform as a better gold standard than the human observer expert panel.

In the future, the RF based amplitude plot could serve as a means for detecting such a movement. However, as was discussed in chapter five, a substantial portion of the particulate MES, which generally have a low intensity, could not be analyzed in terms of velocity and flow direction, presumably due to an inferior $S / N$. Since we applied a very short burst length, the emitted ultrasound power was comparably low. Theoretically, an increased amplitude of the emitted ultrasound bursts should result in a more reliable analysils of low intensity MES.

\section{Requirements for an automatic MES detection system}

It is obvious that automatic MES detection systems have to take into account that MES from different sources (i.e different intensities and different impedance) are likely to behave different 
with regard to the Doppler background signal. So, automated systems should have different "presets" for different clinical situations. Consequently, the performance of an automated MES detection system should be evaluated for the different saurces of MES before that system is. applied in a specific group of patients (Reihill et al. 2002). As was shown by v.Zuilen et al. (1996; chapter 2), an independent evaluation of an automated system has to reveal if a given system indeed offers ubiquitous applicability.

\section{Clinical use of MES detection}

Preliminary data suggest that in hitherto asymptomatic patients with a carotid artery plaque the detection of MES can discriminate between those that will lead to cerebral ischemia and those that will not (Siebler et al. 1995, Babikian et al. 1997, Valton et al. 1998). This information would be clinically important, since the degree of stenosis only weakly predicts a stroke or TIA. Plaque. characterization with $\mathrm{B}$-mode ultrasound has shown that specific properties are correlated with cerebrowascular events (European Carotid Plaque Study Group 1995), yet, this method untill now has not gained clinical relevance, which in part is due to the low interobserver agreement (Arnold et al. 1999, De Bray et al. 1998).

Possibly, MES detection will in the future help to identify high risk atherosclerotic carotid artery plaques. The relatively low incidence of MES in these patients, however, requires a large scale study to elucidate the predictive value of the prescence of MES (Mess and Hennerici, 2001).

So, theoretical $y$, in these patients an automatic detection system would be advantageous, but requires a very high specificity due to the large amount of negative Doppler signals, which is likely to result in inferior performance of automatic MES detection systems (Reihill et al 2002). Currentlly, the ACES study is underway, including 600 asymptomatic patients with a stenosis of more than $70 \%$ (Cullinane and Markus 2002).

To conclude, the work presented in this thesis reflects the complexity of MES description and hence detection. The interplay of the original ultrasound signal and its processing in a conventional Doppler system will lead to erroneous assumptions on MES characteristics. This process also hampers the dual gate technique, that aims at the moving nature of MES. However, if the radiofrequency sigmal directly is analyzed, the true MES signature becomes visible, revealing a surprisingly consistent pattern and clearly depicting the change of the intensity increase in time and place. 


\section{References}

Arnold JAC, Modaresi KB, Thomas N, Taylor PR, Padayachee TS. Carotid plaque characterization by duplex scanning; observer error may underrnine current clinical trials. Stroke 1999;30:61-65.

Aydin N, Padayachee S, Markus HS. The use of the wavellet transform to describe embolic signalls. Uitrasound Med Biol 1999;25:953-958.

Babikian VL, Wijman CAC, Hyde C, Cantelmo NL, Winter MR, Baker E, Pochay V. Cerebral microembolism and early recurrent cerebral or retinal ischemic events. Stroke 1997;28:1314-1318.

Brucher R, Russell D. Automatic embolus detection with artefact suppression. (Abstract) Neuroimaging 1993:3:77.

Brucher R, Russell D. Automatic online embolus detection and artifact rejection with the first multifrequency transcranial Doppler. Strake 2002;33:1969-1974.

Claus SP, Louwerse ES, Mauser HW, van der Mee M, Moll FL, Mess WH, Ackerstaff RGA. Temporary occlusion of middle cerebral artery by macroembolism in carotid surgery. Cerebrovasc Dis 1999;9:261-264.

Cullinane M, Reid G, Dittrich $R_{r}$ Kaposzta $Z_{r}$ Ackerstaff R, Babikian V, Droste DW, Grossett D, Siebler M, Valton $L_{s}$ Markus. HS. Evaluation of new online automated embolic signal detection algorithm, including comparison with panel of international experts. Stroke 2000;31:1335-1341.

Cullinane $\mathrm{M}_{*}$ Markus HS. Evaluation of a $1 \mathrm{MHZ}$ transducer for transcranial Doppler ultrasound including embolic signall detection. Ultrasound Med Biol 2001;27:795-800.

Cullinane M, Markus H. Asymptomatic carotid emboli study (ACES). (Abstract) Cerebrovasc Dis 2002;13(suppl 4):32.

de Bray JM, Baud JM, Delanoy P, Camuzat JP, Dehans V, Descamp-Le Chevoir J, Launay JR, Luizy F, Sentou Y, Cales P. Reproducibility in ultrasonic charactierization of carotid plaques. Cerebrowasc Dis 1998:8:273-277.

Deverson S, Evans: $\mathrm{DH}_{4}$ Bouch DC. The effects of temporal bone on transcranial Doppler ultrasound beam shape. Ultrasound Med Biol 2000;26:239-244. 
Devuyst $G$, Vesin JM, Despland PA, Bogousslavsky J. The matching pursiuti a new method of characterizing microembolic signals ? Ultrasound Med Biol 2000;26:1051-1056.

Devuyst G, Darbellay GA, Vesin JM, Kemeny V, Ritter M, Droste DW, Molina C, Serena J, Sztajzel R, Ruchat $P$, Lucchesi $C$, Dietler $G_{2}$ Ringelstein EB, Despland PA, Bogousslavsky J. Automatic classification of HITS into artifacts or solid or gaseous emboli by a wavelet representation combined with dual-gate TCD. Stroke 2001;32:2803-2809.

European Carotid Plaque Study Group. Carotid artery plaque composition - relationship to dinical presentation and ultrasound B-mode imaging. Eur J Vasc Endovasc Surg 1995:10:23-30.

Fan L, Evans DH. A real-time and fine resolution anallyser used to estimate the instantaneous energy distribution of Doppler signals. Ultrasound Med Biol 1994;20:445-454.

Fan L, Evans DH. Extracting instantaneous mean frequency information from Doppler signals using the Wigner distribution function. Ultrasound Med Biol 1994;20:429-443.

Fan L, Evans DH, Naylor AR. Automated embolus identification using a rule-based expert system. Ultrasound Med Biol 2001;27:1065-1077.

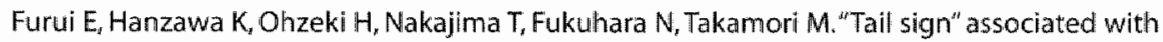
microembolic signals. Stroke 1999;30:863-866.

Georgiladis D, Kaps M, Siebler M, Hill M, König M, Berg J, Kahl M, Zunker P, Diehl B, Ringelstein EB. Variability of Doppler microembolic signal counts in patients with prosthetic cardiac valves. Stroke 1995;26:439-443.

Georgiadis D, Goeke J, Hill M, König M, Nabovi DG, Stögbauer F, Zunker P, Ringelstein EB. A novel technique for identification and Doppler microembollic signals based on the coincidence method; in vitro and in vivo evaluation. Stroke 1996;27:683-686.

Georgiadis D, Uhlimann F, Lindner A, Zierz S. Differentiation between true microembolic signals and artefacts using an arbitray sample volume. Ultrasound Med Biol 2000;26:493-496.

Kemeny $V_{*}$ Droste DW, Hermes 5 , Nabavi $D G$, Schulte-Altedorneburg $G_{*}$ Siebler $M$, Ringëlstein $E B$. Automatic embolus detection by a neural network. Strake 1999;30:807-810. 
Keunen RWM, Stam CJ, Tavy DLJ Mess WH, Titulaer BM, Ackerstaff RGA. Preliminary report of detecting microembolic signals in transcranial Doppler time series with nonlinear forecasting. Stroke 1998;29:1638-1643.

Kouamé D, Girault JM, Biard M, Patat F. Detection and characterization of embolic signals by parametric modeling. (Abstract) Cerebrovasc Dis 2002;13(suppl 4):33.

Lindner A, Georgiadis D, Fischer G, Zerkowski HR, Zierz S. Identification of Doppler microembolic signals with a bigate probe in patients with prosthetic heart valves. Eur J Med Res 1997;2:299-301.

Markus H, Loh A, Brown MM. Computerized detection of cerebral emboli and discrimination from artifict using Doppler ultrasound. Stroke 1993:24:1667-1672.

Markus HS, Reid G. Frequency filtering improves ultrasonic embolic signall detection. Ultrasound Med Biol 1999;25:857-860.

Mark us HS, Cullinane M, Reid G. Improwed automated detection of embolic signals using a novel frequency filtering approach. Stroke 1999;30:1610-1615.

Mess WH, Titulaer BM, Ackerstaff RGA. An in vivo model to detect microemboli with multidepth technique. Preliminary results. (Abstract) Cerebrovasc Dis 1996;6(suppl.3):60.

Mess WH, Titulaer BM, Ackerstaff RGA. Discrimination and characterization of emboli: old and new aspects. In: New Trends in Cerebral Hemodynamics and Neurosonology. (Eds: Klingelhöfer J. Bartels E, Ringelsteiln EB) Elsevier, Amsterdam 1997, 355-363.

Mess WH, Titulaer BM, Ackerstaff RGA. Middle cerebral artery anatomy and characteristics of embolic signals - a dual gate computer simulation study. Ultrasound Med Biol 1999:25:531-539.

Mess $\mathrm{WH}_{i}$ Titulaer BM, Ackerstaff RGA. A new algorithm for off-line automated emboli detection based on the pseudo-Wigner power distribution and the dual gate TCD technique.

Ultrasound Med Biol 2000;26:413-418.

Mess WH, Hennerici MG. High Intensity Transient Signals. In: Cerebrovascular Ultrasound; Theory, Practice and Future Developments. (Eds: Hennerici M, Meairs S) Cambridge University Piress. Cambridge 2001, 297-316. 
Mess WH, Willigers JM, Ledoux LAF, Ackerstaff RGA, Hoeks APG. Microembolic signal description: A reappraisal based on a customized digital postprocessing system.

Ultrasound Med Biol 2002:28:1415-1423.

Moehring MA, Klepper JR. Pulse Doppler ultrasound detection, characterization and size estimation of emboli in flowing blood. IEEE Trans Biomed Eng 1994;41:35-44.

Moehring MA, Ritcey JA. Sizing emboli in blood using pulse Doppler ultrasound-I: verification of the EBR model. IEEE Trans Biomed Eng 1996;43:572-5:80.

Munts $A G$, Mess WH, Bruggemans EF, Walda LA, Ackerstaff RGA. Feasibility and relability of on-line automated microemboli detection after carotid endarterectomy. A transcranial Doppler study. Eur J Vasc Endovasc Surg 2003;25:262-266.

Reihill S, Kaposzta Z, Cullinane M, Markus H5. On-line automated detection of cerebral embolic signals from a variety of embolic sources. (Abstract) Cerebrovasc Dis 2002;13(suppl 4):32.

Ries F, Tiemann K, Pohl C, Bauer C, Mundo M, Becher $H$. High-resolution emboli detection and differentiation by characteristic postembolic spectral patterns. Stroke 1998;29:668-672.

Roy E, Abraham P, Montresor S, Baudry M, Saumet. IL. The narrow band hypothesis: an interesting approach for high intensity transient signals (HITS) detection.

Ultrasound Med Biol 1998:24:375-382.

Siebler $M$, Rose $G$, Sitzer $M$, Bender $A$, Steinmetz $H$. Real-time identification of cerebral microemboli with US feature detection by a neural network. Radiology 1994;192:739-742.

Siebler $M$, Nachtmann A, Sitzer $M$, Rose $G_{r}$ Kleinschmidt A, Rademacher J, Steinmetz $H$. Cerebral microembolism and the risk of ischemia in asymptomatic high-grade internal carotid artery stenosis. Stroke 1995;26:2184-2186.

Smith $\mathbb{L}_{\mathrm{L}}$ Evans, DH, Fan $\mathrm{L}$, Thrush AJ, Naylor AR. Processing Doppler ultrasound signals from bloodborne emboli. Ultrasound Med Biol 1994:20:455-462.

Smith JLL, Evans. DH, Fan L, Gaunt ME, London NHM, Bell PRF, Naylor AR. Interpretation of embolic phenomena during carotid endarterectomy. Stroke 1995:26:2281-2284.

Smith $\mathbb{L}_{\mathrm{L}}$ Evans $\mathrm{DH}_{4}$ Fan L, Bell PRF, Naylor AR. Differentiation between emboll and artefacts using dual-glated transcranial Doppler ultrasaund. Ultrasound Med Biol 1996:22:1031-1036. 


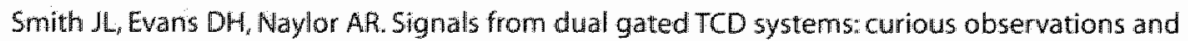
possible explanations. Uitrasound Med Biol 1997;23:15-24.

Smith $\mathrm{L}_{\mathrm{r}}$ Evans $\mathrm{DH}$, Naylor AR. Analysis of the frequency modulation present in Doppler ultrasound signals may allow differentiation between particulate and gaseous cerebral emboli.

Ultrasound Med Biol 1997;23:727-734.

Stedman TL. Stedman's medical dictionary. Baltimore Williams \& Wilkins, 1990.

Valton L, Larrue $V$, Pavy le Traon A, Massabuau P, Geraud G. Microembolic signals and risk of early recurrence in patients with stroke or transient ischemic attack. Stroke 1998;29:2125-2128.

van Dijk AD, de Wilde RBP, van der Rijken RAE, Bruggemans EF. Evaluation of automatic classification of HITS based on fundamental tone analysis. (Abstract) Cerebrovasc Dis 2002;13(suppl 4):32.

van Zuilden EV, Mess WH, Jansen C, van der Tweel I, van Gijn J, Ackerstaff RGA. Automatic embolus detection compared with human experts; Doppler ultrasound study. Stroke 1996;27:1840-1843. 
8

ADoenth

Sunimary

Samanawing

7usaminenfosung

Dankwoord

Divisalons.

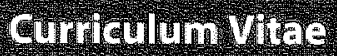





\section{Basic aspects of Doppler systems}

\section{Continuous wave Doppler systems}

The most simple Doppler system suited for measuring blood flow velocity in human beings is a continous wave (cw) Doppler device. The system has a master oscillator with a frequency usually between 2 and $8 \mathrm{MHz}$. Through a transmitting amplifier electrical energy is transferred to a socalled transducer, which converts the electrical into acoustical energy (piezo-electrical effect) by means of a transimitting crystal.

The emitted acoustical energy is concentrated within a narrow region, the ultrasound beam. If this beam is directed towards a medium, feflection and scattering will ocsur, depending on the wavelength (which is $0.77 \mathrm{~mm}$ in the case of $2 \mathrm{MHz}$ ) and on the transition in acoustic impedance (equall to mass density times sound propagation speed). Reflection occurs at rather large ( $>$ wavelengthy and smooth surfaces. If structures smaller than the wavelength are encountered, the ultrasound signal will be scattered in many directions, so that only a small part of the siginal will be returned or 'backscattered" to the transducer. The relation between the incident and backscattered ultrasound energy is called 'scattering cross section'.

Moving structures with a velocity w with respect to the transducer will induce a shift in frequency $f_{d}$ (Doppler shift frequency).

$\frac{f_{d}}{f_{a}}=\frac{2 v}{c}$

Where $f_{e}$ is the frequency of the incident signal and $c$ the velocity of ultrasound in the medium insonated. If the path of the scatterer has an angle $\alpha$ with respect to the ultrasound propagation direction, $v$ has to be replaced by $v \cos \alpha$. Doppler systems designed for measuring blood flow velocity use the backscatter signal caused by red blood cells (diameter $\sim 0.008 \mathrm{~mm}$ ) for further signal processing.

The sum of backscattered and reflected ultrasound is picked up by a second crystal and converted again into electrical energy, the radio-frequency (RF) signal. If the RF-signal includes backscatter from mowing blood, it will contain, due to the Doppler effect, signals with a different frequency than the reference signal. Mixing of the received signal with the reference signal results in signals in the audio range. This signal is made audible after low-pass filtering to remove all frequencies abowe the audio range and high-pass filtering to remove the strong reflections of stationary signals, the so-called "clutter" (typical value $100 \mathrm{~Hz}$ ). The process of extracting the audible Doppler signal from the RF-signall is called demodulation.

In simple cw-Doppler systems, the evaluation of the audible Doppler signal relies on the interpretation via a loudspeaker, while the average principal frequency of the Doppler signal is estimated by means of a zero-crossing method. More advanced systems allow for the 
differentiation between a Doppler shift towards and away from the probe and apply a spectrum analyzer. A continuous line on top of the spectirum ('envelope') permits more sophisticated analysis of blood flow velocity. Figure 1 shows a typical Doppler spectrum with an envelope.

Pulsed wave and transcranial Doppler systems

A pulsed wave (pw) Doppler system is a further refinement of the $\mathrm{c} w$ system described and permits to detect Doppler frequency shifts at a specific distance from the probe. Sa, a given blood flow velocity measurement can be assigned to a depth relative to the ultrasound transducer. In a pw Doppler system, the ultrasound is sent off intermittently, i.e. in the form of short pulses. For this purpose, a gate is connected between the master oscillator and the transmitting amplifier. This gate is controlled by a logic unit, which sets the length and the frequency of the emitted pulses, the latter being known as the pulse repetition frequency (PRF). In a typical commercial transcranial Doppler (TCD) system the ultrasound pulses have a length of $\sim 10 \mu$ s and the PRF may vary between approx. 4 and $16 \mathrm{kHz}$, depending on the depth of interest and the Nyquist theorem (PRF has to be at least twice than maximally measurable Doppler frequency shift).

The transducer in a pw Doppler system has only one crystal that alternately sends an ultrasound pulse and subsequently receives the backscattered signal from that pulse. Figure 2 illustrates the interaction between the ultrasound burst and the red blood cells.

The time interval elapsing between emission and the selection of the signal for further processing governs the depth (figure 3) assuming an ultrasound propagation speed of $\sim 1540 \mathrm{~m} / \mathrm{s}$. Commercial transcranial pw Doppler systems allow for depth adjustment in $\mathrm{mm}$, which makes it feasible to collect selectively information from the different major arteries at the base of the skull. In order to be able to penetrate the skull, most systems apply a relatively low insonation frequency on the order of $2 \mathrm{MHz}$.

The region, from which the backscattered signal is analyzed, is called the sample volume (SV). The size of the SV depends theoretically on several factors, with the length of the ultrasound burst and the time the receiver gate is open as the most important parameters. In general, the $5 V$ consists of a sensitive core, that is surrounded by decreasingly sensitive parts in the longitudinal as well as in the transversal axes. The lateral sensitivity decrease depends on the transducer characteristics. The recewer gate plays a crucial role for the sensitivity distribution along the longitudinal axis (depth), as is illustrated in figure 4.

The sensitivity also depends on the overall gaim of the system (Amolds et al. 1989), which implies that strong backscattering signals will be picked up over a larger SV than weak backscatterers. If transcranial Doppler (TCD) is considered, the architecture of the $5 V$ is even more complex, since the skull is likely to evoke distortions of the ultrasound beam (Deverson et al. 2000).

The information on blood flow velocity as analyzed with a pw Doppler system mostly is displayed in the frequency domain, using the fast Fourier transform (FFT). This form of spectral data presentation permits that the contribution of different frequencies (i.e. velocities) to the Doppler signal is made visible over time. Alternatively, the Doppler signal can be displayed in the time 


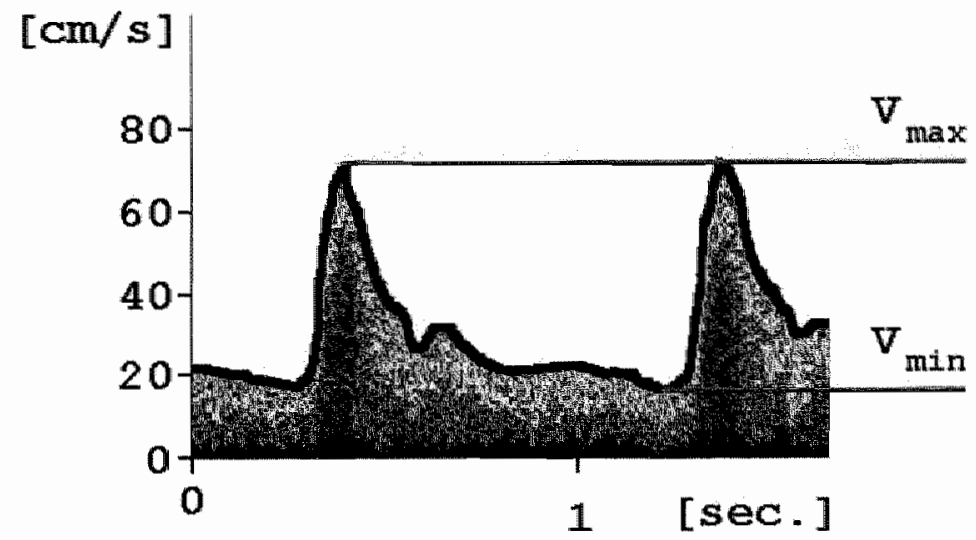

\section{Figure 1}

Fast Fourier Transform of a Doppler signal from the internal carotid antery. A byical fast Fourier transform (FFT) from a Doppler signal stemming from the internal carotid artery is shown with the Doppler shift (converted from frequency to welocity) on the $y$ axis (in $\mathrm{cm} / \mathrm{s}$ ) and the time on the $\mathrm{x}$-axis (in $\mathrm{sec}$. The presence of the different frequencies at a given time is color-coded whith the yellow and red tones indicating higher and the black and blue tones indicating lower values (green intermediate). The black line on top of the FFT spectrum is the so-called "einvelope",indicating the maxtmum velocity. The highest value / $v_{\text {inax }}$ " comresponds with the systole, the lowest value $f^{\prime}$, wit? " indicates the end of the diastole. The awerage of all values of the envellope during onte heart beat is

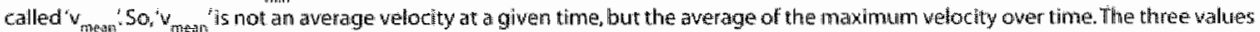

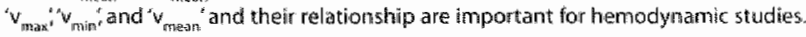

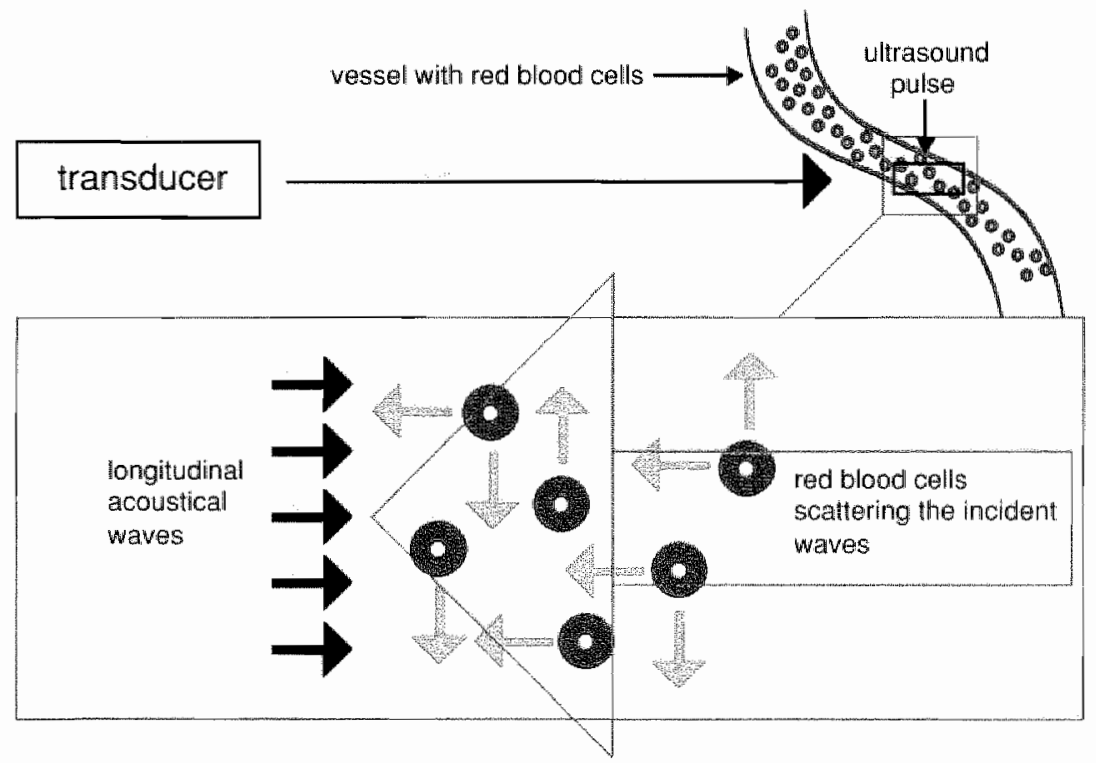

Figure 2

Figuire 2

Interaction between ultrasound bursts and moving blood. From the ultrasound transctucer a burst (black box of short lasting longltudinal waves has been ermitted, which encounters the red blood cells of a ves gel. Entargement: the witted ulbasound bifack arrows) is scattered by the erythrocytes in many directions (small gray arrows), howewer, as a net rescilt, a smallamount of uitrasound (liarge gray arrow) will be sent back to the tramsducer. The relation between the incident intensity (bidck arrows., and the power which is scattered baick, is called "scattering cross siection' Since the angle of insonation is decisive, the scattering cross section will be maximal at 180 degrees. This is known as the backscatter cross section: 
Figure 3 t1:

pulse is sent off
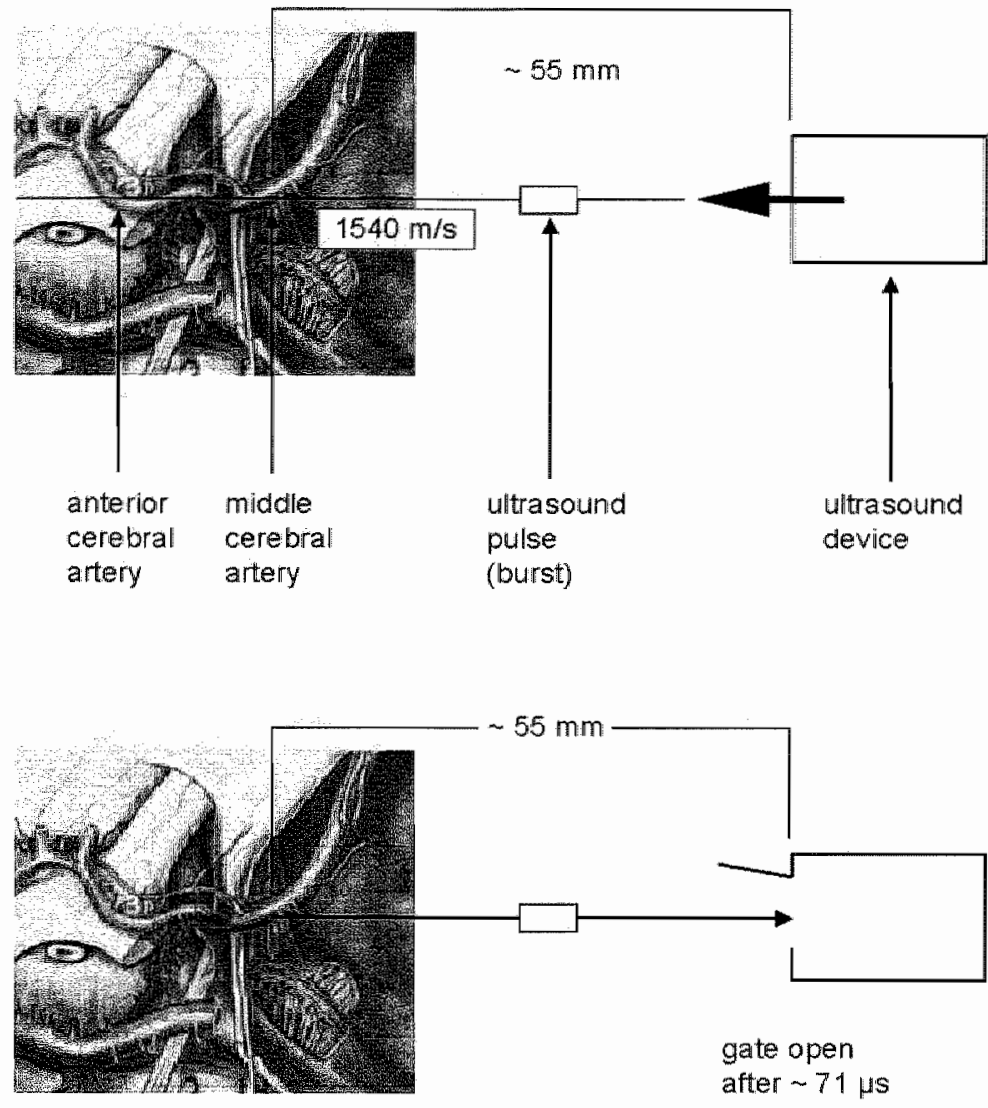

t2:

reflection of pulse is received after 71 HS; signal stems from MCA
13:

rellectian of pulse Is received after 87 Hs; signal stems from ACA

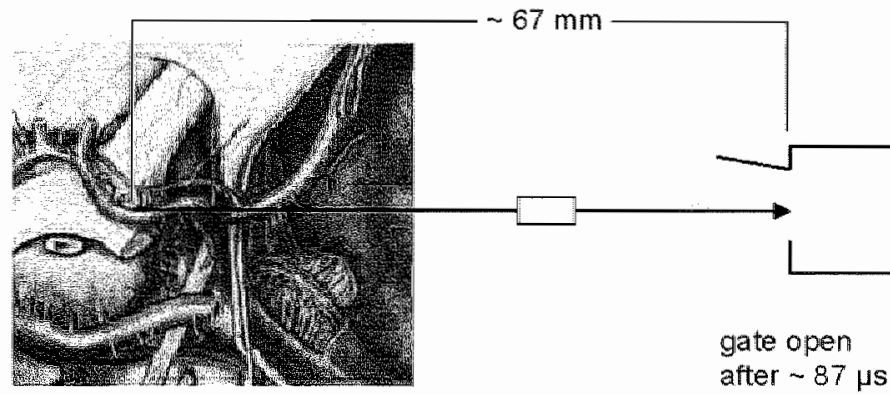

Figirare 3

Pulsed wave Doppler: Determination of depth. On the left side, the anatomical situation af the proxirnal parts of the middle and anterion cerebral artery is shown in a horizontal plane from above. On the right side, the whole pw Doppler system is drawn as a box, At 41 (top); an ultrasound pulse (burst") is emitted from the transducer. The welocity of this pulse is assumed to be $1540 \mathrm{~m} / \mathrm{s}$. On its whole trajectory, the ultrasound burst will evoke reflections and/or scattering. If the receiver gate is set open approximately 71 a s after the pulse had been sent it2; middle), only the information stemming from approximately 55 mm depth will be collected, which usually corresponds to a signal from the middle cerebral artery. If the receiver gate is set cpen approximately $87 \mu$ s after the pulse had been sent ( 3 ; bottom, the signal stemming from a depth af approximately 67 mm depth will be analyzed. This signal then is likely to stem from the anterior cerebral artery. 

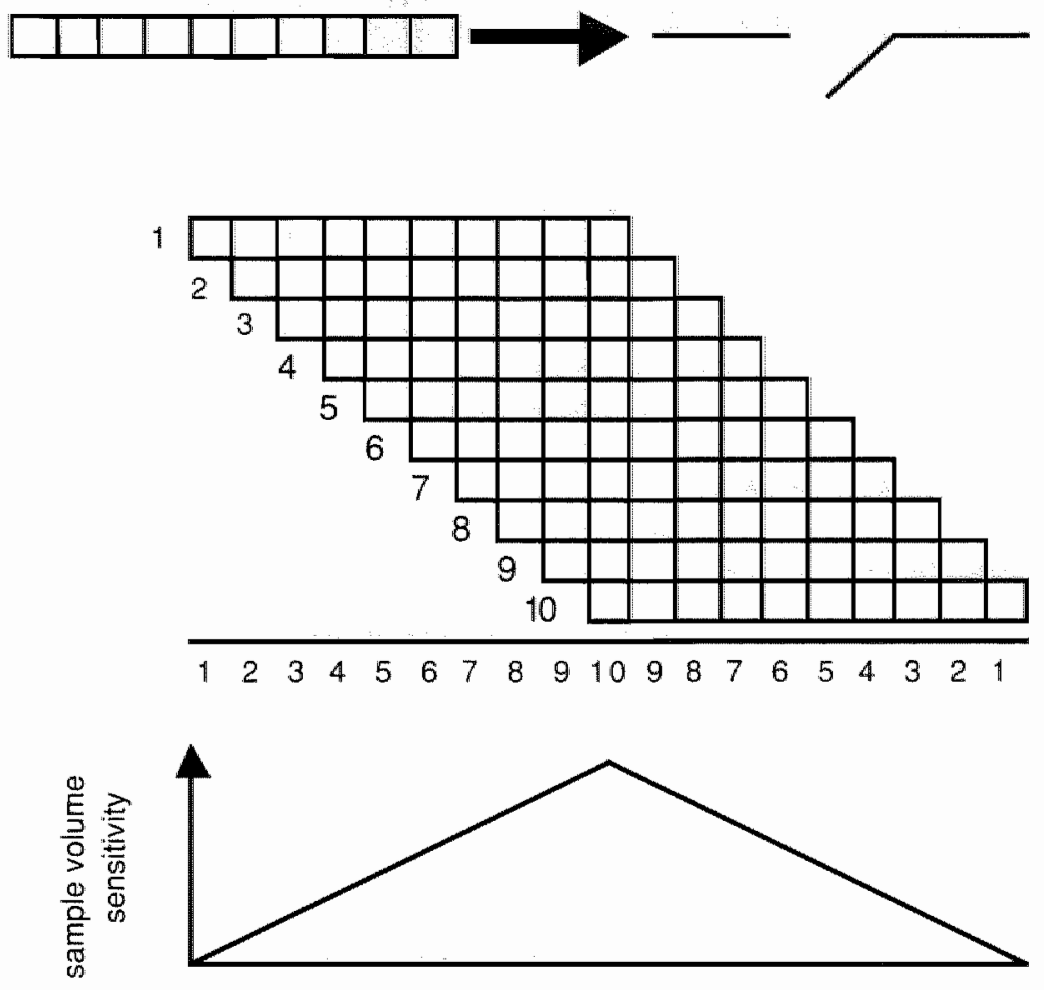

sample volume length $\sim 14 \mathrm{~mm}$ (19us)

\section{Figure 4}

The sample volume: burst length and receiver gate the. The figure explains how the receiver gate time contributes to the sensitivity distribution of the sample wolume (SW). An uttrasound bursti with a length of 10 flys is depicted and assumed to return to the transducer. The receiver gate time equals the ultrasound burst length $(10 \mu 5)$, a geting that is common in most commercial pwo Dopplen systems (top).

Schematically (middle), the incoming scattering and/or reflection from the ultrasound burst is analyzed 10 us long (figures 1 - 10 before ach row). However, in that time intervall the inconing signal does not st and still, so effectively the time analyzed is not: 10 us: (equaling $7.5 \mathrm{~mm}, 5 \mathrm{~V}$ ), but $19 \mu$ s (equaling $14 \mathrm{~mm} 5 \mathrm{~V}$; bottom). Furthernore, it is evident, that the sensit wity is not equally distributed within the SV (Peronneau at al. 1974). Instead, the senstivity has a triangle tike pattern (bottom) with the most sensitive part un the center and a part of increasing semsitivity at the begining and of decrousing serng itwity at the end.

domain, which is especially suitable for the analysis of microembolic signals (MES). Figure 5 shows an MES in both the frequency domain (FFT) and time domain.

The intensity of the backscattered signal from blood is not constant in time, but exhibits spontaneous, variations. These are caused by the accidental interaction of the incident ultrasound and the randomly distributed red blood cells. This phenomenon is called speckle and responsible for spontaneous intensity variations of up to $6 \mathrm{~dB}$ (Markus and Molloy 1997). Furthermore, the Doppler signal amplitude exhibits cyclic changes diuring one cardiac cycle (Paeng ell al, 2001). Both effects have to be taken into account if relative intensity increases of MES with respect to the Doppler background are calculated. 


\section{Figure $5 a$}

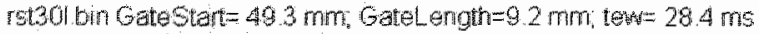

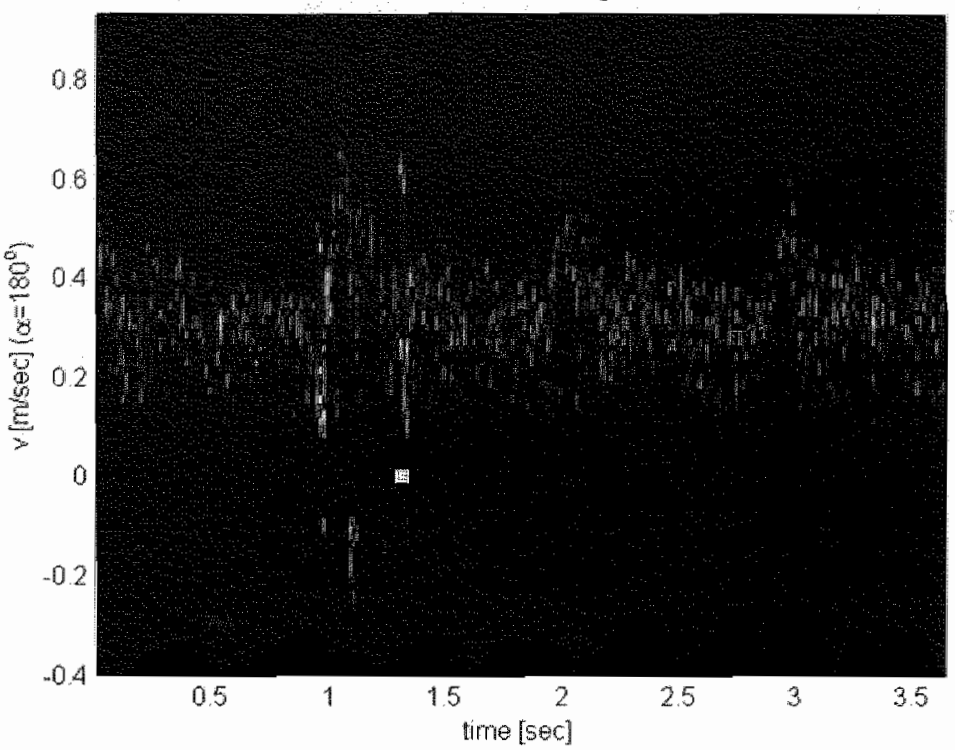

b

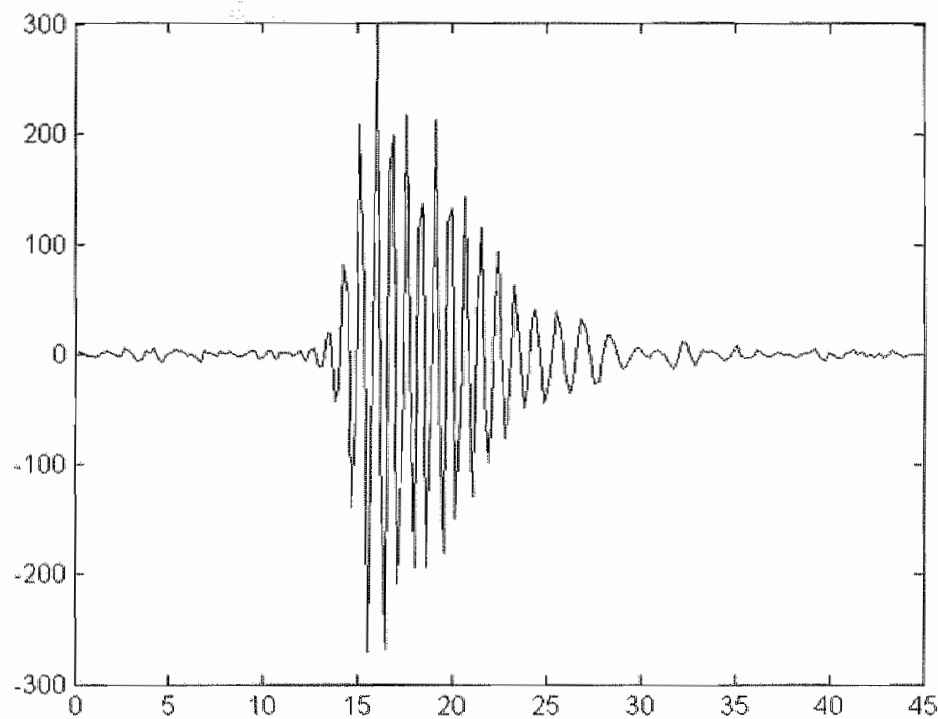

\section{Figure $5 \mathrm{a}$ and $\mathrm{b}$}

A mictoembolic signal in the frequency and time domain. a) The Doppler signal is dispiayed as an FFT amplitude spectrum with the intensity of the different parts coded in color isee figure 1]. However, the sen sitivity of the system was sel to very low values, so that the background folooe signal is displayed only in blue tones. The microembolic signals (MES) appear at approximately 1 and 1.3 seconds as very intense and short-lasting bars. The frequency is difficult to estimate, in the case of the second MES the high inten sity bar ewen exceeds the background signal. This would indicate an MES welocity higher thizan that from the s.urrounding blood. b) The Doppler signal of the time interval indicated by a yellow quadrangle in at is shown in the time domain with the intensity on the $y$-axis (arbitrary unts) and the time on the $x$-axis (sec). This technique dlearly allows for a better estimation of the temporal characteristics of the MES and shows the variabie frequency content with relatively low values at the end of the MES. Furthermore, calculations on the infensily of the MES compared to the Doppler signal from the blood signal without MES can be periormed. 


\section{References}

Arnolds $\mathrm{BJ}$, Kunz $\mathrm{D}_{\mathrm{z}}$ von Reutern GM. Spatial resolution of transcranial pullsed Doppler technique in vitro evaluation of the sensitivity distribution of the sample volume. Ultrasound Med Bial 1989:15:729-735.

Deverson S, Evans DH, Bouch DC. The effects of temporal bone on transcranial Doppler ultrasound beam shape. Ultrasound Med Biol 2000;26:239-244.

Evans $\mathrm{DH}_{,}$McDicken WN:Doppler ultrasound; physics, instrumentation and signal processing, 2nd ed. Chicester, Wiley \& Sons, 2000.

Markus HS, Molloy J. Use of a decibel threshold in detecting Doppller embolic signals.

Stroke 1997;28:692-695.

Paeng DG, Cao PJ, Shung KK. Doppler power variation from porcine blood under steady and pulsatile flow. Ultrasound Med Biol 2001;27:1245-1254.

Peronneau PA, Bournat JP, Bugnon A, Barbet A, Xhaard M. Theoretical and practical aspects of pulsed Doppler flowmetry: real-time application to the measure of instantaneous velocity profiles in vitro and in vivo. In Reneman RF (ed): Cardiovascular applications of ultrasound. Amsterdam, North Holland Publishing Group, 1974, pp. 66-84. 
Summary A stroke or cerebrovascular accident (CVA) is the third leading cause of death in most western countries and is responsible for decrease of quality of life in a substantial number of human beings. Mostly, the culprit is a lack of sufficient blood supply to parts of the brain due to a pathological proces in the arterial vessels. Roughly, two pathophysiologicall mechanisms can be identiffed. First, atherosclerosis can grow locally, typically in the carotid arteries in the neck and gradually reduce the patency. A stenosis or even occlusion develops, and consequently, less, blood will flow through that vessel to the brain.

Second, atherosclerosis can give rise to the formation of a thrombus, which can tear off and cause embolism distally. Besides these rather large emboli, so-called "microemboli" can emerge from an atherosclerotic plaque of e.g. the carotid artery. There is evidence that these microemboli are associated with an increased risk of cerebral infarction. Emboli appear in the Doppler audio signal as sudden and short lasting changes in amplitude. These are called high intensity transient signals (HITS) or "microembolic signals" (MES). This thesis focusses on the temporal and spatial properties of these MES as observed with transcranial Doppler (TCD) sonography in the middle cerebral artery (MCA).

\section{Chapter 7}

\section{Introduction}

First, on overview of the extracranial and transcranial blood vessels is given. Second, atherosclerosis and its relation to stroke are discussed. Atherosclerosis is regarded as a multifactorial disease, which starts early in life and comprises genetic, hemodynamic, environmental and infectious pathophysiological mechanisms. Typical preference sites of atherosclerotic plaque formation are arterial bifurcations. In terms of stroke, the branching off of the common carotid artery into the internal and external carotid artery is of paramount interest. If a plaque caused symptoms, it can be removed surgically or its thrombogenic effect treated with aspirine.

The surgical procedure ("carotid endarterectomy") can effectively be guarded by continuous noninvasive monitoring of the blood flow in the MCA by TCD. This technique is not only suited for measuring hemodynamic parameters, but also permits detection of microemboli because its passage is accompanied by an intensity increase in the Doppler audio signal, producing a characteristic sound. The microemboli can be either gaseous or particulate in nature. The former ones, are regarded as relatively benigin, the latter ones are potentially diangerous in terms of cerebral infarctions. During and after carotid endarterectomy microembolli detection can add significant information for patient care.

The main objectives of this thesis, as outlined in the introduction, are the evaluation of the interplay of micraembolic signal appearance and their detectability with special emphasis on the moving nature of microembolic signals. 


\section{Chapter 2}

The second chapter discusses three commercially available automatic embolus detection systems, which are based on the analysus of signals from one sample wolume. We compared these computer algorithms with four human observers. A total of 280 minutes of TCD signal from 10 patients with a caratid artery stenosis was analysed for the occurrence of MES. This resulted in a striking difference of the interobserver agreement between the manual approach, which was very high, and the automated approach, which was mediocre when compared with the human gold standard.

\section{Chapter 3}

A potential candidate for improvement of the automated approach is the so-called dual gate technique. Two sample volumes are placed sequentially in the MCA, which theoretically should result in time lag when a microembolus passes. An artefact on the other hand should appear simultaneously, thus allowing a high accuracy for a valid microembolus detection. In a preliminary study we found a remarkable variation of the time lag values and concluded that this variation depends on the acoustic properties of the embolus, the TCD settings, and the anatomy of the MCA. We, therefore, developed a computer simulation, that allowed to adjust all crucial TCD settings ais well as the anatomy of the MCA. Chapter three focuses on the effect of different TCD settings and anatomical situations of the MCA, as observed with the computer model. It could be shown that a proper consideration of the anatomy of the MCA is crucial for a successful dual gate approach. If the gate separation is as large as $10 \mathrm{~mm}$, the curved course in space and the variation of the MCA mainstem length are likely to contribute to a marked variation of the time lag values, which renders the dual gate technique questionable as a trustworthy instrument for an automated emboli detection system. However, when applying a smaller gate separation ( $4 \mathrm{~mm}$ ), this variation could be limitted substantially.

\section{Chapter 4}

In the fourth chapter a newly developed algorithm is presented, based on the dual gate technique and the pseudo Wigner power distribution. The latter was chosen, because of its superiority compared to the Fourier transformation in terms of a high resolution in both the temporal and the frequency domain. We manually selected MES and artefact signals from 20 surgical procedures and tested the algorithm for its capability to correctly identify the signals. The accuracy of the algorithm nearly reached the level of the human interobserver agreement, however, only if the MES were present in both sample volumes (which was the case in approximately $75 \%$ of the signalls). Additionally, electrocautery was insufficiently rejected as an artefact.

\section{Chapter 5}

The different signature of an MES in two serially placed sample volumes still proved to be a major shortcoming of automatic MES detection. Therefore, a radiofrequency (RF) based system for MES description was developed. Basically, a conventional Doppler system was modified, so that the 
received RF signal, which contains all information from the Doppler probe before any further processing was made externally available. This signal together with the internal clock of the Doppler machine and the emission trigger, which determines, when an ultrasound burst is sent off by the probe, were fed into an external computer. This RF based analysis system had a spatiall resolution of about 2 mim and allowed for plotting the RF amplitude as a function of time and depth. The appearence of MES was rather consistent in terms of depth range and velocity variation. It could indeed be shown that the rather large siample volumes of conventional Doppler systems are responsible for the remarkable variability of MES appearence.

\section{Chapter 6}

Applying the RF based system, we observed in about 30\% of the gaseous MES at specific depths a change of flow direction. Chapter six presents the study comparing these depths values with the information of the vessel anatomy as seen with transcranial color coded duplex sonography. On the day before open heart surgery, we documented in five patients the depth of the bifurcation of the internal carotid artery into the middle cerebral artery and the anterior cerebral artery as well as any visible branches of the MCA mainstem. During surgery MES were sampled and the depth values of a flow direction change of the MES were estimated and compared to the individual anatomy as seen with transcranial color coded duplex sonography. It could be shown, that the depth values of both methods agreed with an accurracy of $2 \mathrm{~mm}$, demonstrating, that the RF based system is capable of tracing MES with a high spatial resolution.

\section{Chapter 7}

Epilogue

Chapter seven discusses the coherence of the results of all studies. Additionally, the perspective of recent developments in the field of automatic microemboli detection is discussed in general. First, different approaches based on a single sample volume in terms of signal analysis have been proposed, comprising a frequency filtering technique and a sophisticated "rulle-based expert system" which incorporates neural network aspects. Second, modifications of the dual-gate approach have been presented, including a combination with the wavelet technique and the application of a so-called arbitrary sample volume, which intentionally is placed outside a blood vessel and provides a reference background signal. Also, a dual-frequency technique has been introduced, making use of the specific backscatter properties of a microembolus with regard to the incident ultrasound frequency.

Finally, possible future perspectives are outined. The so-called "human gold standard" fial microembolus detection can be questioned, especially for low intensity signals. Possibly, the radiofrequency technique will be able to undoubtedly show the mowing nature of a microembolus, which will distinguish it from any other signall. It is obvious, that an automatic microembolus detection system should be tuned for specific sources of embolic material and that the performance of a given system should be tested independently. Besides the meanwhile proven use 
of microembolus detection in conjunction with carotid endarterectomy, the identification of potentially hazardeous carotid artery plaques will be a challenging field of research in the future.

\section{Chapter 8}

\section{Appendix}

In the appendix basic aspects of Doppler systems are explained. First, the principles of a continuous wave Doppler systems are outlined, followed by a general description of pulsed wave and transcranial Doppler systems. Special emphasis is paid to the spatial properties of the sample volume.

\section{Conclusion}

To conclude, the work presented in this thesis reflects the complexity of MES description and hence detection. The interplay of the original ultrasound signal and its processing in a conventional Doppler system will lead to erroneous assumptions on MES characteristics. This process also hampers the dual gate technique, that aims at the moving nature of MES. However, if the radiofrequency signal directly is analyzed, the true MES signature becomes visible, revealing a surprisingly consistent pattern and clearly depicting the change of the intensity increase in time and place. 
Samenvatting Een beroerte of cerebro-vascullair accident (CVA) is de op twee na meest belangrijke oorzaak voor overhijden in de meeste westerse landen. Daarnaast verlaagt een CVA de kwaliteit wan het leven. In de meeste gevallen kan een tekort aan bloed in delen van de hersenen op basis van pathologische weranderingen in de arteriën als boosidoener geïdentificeerd worden. In grote lijnen betreft het hilerbij twee pathologische processen. Ten eerste kan atherosclerose, oftwel aderverkalking, locaal groeien en geleidelfjk aan de bloeddoorstroming belemmeren. Een typische locatie van deze z.g. plaque is de hallsslagader (arteria carotis). Als gevolg hiervan ontstaat een vernauwing (stenose) of zelfs een volledige afsluiting (occlusie), waardoor er minder bloed naar de hersenen kan stromen. Ten tweede kan atherosclerose aanleiding geven tot het ontstaan van een thrombus, die losgerukt kain worden en verder in het vaatbed tot een occlusie in een bloedvat met een kleinere diameter kan leiden. Dit fenomeen heet embolisatie. Naast deze relatief grote embolieën komen ook z.g. "microembolieën" voor. Er zijn aanwijzingen dat deze microembolieën geassocieerd zijn met een verhoogd rísico op een CVA. De microembolieën wekken in het Doppler audio signaal een plotselinge en kortdurende amplitudeverhoging op. Deze worden "high intensity transient signals" oftewel "microembolic signals" (MES) genoemd. Dit proefschrift behandelt de temporele en spatiële eigenschappen van deze MES, zoals die met de transcraniële Dopplersonografie (TCD) in de arteria cerebri media, een belangrijke slagader in de hersenen, worden waargenomen.

\section{Hoofdstuk 1}

Introductie

Er wordt eerst een overzicht van de extra- en transcraniële bloedvaten gegeven. Daarna wordt atherosclerose, en in het bijzonder de relatie met CVA, besproken. Atherosclerose wordt beschouwd als een al op jonge leeftijd beginnende multifactoriële aandoening. De pathofysiologie omvat genetische, haemodynamische, unfectieuse en ook milieu aspecten. De splitsingen, oftwel bifurcaties, van arteriën zijn woorkeur locaties voor het ontstaan van atherosclerose. In het geval van CVA speelt de carotis bifurcatie, dl.w.Z. de splitsing van de arteria carotis communis in de a.carotis interna en externa ongeveer th. $v$. het midden van de hals, een crucialle rol. Indien een plaque symptomen veroorzaakt, dan kan die of chirurgisch verwijderd worden of het z.g. thrombotische potentiaal eirvan, $d . w$.z. de neiging om een thrombus en mogelijk embolieën te vormen, worden behandeld met b.w. aspirine.

Continue en noninvasief monitoring d.Im.v. TCD maakt een goede bewaking van de cerebrale bloedvoorziening tijdens de operatie (carotis desobstructie) mogelijk. Deze techniek leent zich niet alleen om de haemodynamische veranderingen b.y. bij het afklemmen van de arteria carotis te meten, maar men kan er ook micorembolieën mee detecteren, omdat de intensiteitswerhoging in thet Doppler audio signaal gepaard gaat met een karakteristiek geluid. De microembolieën bestaan voornamelijk uit lucht of uit vast materiaal. De lucht microembolieën worden als relatief ongevaarlijk beschouwd, de vaste microembolieën daarentegen kunnen cerebrale infarcten veroorzaken. 
Tijdens, maar ook na een carotis desobstructle kan het detecteren van mictoembolieen belangrijke informatie voor de behandeling van de patient leveren.

De voornaamste doelstelling van dit proefschrift is te onderzoeken hoe de detecteerbaarheid van microembolieën afhangt van de meettechniek en het bewegingsgediag van de embolieën.

\section{Hoofdstuk 2}

In het tweede hoofdstuk woirden drie commercieel verknijgbare automatische embolus detectie systemen besproken. Deze zijn allemaal gebaseerd op de analyse van signalen afkomstig uit één saimple volume. Wij hebben de drie computer algoritmen wergeleken met vier menselijke observers. In totaal 280 minuten TCD signaal van 10 patienten met een vernauwing van de arteria carotis interna werden geanalyseerd m.b.t. het voórkomen van MES. De overeenstemnning tussen de menselijke observers onderlijk was zeer hoog. De overeenstemming tussen de automatische systemen en de z.g. menselijke gouden standaard was daarentegen middelmatig.

\section{Hoofdstuk 3}

Een mogelijke kandidaat voor verbetering van de automatische MES detectie is de z.g."dual-gate techniek". Twee sample volumes worden achter elkaar in dle arteria cerebri media geplaatst. Dit: resulteert theoretisch in een tijdswerschil indien een microembolus eerst het proximale en vervolgens het distale sample volume parseert. Een artefact daarentegen zou tegelijkertijd in beide sample volumes moeten worden waargenomen. Dit principe zou met een hoge nawwkeurigheid een valide microembolus detectie mogelijk moeten maken, In een eerste studie vonden wij een zeer opvallende varuatie van de tijdsverschillen. Wij concludeerden dat deze wariatie afhankelijk is. van de acoustische eigenschappen van de embolus, de instelligen van de TCD apparatuur en de anatomie van de arteria cerebri media.

Wij ontwikkelden daarom een computer simulatie model. Daarmee was het mogelijk alle belangrijke TCD instellingen maar ook zeer uiteenlopende anatomische variaties van de arteria cerebri media aan te passen. In dit hoofdstuk worden de effecten van verschillende TCD instellingen en anatomische situaties wan de arteria cerebri media op MES beschreven zoals die met het computer model voorspeld werden. Wij zagen dat het rekening houden met de anatomie van de arteria cerebri media inderdaad cruciaal is voor het succesvol toepassen van de dual-gate techniek. Indien de sample volumes $10 \mathrm{~mm}$ uit elkaar liggen, zullen het bochtig weploop van de arteria cerebri media in de ruimte en de variatie in de lengte tot een substantiëte variatie van de tijdsverschillen leiden. Dit zet vraagtekens bij de dual-gate techniek als een betrouwbaar instrument voor de automatische detectie van microembolieën. Door de sample volumes dichter bij elkaar te plaatsen ( $4 \mathrm{~mm}$ afstand) kon de variatie echter in belangrijke mater beperkt worden.

\section{Hoofdstuk 4}

In dit hoofdstuk wordt een nieuw algoritme gepresenteerd, waarwan de ontwikkeling gebaseerd is op de dual-gate techniek en de pseudo Wigner power distribution. Deze werd gekozen wanwege 
de betere temporelie en spatiele resolutie vergeleken met de gebruikelijke Fourier transformatie. Er werden in eerste instantie MES and artefacten afkomstig van 20 chirurgische ingrepen geselecteerd. Vervolgens werd het vermogen wan het algoritme getest om deze signalen correct te identificeren. De nauwkeurigheid van het algoritme bereikte nagenaeg het niveau van de menselijke observers, echter alleen indien de MES in beide sample wolumes aanwijsbaar was. Dit was in ca. $25 \%$ van de MES niet het geval. Daarnaast bleek dat diatermie artefacten introduceerde, die niet altijd als zodanig herkend werden.

\section{Hoofdstuk 5}

De verschillende verschijningsvormen van een MES in twee achter elkaar geplaatste sample volumes bleken een belangrijk probleem van automatische MES detectie systemen op basis van de dualgate techniek te zijn. Er werd daarom een systeem voor de beschrijving van MES ontwikkeld dat op de direkte analyse van het radiofrequente signaal (RF signaal) van de TCD gebaseerd is. Een conventioneel gepulst Doppler systeem werd dusdanig gemodificeerd dat het RF signaal , dat alle oorspronkelijke informatie van de Doppler transducer bevat, exterm beschikbaar kwam. Dit signaal werd samen met de interne clock van het Doppler toestel en de z.g. emission trigger in een externe computer gesampled. De emission trigger geeft daarbij aan wanneer een ultrageluidspuls de transducer verlaat. De amplitudo van het antwangen RF signaal werd als functie van de tijd en de diepte grafisch getoond. Dit RF gebaseerde analyse systeem had een spatiële resolutie van ca. 2 mm. Het verschijnen van MES was vrij consistent met betrekking tot de diepte en de variatie van de snelheid. Wij concludeerden dat de grote variabiliteit van de $M E S$, zoals gezien met conventionele Doppler systemen, een artefact is en vooral berust op de zeer grote sample volumes.

\section{Hoofdstuk 6}

De resultaten van de RF anallyse lieten zien, diat ca. 30\% van de lucht MES op bepaalde dieptes, een verandering in richting ten opzichte van de ultrageluidstransducent toonden. Wij vergeleken vervolgens deze dieptes met de anatomie van de grote intracraniële bloedvaten, zoals d.m.v. transcraniële kleur gecodeerde duplex sonografie kan worden vastgesteld. Één dag voordat er een chirurgische ingreep aan het open hart plaats vond, werd bij viff patienten de diepte van de intracranièle afsplitsing van de arteria cerebri anterior uit de caratis interna gedocumenteerd, alsmede de diepte van afsplitsingen van bloedvaten uit de arteria cerebri media voor zover zichtbaar. Op basis van de anatomie kan ter plaatse een verandering van stroomrichting ten opzichte wan de ultrageluidstransducent verwacht worden. Tijdens de operatie werden de MES geregistreerd en vervolgens de diepte van een eventuele verandering van de stroomrichting vergeleken met de diepte wan intracraniële vaatafsplitsingen. Wij konden aantonen dat de dieptes van beide methoden met een hoge nauwkeurigheid van $2 \mathrm{~mm}$ overeenstemden. Dit demonstreert dat het RF gebaseerde systeem in staat is MES met een hoge spatiêle resolutie te traceren. 


\section{Hoofdstuk 7}

\section{Epiloog}

De resultaten van alle studies van dit proefschrift worden hier samenhangend besproken.

Daarnaast worden de actuele ontwikkelingen op het gebied van automatische microembolie detectie aan de orde gesteld.

Er ziljin verschillende manieren van signaal analyse uitgaande van één sample volume voorgesteld, onder andere een frequentie filter techniek en eem complex "rule-based expert system, dat aspecten van neuronale netwerken bevat. Daarnaast zijn modificaties van de dual-gate techniek gepresenteerd, onder andere in combinatie met de wavelet techniek en het z.g. "arbitraire sample volume." Dit sample volume wordt als referentie met opzet buiten het bloedvat geplaatst. Ook is er inmiddels een techniek, die op de analyse van twee gleluids swrequenties gebaseerd is. Deze aanpak maakt gebruik wan het feit dat een microembolus specifieke ultrageluidseigenschappen ten opzichte van de geluids-frequentie heeft.

Tenslatte worden mogelijke toekomstige ontwikkelingen geschetst. Er kunnen vraagtekens geplaatst worden bij de z.g."menselijke gouden standaard" vooral voor signalen met lage intensiteit. Met de RF techniek is het mogelijk de beweging van de microembolus eenduidig aan te tonen. Deze eigenschap onderscheidt de MES van alle overige signalen. Het is duidelijk dat automatische systemen voor microembolie detectie afgestemd moeten worden op specifieke bronnen van microembolieën. De prestaties van deze systemen moeten getest worden door onafhankelijke onderzoekers. Klinisch is gebleken dat het detecteren van microembolieëin zinvol is. De identificatie van potentieel gevaarlijke plaques im de arteria carotis interna d.m.v. emboliedetectie is een bellangrijke uitdaging voor de toekomst.

\section{Hoofdstuk 8}

\section{Appendix}

In de appendix wonden basalle aspecten wan Doppler systemen uitgelegd. Eerstw worden de principes van continuous wave Dopplier systemen beschreven, daalna volgt een algemene beschrijving van gepulste en transcraniële Doppler systemen. Er wordt vooral ingegaan op de spatiële eigenschappen van het sample volume (meetvolume).

\section{Conclusie}

Resumerend spiegelt dit proefschrift de complexiteit wan de MES beschrijving en dus ook detectie weer. Het samenspel van het oorspronkelijk ultrageluidssignaal en de verdere signaalwerwerking zal in conventionele Doppler systemen tot foute veronderstellingen m.b.t. de MES eigenschappen leiden. Dit fenomeen belemmert ook de dual-gate techniek, die zich richt op de aan MES inherente beweging. Indien echter het RF signaal zelf geanalyseerd wordt, kan de werkelijke verschijningsvorm van de microembolus zichtbaar worden gemaakt. Dit resulteert iri een verrassend consistent patroon, waarbij de veranderingen in intensiteit in plats en tijd duidelijk aangegeven kunnen worden. 
ZUSammenfassung schlaganfälle sind die dritthäufigste Todesursache in den meisten westlichen Ländern und verantwortlich für eine Einschränkung der Lebensqualtität vieler Patienten. Eine unzureichende zerebrale Blutzufuhr aufgrund krankhafter Veränderungen der Arterien spielt ursächlich die grösste Rolle. Im wesentlichen können zwei pathophysiologische Mechanismen unterschieden werden. Einerseits kann Atherasklerose lokal in der Halsschlagader (Arteria carotis) zu einer zunehmenden Verengung des. Lumens führen. Dadurch entsteht eine Stenose oder sogar ein Verschluss, was zur Folge hat, dass weniger Blut durch dieses Gefäss, zum Gehirn fliessen kann. Andererseits kann Atherosklerose zur Bildung eines Thrombus führen, der sich lösen und so eine Embolie im distalen Gefässbett verursachen kann. Ausser diesen relativ grossen Emboli können auch sogenannte Mikroemboli auf der Basis eines Plaques in der Arteria carotis entstehen. Es gibt deutliche Hinweise darauf, dass diese Mikroemboll ein erhöhtes Schlaganfallrisiko anzeigen. Im Doppleraudiosignal werden Mikroemboli als plötzlich auftretende Amplitudenveränderungen von kurzer Dauer wahrgenommen. Diese werden "high intensity transient signals (HITS)" oder auch "microembolic signals (MES)" genannt. Diese Promotion richtet sich auf die Analyse von Mikroemboli, die mit Hilfe der transkraniellen Dopplersonographie (TCD) in der Arteria cerebri media (ACM) beobachtet werden können. Dabei werden die Eigenschaften dieser Signale sowohl in der zeitlichen als auch in der räumlichen Dimension besprochen.

\section{Kapitel 1}

\section{Einleitung}

Zunächst werden die wichtigsten extra- und intrakraniellen Blutgefässe beschrieben, danach werden die Atherosklerose und ihre Beziehung zum Schlaganfall besprochen. Atherosklerose ist eine multifaktorielle Erkrankung, die bereits in jungem Alter beginnt und unter anderem auf genetischen, hämodynamischen und infektiösen Mechanismen beruht. Daneben können Umwelteinflüsse ebenfalls eine Rolle spielen. Sogenannte atherosklerotische Plaques sind bevorzugt an Gabelungen von Gefässen lokalisiert, auf Schlaganfälle bezogen, ist hierbei die Aufzweigung der Arteria carotis communis in die Arteria carotis interna und Arteria carotis externa von grossem Interesse. Wenm ein solelher Plaque klinische Symptome verursacht hat, dann kann dieser chirurgisch entfernt werden oder aber das thrombogene Potential, also auch die Fähigkeit, Ennboli zu generieren, kann mit Aspirin oder anderen Thrombozytenaggregationshemmern behandelt werden.

Eirn Monitoring des Blutflusses in der ACM kann während dieses chirurgischen Eingriffes ("Karotisentarteriektomie") kontinuierlich und nicht invasiv mittels TCD erfolgen. Diese Technik eignet sich aber nicht nur für die Messung der hämodynamischen Parameter, sondern gestattet auch die Wahrnehmung von Mikroemboli, da diese eine charakteristische Intensitätserhöhung im hörbaren Dopplersignal verursachen, welche wiederum von einem typischen Klang begleitet wird. Mikroemboli können gasförmiger Natur sein oder aber aus festem Material bestehen, unter anderem kleinen Thrombi. Gasförmige Mikroemboli werden im allgemeinen als relativ harmlos angesehen, wohingegen die zweite Gruppe von Mikroemboli auf eine potentielle Gefahr im 
Hinblick auf zerebrale Infarkte weisen kann. So kann das Auftreten von Mikroemboli wälurend und nach Karotisendarteriektomie wichtige Informationen und Entscheidungshilfen für die optimale Behandlung des Patienten liefern, da diese Mikroemboli sehr wahrscheinlich aus festem Material bestehen.

Die Hauptragestellung dieser Promotion ist die Evaluation des Zusammenhanges zwischen der Detektierbarkeit eines Mikraembolus, also der Möglichkeit, ihn vom übrigen Dopplersignal unterscheiden zu könmen, und der angewendeten Messtechnik einerseits sowie andererseits des Bewegungsverhaltens des Mikroembolus selbst.

\section{Kapitel 2}

Im zweiten Kapitel werden drei automatische Embolusdetektionssysteme besprochen, die auf kommerzieller Basis zur Verfügung stehen und auf der Signalanalyse aus einem Messvolumen beruhen. Wir verglichen diese Computeralgorithmen mit vier menschlichen Beobachtern, wobei deren Urteil als sogenannter "Goldstandard" angesehen wird. Insgesamt 280 Minuten TCD Signal von zehn Patienten, die eine Stenose der Halsschlagader hatten, wurden im Hinblick auf das Auftreten von MES analysiert. Dies resultierte in einem auffallenden Unterschied der sogenannten Interobserver Übereinstimmung: Diese erreichte sehr hohe Werte für die menschlichem Beobachter, wohingegen die Übereinstimmung zwischen den automatischen Systemen und dem Goldstandard im besten Falle mittelmässig war.

\section{Kapitel 3}

Eine Verbesserung der automatischen Analyse könnte möglicherweise durch die Anwendung der sogenannten "dual-gate" Technik erreicht werden. Hierbei werden zwei Messvolumina hintereinander in der ACM plaziert, was theoretisch in einer Zeitdifferenz resultiert, wenn ein Mikroembolus die Messvolumina passiert, ein Artefakt hingegen müsste gleichzeitig wahrgenommen werden. Diese Methode bietet also theoretisch die Möglichkeit einer genauen und validen Mikroembolusdetektion. In einer ersten Untersuchung fanden wir jedoch eine bemerkenswerte Variabilität der Zeitdifferenzen und folgerten daraus, dass diese Variabilität abhängig ist von den akustischen Eigenschaften des Embolus, den Einstellungen des TCD Gerătes und der Anatomie der ACM.

Wir entwickelten daher ein Computermodell, das uns erlaubte, sowohl alle wesentlichen TCD Einstellungen als auch verschiedene anatomische Situationen der ACM zu simulieren. In Kapitel drei werden die Effekte der verschiedener TCD Parameter als auch Gefässanatomien auf Mikroembolisignale beschrieben. Es konnte gezeigt werden, dass eine sorgfältige Berücksichtigung der anatomischen Gegebenheiten von essentieller Bedeutung für eine sinnvolle Anwendung der dual-gate Technik ist. Wenn der Abstand zwischen den beiden Messwolumina $10 \mathrm{~mm}$ beträgt, dann werden der kurvige Verlauf und die interindividuelle Wariabilität der ACM Anatomie wesentllich zu unverhersehbaren Werten für die Zeitdifferenzmessung führen, woclurch die dual-gate Technik fragwürdig wird als zuverlässiges instrument für die automatische Embolidetektion. Wir konnten 
Jedoch zeigen, dass die Variabilität der Zeitdifferenzen deutlich reduziert werden kann, wenn der Abstand zwischen den Messvolumina auf $4 \mathrm{~mm}$ reduziert wird und darüberhinaus die Messvolumeneigenschaften angepasst werden.

\section{Kapitel 4}

Wir entwickelten daraufhim einen neuen Algorithmus, der zum einen auf der duall-gate Technik basiert und zum anderen auf der sogenannten "Pseudo Wigner Power Distribution," die aufgrund der relativi besseren zeitlichen und räumlichen Auflösung verglichen mit der Fourier Transformation zur Anwendung kam. Es wurden zunăchst manuell kurze TCD Segmente ausgewählt, die während 20 chirurgischer Eingriffe aufgenommen worden waren und entweder MES oder Artefakte beinhaiteten. Im Anschluss daran untersuchten wir, inwiefern der neue Algorithmus in der Lage war. die Signale korrekt zu identifizieren. Es zeigte sich, dass der Anteil der richtig beurteilten Signale mahezu auf dem Niveau des mensclhlichen Goldstandard war, jedoch nur, wenn das MES im beiden Messvolumina vorlag, was nur in ca. $75 \%$ der Signale der Fall war. Darüberhinaus sahen wir, dass Elektrodiathermie als elektrische Störquelle nur in ungenügender Weise als Artefakt erkannt wurde.

\section{Kapitel 5}

Die Tatsache, dass ein Mikroembolus in zwei nacheinander angeordneten Messvolumina unterschiedlich wahrgenommen wird, stellt den grössten Mangel der automatischen Embolidetektion auf Basis der dual-gate Technik dar. Wir haben darum ein System für die Detektion won MES auf der Grundlage des sogenannten Radiofrequenzsignal (RF-signal) entwickelt. Dafür wurde ein konventionelles Dopplersystem dahingehend madifiziert, dass das von der Ultraschal lsonde empfangene RF-signal, das alle ursprüngliche information vor etwaiger Signallbearbeiltunng beinhaltet, für eine externe Datenverarbeitung zur Verfügung stand. Dieses RFsignal wurde einem externen Computer zugeführt, wie auch das. Signal der internen Uhr des Dopplergerătes und der sogenannte Emissionstrigger, der bestimmt, wann ein UItraschallipuls die Sonde verlässt. Dieses auf der Basis der RF-signale basiepte System erreichte eine räumliche Auflösung von ca. $2 \mathrm{~mm}$, die Amplitude des RF -signals wurde als Funktion von Zeit und Tiefe, d.h. Entfernung von der Ultraschallsonde, farbkodiert wiedergegeben. Die Variabilität der MES war erstaunllich gering, so sahen wir einen auffallend gleichbleibenden Tiefebereich, in dem die MES vorkamen, die mit konventionellen Systemen wahrgenommen Veränderungen der Geschwindigkeit konnten nicht bestätigt werden. Wir zagen hieraus dien Schluss, dass die relativ grossen Messvolumina konventioneller Dopplersysteme verantwortlich sind für die eirhebliche Variabilität der Erscheinungsworm von MES, welche somit ein messtechnisch bedingter Artefaktt ist.

\section{Kapite: 6}

Bel etwa 30\% der gasförmigen MES konnte bei der Anwendung der RF-signal Analyse eine Änderung der Flussrichtung im Verhältnis zur Ultraschallsonde festgestellt werden, wobei diese Umkehr nur in wenigen und stets gleichbleibenden Tiefen auftrat. In diesem Kapitel werden die 
Ergebnisse einer Untersuchung worgestellt, die die genannten Umkehipunkte der MES mit der gefässanatomischen Situation verglich, wobei letztere mit der transkraniallen farbkodierten Duplexsonografie beurteilt wurde.

Wir dokumentierten die Lokalisation der Aufzweigung der Arteria carotis interna in die Arteria cerebri anterior und die ACM sowie etwaige sichtbare Seitenäste der ACM bei fünf Patienten präoperativ vor einer Operation am offenen Herzen. Diese Gefässstrukturen zeichnen sich u.a. dadurch aus, dass es zumindest auf einer kurzen strecke zu einer Veränderung der Blutflussrichtung kommt. Während der Operation wurden MES registriert, postoperativ wurde die Tiefe einer eventuellen Flussrichtungsumkehr der MES visuell bestimmt und mit der Gefässanatomie des Patienten auf der Basis der transkraniellen farbkodierten Duplexsonografie verglichen. Es konnte gezeigt werden, dass die Tiefe der Flussumkehir eines MES mit einer Genauigkeit von ca. $2 \mathrm{~mm}$ übereinstimmte mit der Lokalisation einer Gefässgabelung, die wiederum kompatibel ist mit einer Änderung der Strömungsrichtung. Diese Untersuchungsergebnisse demonstrierten, dass es unter Zuhilfenahme der RF-signal Analyse möglich ist, den Verlauf von MES imit einer sehr hohen räumlïchen Auflösung zu verfolgen.

\section{Kapitell 7}

Epilog

In diesem Kapitel werden die Resultate aller Studien im Zusammenhang besprochen. Darüberhinaus wird die Bedeutung der jüngsten Entwicklungen auf dem Gebiet der automatischen Mikroembolidetektion auch im Hinblick auf Zukunftsperspektiven diskutiert. Während die Studien dieser Promotion durchgeführt wurden, sind mehrere signalanallytische Ans.ätze auf der Basis eines Messvolumens vorgeschlagen worden, u.a. eine Frequenzfiltertechnik und ein komplexes "rule-based expert system", das auch Aspekte neuronaler Netzwerke integriert. Daneben wurden Modifikationen der dual-gate Technik worgestellt, wobei eilne Kombination mit der Wavelet Analyse und die Anwendung eines sogenannter willkürlichen Messwolumens hervorzuheben sind. Bei der letztgenannten Technik wird ein Messvolumen absichtlich ausserhalb der grossen intrakraniellen Blutgefässe plaziert, so dass dessen Signal als Referenz dient. Ein weiteres System basiert auf der glleichzeitigen Amwendung zweier Frequenzen und macht sich damit die spezifischen Uitraschalleigenschaften des Mikroembolus im Hinblick auf die ausgesendeten Frequenzen zu Nutzen.

Schlussendlich werden in diesem Kapitel mögliche zukünftige Perspektiven umrissen. Der menschliche Goldstandard für die Detektion won Mikroemboli kann hinterfragt werden. Möglicherweise kann die RF-signal Analyse einen Beitrag zur unzweideutigen Identifikation eines Mikroembolus leisten, indem auch bei kleinen MES, d.h. solchen mit niedriger Amplitude, das Bewegungselement festgestellt wird, welches den Mikroembollus won allen anderen Signalen unterscheidet. Es ist offensichtlich, dass automatische Systeme für die Embolicletektion auf die jeweilige Quelle und somit die Art des embolischen Materials abgestimmt werden müssen und dass die Qualïtät der Systeme im Hinblick auf Sensitivität und Spezifităt durch eine unabhängige 
Untersuchung beurteillt werden muss. Die Mikroembolidetektion wăhrend Karotisendarteriektomie hat sich mittlerweile als klinische Methode etabliert, eine Herausforderung für die Zukunfe dahingegen ist die Identifikation risikoreicher atherosklerotischer Plaques in der Arteria carotis.

Kapitel 8

Appendix

Die Grundlagen won Dopplersystemen werden in diesem Kapitel erläutert, wobei zunächst das Prinzip des kontinuierlichen Dopplers umrissen wird und danach eine allgemeine Beschreibung von gepulsten und intrakraniellen Dopplersystem folgt. Insbesondere wird dabei auf die ráumlichen Eigenschaften des Messwolumens eingegangen.

\section{Schlussfolgerung}

Abschliessend kann gesagt werden, dass die hier worgestellte Arbeit die Komplexität der Beschireibung won MES und damit inhärent auch der Detektion dieser Signale widerspiegelt. Die Interaktion zwischen dem ursprünglichen Ultraschallsignal und seiner weiteren Verarbeitung in konventionellen Dopplersystemen führt zwangsläufig zu falschen Annahmen im Hinblick auf die Eigenschaften won MES. Dadurch wird auch die erfiolgreiche Anwendung der dual-gate Technik, deren Ziel es ist, durch den Einsatz zweier Messvolumina die Bewegung eines MES zu dokumentieren, behindert. Die Analyse des RF.signals dahingeglen macht die tatsächliche Erscheinungsform des MES sichtbar, da die charakteristischen Intensitätsveränderungen in Zeit und Ort, welche ein überraschend gleichbleibendes Muster zeigten, durch diese Methode eindeutig messbar werden.. 


\section{Dankwoord}

Dagmar, Karoline en Max. Zonder jullie was het er niet wan gekomen. En: sorry voor de datum, Karoline.

Mijn ouders hebben mij alle vrijheid gegeven. Dat was niet vanzelfsprekend. Ik draag dit boek aan hun op.

Arnold, het was maar een kwestie van tijd, dat ik bij jou zou gaan aankloppen met de vraag of jij iets willde doen met embolieën. En üneens stond een gemodificeerde Dopplermachine op de OK en moest ik iets doen met Mattab. Ik vondt het prachtig om zo te werken. De helderheid en de snelheid wan jouw commentaar op manuscripten is verbazingwekkend, en bovendien kun jij heel goed inschatten, wanneer aan welke duimschroef gedraaid moet worden. lk kan alleen maar zeggen, dat ik iedere promovendus een zulke promotor als jou toewens.

Rob, jij hebt mij naar "Holland" gehaald en mij aangezet om aan dit proefschrift te beginnen. Het afronden liet op zich wachten, maar nu is de kogel door de kerk en dat heb ik vooral ook aan jou te danken. Jij was altijd duidelijk, dat maakte het werken in het Antonius in thet algemeen en aan dit proefschrift in het bijzonder effectief. Jij was streng, maar had altijd waardering en respect. En wie anders hangt er nou een historische kaart van Duitsland op de gang, om in een pauze eens naar bijzondere plaatsen te kijken?

David, maybe untill the $2^{\text {nd }}$ of May, you did not know exactly, what a Dutch thesis would mean. Nevertheless, you never let me in doubt, that you would support me. Working with you was inspiring. Possibly, you remember we discussed the sensitivity of the sample walume sitting on at stony step in an old palace in venice...

Bart, toen jij kwam, heb jij heel wat heilige emboli-huisjes omver gestoten. En gelijk had jij ook nog. Maar je bent wooral ook een aardig mens, de tijd in Nieuwegein met z'n drieën op enkele vierkante meters was een mooie tijd.

Jean, jouw jeugdig enthousiasme werkte op mij aanstekelijk, en vooral: lk kon je alltijd van alles vragen, ook at was het woor de zoveelste keer. Als ik dan lets had begrepen, was jij misschien met zo blij als ik.

Dit proefschrift kan niet los worden glezien wan het feit, dat ik bijna 20 jalar geleden de eerste stappen op het gebied van ultrageluid en Doppler metingen deed. Mwr. Lippe leerde mij toen hoe ik met de Delalande moest werken, later liet mwr. Hache mij zien hoe de transcraniële Doppler functioneerde. Dit alles gebeurde onder supervisie van dr. Rautenberg en dr. Steinke. Door hun uitleg en geduld kon ik de nodige ervaring op ultrageluidsgebied opbouwen. 
Mijn collega's uit de tijd in Mannheim mogen evenmin onopgemerkt blijven. Michael, Rolf, Ulrich, Joachim, Christof, Hans, Thomas en anderen: het waren drukke tijden resulterend in woldoende verhaten om aan kleinkinderen te vertellen. Wij hebben veel van elkaar geleerd, waarwoor dank.

Prof. Hennerici, Sie haben mich als studentische Hilfskraft angenommen und später als Assistent. Ohne Sie stände ich heute nicht da, wo ich bin. Niemand hat einen grösseren Einfluss auff mein Studium und meine Ausbildung gehabt als Sie. Als ich Sie anrief mit der Frage, in der Beurteillungskommission dieser Promotion mitzuarbeiten, zögerten Sie keinen Augemblick. Auch dafür mö́chte ich mich bei Ihnen bedanken.

Essentieel was ook de enthousiaste medewerking van de laboranten wan de afdeling KNF van het Antonius Ziekenhuis, vooral natuurlijk Marianne van der Mee. Maar ook vele andlere medewerkers vam het Antonius, in het bijzonder Frans Moll, Freddie Vermeulen, later Marc Schepens en natuurijik Chuck van de Vlasakker, hebben voor milj de beslissing makkelijk gemaakt on aan deze kant van de Nederlands-Duitse grens te werken en ook te promoveren.

Mijn dank gaat ook naar vele mensen in het azM, die mij op uiteenlopende manieren bij dit proefschrift ondersteund thebben. Er was praktische ondersteuning van de medewerkers van de afdeling KNF en ik kreeg voldoende ruimte om de metingen en de data analyse te verrichten alsmede de nodige stukken te schrijven. Op de OK was er altijd volop hulp, ook al was het soms niet zo duidelijk wat er precies gebeurde. En:zonder" coöperatieve chirurgen geen embolie detectie op de OK!

Rest mij nog Jan Meinders en Jeroen Hameleers te bedanken voor hun praktische medewerking in de slotfase. Ook was de sfeer op jullie lab woor mij bellangrijk.

Tensllotte wil ik Fabrice Hermans bedanken. Uw manier van werken is woor mij een voarbeeld. 


\section{Publications}

Rautitenberg W, Hennerici M, 5 chär D, Mess W. Spontanverlauf und konservative Therapie extrakranieller Karotisprozesse. In: Aktuelle Diagnostik und Therapie in der Angiologie. (Ed: Kriessmann,A) Georg Thieme Verlag, 1988, pp. 90-94.

Rautenberg W. Mess W. Henneric: M. Prognosis of asymptomatic canotid acclusion. J Neurol Sci 1990;98:213-220.

Hennerici $M_{4}$ Mess W, Rautenberg W. Zerebrale Durchblutungsstörungen - Klinik und Verlauf. Acta Med Austriaca 1991;18:34-38.

Rautenberg $W_{i}$ Mess $W_{s}$ Hennericil M. Asymptomatische Karotisprozesse - welche Formen sollen operativ behandelt werden? Vasa 1992;35(suppl): 9-10.

Pohlmann-Eden B, Wolf T, Mess W, DiehI RR. Psychogene Blindheit. Akt Neurol 1993;20:58-61.

Mess W, Klimke A, Klieser E. Untersuchungen mit der transkraniellen Dopplersonografie bei Elektrokrampfbehandlung. In: Aktuelle Perspektiven der Biologischen Psychiatrie. (Eds: Möller HJ, Müller-Spahn G، Kurtz G) Wien: Springer, 1996, pp.212-216.

Mess W, Klimke A, Saimeh N, Klieser E. (1996): Fallbericht: Paradoxe Hirnembolie als Komplikation der Elektrokrampfbehandlung. In: Aktuelle Perspektiven der Biologischen Psychiatrie.

(Eds: Möller HJ, Müller-Spahn G, Kurtz G) Wien:Springer 1996, pp.244-246.

van Zuilen EV, Mess WH, Jansen C, van der Tweel I, van Gijn J, Ackerstaff RGA. Automatic embolus detection compared with human experts; a Doppler ultrasound study. Stroke 1996;27:1840-1843.

Ackerstaff RGA, Mess WH, van Zuilen EV, van der Tweel I. Reliability in the identification of Doppler embolic transient signals: a statistical problem? (Letter) Stroke 1997;28:876.

Mess WH, Lodder J. Air emboli and prosthetic heart valves. (Letten) Stroke 1997;28:2570-2571.

Mess WH, Titulaer BM, Ackerstaff RGA. Discrimination and cliaracterization of emboli: old and new aspects. In: New Trends in Cerebral Hemodynamics and Neurosonology. (Eds: Klingelhöfer J, Bartels E Ringelstein EB) Elsevier, Amsterdam 1997,355-363. 
Keunen RWM, Stam CJ, Tawy DU, Mess WH, Titulaer BM, Ackerstaff RGA. Preliminary report of detecting microembolic signals in transcranial Doppler time series with nonlinear forecasting. Stroke 1998:29:1638-1643.

Claus SP, Louwerse ES, Mauser HW, van der Mee M, Moll FL, Mess WH, Ackerstaff RGA. Temporary occlusion of middle cerebral artery by macroembolism in carotid surgery.

Cerebrowasc Dis 1999;9:261-264.

Mess WH, Titulaer BM, Ackerstaff RGA. Middle cerebral artery anatomy and characteristics of embolic signals - a dual gate computer simulation study. U/trasound Med Bial 1999;25:531-539.

Backes WH, Mess WH, van Kranen-Mastenbroek VHעM, Reulen JPH. Somatosensory cortex responses to median nerve stimulation: MMRI effects of current amplitude and selective attention.

Clin Neurophysiol 2000;111:1738-1744.

Mess WH, Titulaer BM, Ackerstaff RGA. A new algorithm for off-line automated emboli detection based on the pseudo-Wiginer power distribution and the duall gate TCD technique.

Ultrasound Med Biol 2000;26:413-418.

Backes WH, Mess WH, Wilmink JT. Functional MR responses of the cervical spinal cord upon sensory stimulation and motor tasks. Neuroradiology 2000:42 (suppl 1):530-531.

Hoving MA; Mess WH, Weber WEJ. Arteriitis temporalis: kleuren-duplex in plaats van biopsie? Ned Tijdschrift Neurologie 2001;4:209-214.

Backes WH, Mess 'WH, Wilmink JT. Functional MR imaging of the cervical spinal cond using median nerve stimulation and fist clenching. ANNR 2001;22:1854-1859.

Mess WH, Hennerici MG. High Intensity Transient Signalsi In: Cerebrovascular Ultrasound; Theory, Practice and Future Developments. (Eds: Hennerici M, Meairs S) Cambridge University Press, Cambridge 2001, 297-316.

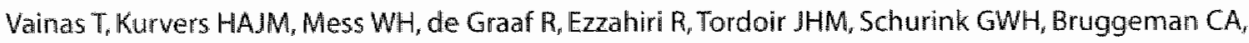
Kitslaar PJEHM. Chlamydia pneumoniae (Cpn) serology is associated with thrombosis-related but not with plaque-related micro-embolization during carotid endarterectomy. Stroke 2002;33:1249. 1254.

Jacobs MJ, de Mol BA, Elenbaas T, Mess WH, Kalkman CJ, Schurink GW, Mochtar B. Spinal cord blood supply in patients with thoracoabdominal aortic aneurysms. I Vasc Surg 2002;35:30-37. 
Jacobs MJ, Elenbaas TW. Schurink GWH, Mess WH, Mochtar B. Assessment of spinal cord integrity during thoracoabdominal aortic aneurysm repair. Ann Thorac 5urg 2002;74:51864-51866.

Mess WH, Willigers JM, Ledoux LAF, Ackerstaff RGA, Hoeks APG. Microembolic signal description: A reappraisal based on a customized digital postprocessing system. Ultrasound Med Biol 2002 28:1415-1423.

Munts $A G$, Mess WH, Bruggemans. EF, Walda LA, Ackerstaff RGA. Feasibility and relability of on-line automated microemboli detection after carotid endarterectomy. A transcranial Doppler study. Eur I Vasc Endovasc Surg 2003;25:262-266. 


\section{Curriculum Vitce Werner Mess werd geboren op 7 februari 1961 te Recke in Westfalen}

(Duitsland) als eerste kind van Ewald en Margret Mess. Na het Abitur in 1980 te hebben behaald, volgden de vervangende dienst in een bejaardenhuis in Stuttgart en een jaar aan de Universiteit Münster met als vakken Frans en geschiedenis. In 1981 begon hij de studie Geneeskunde aan de Universiteit Düsseldorf en in november 1989 behaalde hij het artsexamen. In januari 1990 startte de opleiding tot neuroloog, waarbij het neurologisch gedeelte in Manmheim, en vanaf mei 1993 het psychiatrisch gedeelte in Düsseldorf plats vonden. Hij legde het specialistenexamen neurologie in december 1994 af, tevens promoveerde hij in dezelfde maand in Heidelberg (Inauguraldissertation) ower het spontane beloop van patienten met een ernstige vernauwing van de halsslagaders. In januari $1995 \mathrm{kwam}$ hij nalar Nederland en werkte tot februari 1997 als chef de laboratoire op de afdeling Klinische Neurofysiologie van het St. Antonius Ziekenhuis, Nieuwegein. In maart 1997 werd hij ingeschreven in het Nederlandlse specialistenregister als neuroloog met aantekening klinische neurofysiologie en sindsdien werkt hij als klinisch neurofysioloog in het Academisch Ziekenhuis Maastricht.

Hij is sinds 1993 getrouwd met Dagmar, zijn dochter heet Karoline en zijn zoon Max. 
00555 

